\title{
GAUGE THEORY ON INFINITE CONNECTED SUM AND MEAN DIMENSION
}

\author{
MASAKI TSUKAMOTO
}

\begin{abstract}
We study the geometry of infinite dimensional moduli spaces coming from the Yang-Mills gauge theory over infinite connected sum spaces. We develop the technique of gluing infinitely many instantons, and apply it to the evaluation of the mean dimension of the moduli spaces.
\end{abstract}

\section{INTRODUCTION}

Nonlinear analysis on non-compact manifolds is a challenging research field. We study the infinite energy Yang-Mills gauge theory on certain non-compact 4-manifolds (infinite connected sums of $\left.S^{4}\right)$.

Let $\Gamma$ be a finitely generated infinite group with a finite generating set $S$. We suppose that $S$ does not contain the identity element. We consider the infinite connected sum space $\left(S^{4}\right)^{\sharp(\Gamma, S)}$ by gluing the copies of $S^{4}$ "along the Cayley graph of $(\Gamma, S)$ ". (Its precise definition will be given in Section 2.) $\left(S^{4}\right)^{\sharp(\Gamma, S)}$ is a non-compact 4-manifold. $\Gamma$ naturally acts on $\left(S^{4}\right)^{\sharp(\Gamma, S)}$. For example, if $(\Gamma, S)=(\mathbb{Z},\{1\})$, then $\left(S^{4}\right)^{\sharp(\Gamma, S)}$ is conformally equivalent to $S^{3} \times \mathbb{R}$.

Fix $c>0$. We want to study $S U(2)$-ASD connections $A$ on $\left(S^{4}\right)^{\sharp(\Gamma, S)}$ satisfying $\left\|F_{A}\right\|_{L^{\infty}} \leq c$. (Here we consider " $L^{\infty}$-norm condition" for simplicity of the explanation. We will consider more general conditions later.) Since the base space $\left(S^{4}\right)^{\sharp(\Gamma, S)}$ is non-compact, such ASD connections can have infinite $L^{2}$-energy, and their moduli space $\mathcal{M}$ can be an infinite dimensional space. The moduli space $\mathcal{M}$ admits a natural $\Gamma$-action. The main subject of this paper is the evaluation of the "mean $\operatorname{dimension"} \operatorname{dim}(\mathcal{M}: \Gamma)$. Mean dimension is a notion introduced by Gromov [8]. (See also Lindenstrauss-Weiss [10] and Lindenstrauss [9].) Intuitively, the mean $\operatorname{dimension} \operatorname{dim}(\mathcal{M}: \Gamma)$ is given by

$$
\operatorname{dim}(\mathcal{M}: \Gamma)=\operatorname{dim} \mathcal{M} /|\Gamma|
$$

Date: February 4, 2009.

Key words and phrases. Yang-Mills gauge theory, mean dimension, infinite connected sum, gluing infinitely many instantons.

This work was supported by Grant-in-Aid for JSPS Fellows (19.1530) from Japan Society for the Promotion of Science. 
(We give the precise definition of mean dimension in Appendix B.) In particular, if $\mathcal{M}$ is a finite dimensional space (in the usual sense), then $\operatorname{dim}(\mathcal{M}: \Gamma)=0$. Hence the value of $\operatorname{dim}(\mathcal{M}: \Gamma)$ has an information about "infinite dimensional geometry" of $\mathcal{M}$.

We study $\mathcal{M}$ by using the technique of "gluing an infinite number of instantons". Gluing instantons is a famous technique in the gauge theory. (Taubes [12], Donaldson [3], etc.) In Tsukamoto [13], we studied the technique of gluing infinitely many instantons. In the present paper we will develop this gluing technique more thoroughly and apply it to the evaluation of the mean $\operatorname{dimension} \operatorname{dim}(\mathcal{M}: \Gamma)$. The main body of the paper is devoted to the detailed (rather technical) study of this infinite gluing construction.

The application of the gluing technique to the theory of mean dimension is suggested by Gromov [8, p. 403, 3.6.6] in the context of (pseudo-)holomorphic curves. Gournay [7] studies the application of the gluing technique of pseudo-holomorphic curves to the problem of mean dimension.

\section{Gauge theory on infinite CONneCted Sum of $S^{4}$}

2.1. Main results. Let $\Gamma$ be a finitely generated infinite group. Let $S=\left\{s_{1}, \cdots, s_{|S|}\right\} \subset$ $\Gamma$ be a finite generating set which does not contain the identity element. Here we don't suppose that $S=S^{-1}$. Easy examples are $(\Gamma, S)=(\mathbb{Z},\{1\}),\left(\mathbb{Z}^{2},\{(1,0),(0,1)\}\right)$.

Let $S^{4}$ be the 4 -sphere and $x_{s}$ and $y_{s}(s \in S)$ be $2|S|$ distinct points in $S^{4}$. We will construct an infinite connected sum $\left(S^{4}\right)^{\sharp(\Gamma, S)}$ by patching the copies of $S^{4}$ "along the Cayley graph of $(\Gamma, S)$ ". The following construction is based on the "conformal connected sum" described in Donaldson-Kronheimer [5, Section 7.2].

Since the standard metric on $S^{4}$ is conformally flat, $S^{4}$ has a Riemannian metric $h$ satisfying the following:

(i) $h$ is conformally equivalent to the standard metric.

(ii) $h$ is flat in some neighborhood of each $x_{s}$ and $y_{s}(s \in S)$.

Of course, $h$ is not uniquely determined by these conditions. The important condition is the first one. The second condition is just for simplicity. Let $\lambda$ be a positive (very small) parameters. For $x \in S^{4}$ and $r>0$, we denote $B(x, r)(\operatorname{resp} . \bar{B}(x, r))$ as the open (resp. closed) ball of radius $r$ centered at $x$ (with respect to the metric $h$ ). Set

$$
U:=S^{4} \backslash\left(\bigsqcup_{s \in S} \bar{B}\left(x_{s}, \sqrt{\lambda} / 2\right) \sqcup \bar{B}\left(y_{s}, \sqrt{\lambda} / 2\right)\right) .
$$

For each $s \in S$ we choose an orientation-reversing linear isometry $\sigma_{s}: T_{x_{s}} S^{4} \rightarrow T_{y_{s}} S^{4}$.

For each $\gamma \in \Gamma$, let $\left(S_{\gamma}^{4}, h_{\gamma}\right)$ be the isometric copy of $\left(S^{4}, h\right)$. Let $x_{\gamma, s}$ and $y_{\gamma, s}(s \in S)$ be the points on $S_{\gamma}^{4}$ corresponding to $x_{s}$ and $y_{s}$ on $S^{4}$. $S_{\gamma}^{4}$ has the open set $U_{\gamma}$ corresponding to $U$ in $S^{4}$. We define the connected sum $\left(S^{4}\right)^{\sharp(\Gamma, S)}$ by

$$
\left(S^{4}\right)^{\sharp(\Gamma, S)}:=\left(\bigsqcup_{\gamma \in \Gamma} U_{\gamma}\right) / \sim,
$$


where the identification $\sim$ is given as follows; We identify the annulus region $B\left(x_{\gamma, s}, 2 \sqrt{\lambda}\right) \backslash$ $\bar{B}\left(x_{\gamma, s}, \sqrt{\lambda} / 2\right)$ in $S_{\gamma}^{4}$ with $B\left(y_{\gamma s, s}, 2 \sqrt{\lambda}\right) \backslash \bar{B}\left(y_{\gamma s, s}, \sqrt{\lambda} / 2\right)$ in $S_{\gamma s}^{4}$ by

$$
\begin{aligned}
B\left(x_{\gamma, s}, 2 \sqrt{\lambda}\right) \backslash \bar{B}\left(x_{\gamma, s}, \sqrt{\lambda} / 2\right) & \ni \xi \sim \eta \in B\left(y_{\gamma s, s}, 2 \sqrt{\lambda}\right) \backslash \bar{B}\left(y_{\gamma s, s}, \sqrt{\lambda} / 2\right), \\
& \Longleftrightarrow \eta=\lambda \sigma_{s}(\xi) /|\xi|^{2} .
\end{aligned}
$$

Here $\xi$ and $\eta$ are the normal coordinates centered at $x_{\gamma, s}$ and $y_{\gamma s, s}$, and we consider $\sigma_{s}$ as a map from $T_{x_{\gamma, s}} S_{\gamma}^{4}$ to $T_{y_{\gamma s, s}} S_{\gamma s}^{4}$ by identifying $T_{x_{\gamma, s}} S_{\gamma}^{4}$ (resp. $T_{y_{\gamma s, s}} S_{\gamma s}^{4}$ ) with $T_{x_{s}} S^{4}$ (resp. $\left.T_{y_{s}} S^{4}\right) .\left(S^{4}\right)^{\sharp(\Gamma, S)}$ admits a natural left $\Gamma$-action as follows. For $\delta \in \Gamma$ we define $\delta: U_{\gamma} \rightarrow U_{\delta \gamma}$ by sending $p \in U_{\gamma}$ to $q \in U_{\delta \gamma}$ corresponding to the same point in $S^{4}$. This is compatible with the above identification (1). This action is fixed point free, i.e., every $\delta \neq 1$ in $\Gamma$ has no fixed point.

We choose a $\Gamma$-invariant Riemannian metric $g$ on $\left(S^{4}\right)^{\sharp(\Gamma, S)}$ as follows; Let $w$ be a smooth function in $S^{4}$ such that $0 \leq w \leq 1$ all over $S^{4}, w=1$ in the complement of the balls $B\left(x_{s}, \sqrt{\lambda}\right)$ and $B\left(y_{s}, \sqrt{\lambda}\right)(s \in S)$, and $w=0$ on each $\bar{B}\left(x_{s}, \sqrt{\lambda} / 2\right)$ and $\bar{B}\left(y_{s}, \sqrt{\lambda} / 2\right)$. Let $w_{\gamma}(\gamma \in \Gamma)$ be the copy of $w$ defined in $S_{\gamma}^{4}$. We set

$$
g:=\sum_{\gamma \in \Gamma} w_{\gamma} h_{\gamma}
$$

where $h_{\gamma}$ is the Riemannian metric given before. Since the map $\eta=\lambda \sigma_{s}(\xi) /|\xi|^{2}$ in (1) is conformal, $g$ is conformally equivalent to each $h_{\gamma}$ over $U_{\gamma}$.

We want to study $S U(2)$-ASD connections over $\left(S^{4}\right)^{\sharp(\Gamma, S)}$. Let $c>0$ be a positive real number and $d \in(2,+\infty](d$ may be $+\infty)$. Let $E$ be a principal $S U(2)$-bundle over $\left(S^{4}\right)^{\sharp(\Gamma, S)}$ and $A$ be an ASD connection on $E$. We want to study such a pair $(E, A)$ satisfying

$$
\left\|F_{A}\right\|_{L^{d}\left(U_{\gamma}, g\right)} \leq c \text { for all } \gamma \in \Gamma,
$$

where the norm denotes the $L^{d}$-norm over the region $U_{\gamma}$ defined by the metric $g$. Let $(E, A)$ and $\left(E^{\prime}, A^{\prime}\right)$ be two pairs of a principal $S U(2)$-bundle over $\left(S^{4}\right)^{\sharp(\Gamma, S)}$ and an ASD connection on it. They are called gauge equivalent if there exists a bundle isomorphism $u: E \rightarrow E^{\prime}$ satisfying $u(A)=A^{\prime}$. We define $\mathcal{M}(c, d)$ as the space of the gauge equivalence classes $[E, A]$ of a principal $S U(2)$-bundle $E$ over $\left(S^{4}\right)^{\sharp(\Gamma, S)}$ and an ASD connection $A$ on $E$ satisfying (2). This space admits a natural right $\Gamma$-action: For $[E, A] \in \mathcal{M}(c, d)$ and $\gamma \in \Gamma$, we set

$$
[E, A] \cdot \gamma:=\left[\gamma^{*} E, \gamma^{*} A\right]
$$

where $\gamma^{*} E$ and $\gamma^{*} A$ are the pull-backs of $E$ and $A$ by the map $\gamma:\left(S^{4}\right)^{\sharp(\Gamma, S)} \rightarrow\left(S^{4}\right)^{\sharp(\Gamma, S)}$.

Remark 2.1. Since $\left(S^{4}\right)^{\sharp(\Gamma, S)}$ is a non-compact 4-manifold, all principal $S U(2)$-bundle on it is isomorphic to the product bundle $\left(S^{4}\right)^{\sharp(\Gamma, S)} \times S U(2)$. Therefore we can define $\mathcal{M}(c, d)$ as the space of gauge equivalence classes of ASD connections on $\left(S^{4}\right)^{\sharp(\Gamma, S)} \times S U(2)$ satisfying (2). But the above formulation is more flexible. 
Remark 2.2. An ASD connection $A$ satisfying the condition (2) is a Yang-Mills analogue of "Brody curve" in the theory of entire holomorphic curves. (cf. Brody [2], Tsukamoto $[14,15]$.) A holomorphic curve $f: \mathbb{C} \rightarrow \mathbb{C} P^{N}$ is called a Brody curve if it satisfies $|d f|(z) \leq 1$ (or $|d f|(z) \leq C$ for some positive constant $C$ ) for all $z \in \mathbb{C}$.

$\mathcal{M}(c, d)$ is equipped with the topology of $\mathcal{C}^{\infty}$-convergence on compact subsets. That is, a sequence $\left\{\left[E_{n}, A_{n}\right]\right\}_{n \geq 1} \subset \mathcal{M}(c, d)$ converges to $[E, A] \in \mathcal{M}(c, d)$ if for any compact set $K \subset\left(S^{4}\right)^{\sharp(\Gamma, S)}$ there exist $n_{0}(K)>0$ and bundle maps $u_{n}:\left.\left.E_{n}\right|_{K} \rightarrow E\right|_{K}$ (for $n \geq n_{0}(K)$ ) such that $u_{n}\left(\left.A_{n}\right|_{K}\right)$ converge to $\left.A\right|_{K}$ in the $\mathcal{C}^{\infty}$-topology. This topology is metrizable.

From $d>2$ and Uhlenbeck's compactness result ([16, Theorem 1.5 (3.6)], [17]), the moduli space $\mathcal{M}(c, d)$ becomes compact. But I think that this compactness is not so obvious. In some cases (e.g. $(\Gamma, S)=(\mathbb{Z},\{1\}))$ it directly follows from [17, Theorem E']. But the general case needs some clarification. So we will give its proof in Appendix A.

The group action $\mathcal{M}(c, d) \times \Gamma \rightarrow \mathcal{M}(c, d)$ defined in (3) is continuous. If $\Gamma$ is amenable, then we can define the mean $\operatorname{dimension} \operatorname{dim}(\mathcal{M}(c, d): \Gamma)$. (See Gromov [8], LindenstraussWeiss [10], Lindenstrauss [9] and Appendix B.)

For $c>0$ and $d>2$ we define $M_{S^{4}}(c, d)$ as the space of the gauge equivalence classes of $S U(2)$-ASD connections $[A]$ on $S^{4}$ satisfying

$$
\left\|F_{A}\right\|_{L^{d}\left(S^{4}, h\right)} \leq c
$$

We denote $\operatorname{dim} M_{S^{4}}(c, d)$ as the topological (covering) dimension of $M_{S^{4}}(c, d)$. For $d \in$ $(2,+\infty](d$ may be $+\infty)$, we set

$$
\begin{aligned}
c_{0}(d) & :=\sup \left\{c \geq 0 \mid \operatorname{dim} M_{S^{4}}(c, d)=0\right\}, \\
& =\sup \left\{c \geq 0 \mid M_{S^{4}}(c, d)=\{[\text { the product connection] }\}\} .\right.
\end{aligned}
$$

We have $c_{0}(d)>0$, and $\operatorname{dim} M_{S^{4}}(c, d)>0$ for any $c>c_{0}(d)$. Our main results on the gauge theory over $\left(S^{4}\right)^{\sharp(\Gamma, S)}$ are the following. The first result concerns with the upper bound on the mean dimension:

Theorem 2.3. (i) For any $d \in(2,+\infty]$ and $0 \leq c<c_{0}(d)$ there exists $\lambda_{0}(c, d)>0$ such that if $\lambda \leq \lambda_{0}(c, d)$ then $\mathcal{M}(c, d)$ is equal to the space of the gauge equivalence classes of flat $S U(2)$-connections on $\left(S^{4}\right)^{\sharp(\Gamma, S)}$. Hence if $\left(S^{4}\right)^{\sharp(\Gamma, S)}$ is simply connected, then $\mathcal{M}(c, d)$ is the one-point space.

(ii) Suppose $\Gamma$ is amenable. Then for any $0 \leq c<\bar{c}<+\infty$ and $d \in(2,+\infty]$, there exists $\lambda_{1}(c, \bar{c}, d)>0$ such that if $\lambda \leq \lambda_{1}(c, \bar{c}, d)$ then

$$
\operatorname{dim}(\mathcal{M}(c, d): \Gamma) \leq 3|S|+\operatorname{dim} M_{S^{4}}(\bar{c}, d) .
$$

The next result is the lower bound on the mean dimension.

Theorem 2.4. Suppose $\Gamma$ is amenable. Let $0<\underline{c}<c<+\infty$ and $2<d<+\infty$ (d must be finite). If $\operatorname{dim} M_{S^{4}}(\underline{c}, d)>0$, then there exists $\lambda_{2}(\underline{c}, c, d)>0$ such that for any 
$\lambda \leq \lambda_{2}(\underline{c}, c, d)$

$$
\operatorname{dim}(\mathcal{M}(c, d): \Gamma) \geq 3|S|+\operatorname{dim} M_{S^{4}}(\underline{c}, d) .
$$

Theorem 2.5. Suppose $\Gamma$ is amenable. For each $d \in(2,+\infty)$ there exists a countable set $\Delta(d) \subset\left(c_{0}(d),+\infty\right)$ satisfying the following: For any $c \in\left(c_{0}(d),+\infty\right) \backslash \Delta(d)$ there exists $\lambda_{3}(c, d)>0$ such that if $\lambda \leq \lambda_{3}(c, d)$ then we have

$$
\operatorname{dim}(\mathcal{M}(c, d): \Gamma)=3|S|+\operatorname{dim} M_{S^{4}}(c, d) .
$$

We will prove Theorem 2.3 and 2.4 in Section 8 and 9. Here we prove Theorem 2.5, assuming Theorem 2.3 and 2.4 .

Proof of Theorem 2.5. Consider the following non-decreasing function

$$
\left(c_{0}(d),+\infty\right) \rightarrow \mathbb{Z}_{>0}, \quad c \mapsto \operatorname{dim} M_{S^{4}}(c, d)
$$

Let $\Delta(d) \subset\left(c_{0}(d),+\infty\right)$ be the set of points where (4) is not continuous. $\Delta(d)$ is a countable set. For any $c \in\left(c_{0}(d),+\infty\right) \backslash \Delta(d)$, if we choose $\underline{c}$ and $\bar{c}(\underline{c}<c<\bar{c})$ sufficiently close to $c$, then we have

$$
\operatorname{dim} M_{S^{4}}(\underline{c}, d)=\operatorname{dim} M_{S^{4}}(c, d)=\operatorname{dim} M_{S^{4}}(\bar{c}, d)>0 .
$$

Using Theorem 2.3 and 2.4, we get

$$
\operatorname{dim}(\mathcal{M}(c, d): \Gamma)=3|S|+\operatorname{dim} M_{S^{4}}(c, d),
$$

for $\lambda \ll 1$.

2.2. Outline of the proofs of the main theorems. The proofs of the main theorems (Theorem 2.3, 2.4) need lengthy technical arguments. So we want to describe the brief outline of the proofs in this subsection.

For $c \geq 0$ and $d \in(2,+\infty]$, we call $\theta=\left(E_{\gamma}, A_{\gamma}, \rho_{\gamma, s}\right)_{\gamma \in \Gamma, s \in S}$ a $(c, d)$-gluing data if the following conditions are satisfied: Each $E_{\gamma}(\gamma \in \Gamma)$ is a principal $S U(2)$-bundle over $S_{\gamma}^{4}$, and $A_{\gamma}$ is an ASD connection on $E_{\gamma}$ satisfying $\left\|F\left(A_{\gamma}\right)\right\|_{L^{d}\left(S_{\gamma}^{4}, h_{\gamma}\right)} \leq c . \rho_{\gamma, s}$ is an $S U(2)$ isomorphism from $\left(E_{\gamma}\right)_{x_{\gamma, s}}$ to $\left(E_{\gamma s}\right)_{y_{\gamma s, s}}$. We can consider a natural equivalence relation in the set of $(c, d)$-gluing data. For each $(c, d)$-gluing data $\theta$, we will construct a principal $S U(2)$-bundle $\boldsymbol{E}(\theta)$ over $\left(S^{4}\right)^{\sharp(\Gamma, S)}$ and an ASD connection $\boldsymbol{A}(\theta)$ on it by using a "gluing construction".

The proof of Theorem 2.3 proceeds as follows; $0 \leq c<\bar{c}<+\infty$ and $d \in(2,+\infty]$. For each $[E, A] \in \mathcal{M}(c, d)$ we can find a $(\bar{c}, d)$-gluing data $\theta$ satisfying $[E, A]=[\boldsymbol{E}(\theta), \boldsymbol{A}(\theta)]$ $(\lambda \ll 1)$. (This is the most difficult part of the proof.) If $\bar{c}<c_{0}(d)$, then we can (easily) prove that $\boldsymbol{A}(\theta)$ is flat. This shows Theorem 2.3 (i). In general, we have

$$
\operatorname{dim}(\mathcal{M}(c, d): \Gamma) \leq \operatorname{dim}(\text { space of }(\bar{c}, d) \text {-gluing data }: \Gamma),
$$

and the right-hand-side can be estimated as follows. (The following argument is not rigorous.) Let $\theta=\left(E_{\gamma}, A_{\gamma}, \rho_{\gamma, s}\right)_{\gamma \in \Gamma, s \in S}$ be a $(\bar{c}, d)$-gluing data. For each $\gamma \in \Gamma,\left(E_{\gamma}, A_{\gamma}\right)$ 
has $\operatorname{dim} M_{S^{4}}(\bar{c}, d)$ parameters of deformation, and $\rho_{\gamma, s}$ has three parameters (for each $s \in$ $S)$. Therefore the number of "deformation parameters" of $\theta$ is $\left(\operatorname{dim} M_{S^{4}}(\bar{c}, d)+3|S|\right)|\Gamma|$.

Hence we have

$$
\begin{aligned}
\operatorname{dim}(\text { space of }(\bar{c}, d) \text {-gluing data }: \Gamma) & =\operatorname{dim}(\text { space of }(\bar{c}, d) \text {-gluing data }) /|\Gamma|, \\
& \approx \operatorname{dim} M_{S^{4}}(\bar{c}, d)+3|S| .
\end{aligned}
$$

From this we get Theorem 2.3 (ii).

On the other hand, if $0<\underline{c}<c<\infty$ and $d \in(2,+\infty)$ then we can prove that, for each $(\underline{c}, d)$-gluing data $\theta,[\boldsymbol{E}(\theta), \boldsymbol{A}(\theta)]$ belongs to $\mathcal{M}(c, d)$. (The proof of this facts needs $d<\infty$.) Therefore we have

$$
\operatorname{dim}(\mathcal{M}(c, d): \Gamma) \geq \operatorname{dim}(\text { space of }(\underline{c}, d) \text {-gluing data }: \Gamma) .
$$

Using (5), we get Theorem 2.4. The above argument does not explain the meaning of the condition "dim $M_{S^{4}}(\underline{c}, d)>0$ " in the assumption in Theorem 2.4. This condition concerns with the validity of the equation (5).

\section{INFINITE GLUING CONSTRUCTION: PREPARATIONS}

From this section we will develop a theory of "gluing infinitely many instantons" for general closed 4-manifolds. Let $X$ be a compact, oriented Riemannian 4-manifold with prescribed $2|S|$-points $x_{s}$ and $y_{s}(s \in S)$. We suppose that the metric is flat in some neighborhood of each $x_{s}$ and $y_{s}$. Fix a real number $p$ with $2<p<4$ and define $q \in$ $(4,+\infty)$ by $1-4 / p=-4 / q$, i.e., $L_{1}^{p} \hookrightarrow L^{q}$. (These $p$ and $q$ are fixed throughout the paper.)

3.1. Infinite connected sum. First we briefly describe a construction of an infinite connected sum of $X$. This is essentially the same as in Section 2. But we need to introduce one more extra parameter $N>0$ for several technical reasons. Let $\lambda$ and $N$ be positive parameters. We choose them so that $\lambda \ll 1, N \gg 1$ and $N \sqrt{\lambda} \ll 1$. We set (we follow the notation of Donaldson-Kronheimer [5, Section 7.2])

$$
\begin{aligned}
& X^{\prime}:=X \backslash\left(\bigsqcup_{s \in S} \bar{B}\left(x_{s}, \sqrt{\lambda} / N\right) \sqcup \bar{B}\left(y_{s}, \sqrt{\lambda} / N\right)\right), \\
& X^{\prime \prime}:=X \backslash\left(\bigsqcup_{s \in S} \bar{B}\left(x_{s}, \sqrt{\lambda} / 2\right) \sqcup \bar{B}\left(y_{s}, \sqrt{\lambda} / 2\right)\right) .
\end{aligned}
$$

$X^{\prime \prime}$ corresponds to the region $U$ in Section 2. We define annulus regions $\Omega\left(x_{s}\right)$ and $\Omega\left(y_{s}\right)$ in $X$ by $\Omega\left(x_{s}\right):=B\left(x_{s}, N \sqrt{\lambda}\right) \backslash \bar{B}\left(x_{s}, \sqrt{\lambda} / N\right)$ and $\Omega\left(y_{s}\right):=B\left(y_{s}, N \sqrt{\lambda}\right) \backslash \bar{B}\left(y_{s}, \sqrt{\lambda} / N\right)$. For each $s \in S$ we choose an orientation-reversing isometry $\sigma_{s}: T_{x_{s}} X \rightarrow T_{y_{s}} X$.

Let $X_{\gamma}(\gamma \in \Gamma)$ be the copy of $X$ with the points $x_{\gamma, s}$ and $y_{\gamma, s}(s \in S)$ corresponding to $x_{s}$ and $y_{s} . X_{\gamma}$ has the open sets $X_{\gamma}^{\prime}, X_{\gamma}^{\prime \prime}, \Omega\left(x_{\gamma, s}\right), \Omega\left(y_{\gamma, s}\right)$ corresponding to $X^{\prime}, X^{\prime \prime}, \Omega\left(x_{s}\right)$, 
$\Omega\left(y_{s}\right)$ in $X$, respectively. We define $X^{\sharp(\Gamma, S)}$ by

$$
X^{\sharp(\Gamma, S)}:=\left(\bigsqcup_{\gamma \in \Gamma} X_{\gamma}^{\prime}\right) / \sim,
$$

where we identify $\Omega\left(x_{\gamma, s}\right)$ in $X_{\gamma}^{\prime}$ with $\Omega\left(y_{\gamma s, s}\right)$ in $X_{\gamma s}^{\prime}$ by

$$
\Omega\left(x_{\gamma, s}\right) \ni \xi \sim \eta \in \Omega\left(y_{\gamma s, s}\right) \stackrel{\text { def }}{\Longleftrightarrow} \eta=\lambda \sigma_{s}(\xi) /|\xi|^{2} .
$$

Here $\xi$ and $\eta$ are the normal coordinates centered at $x_{\gamma, s}$ and $y_{\gamma s, s}$, and we consider $\sigma_{s}$ as a map from $T_{x_{\gamma, s}} X_{\gamma}$ to $T_{y_{\gamma s, s}} X_{\gamma s}$ as in Section 2. $\Gamma$ freely acts on $X^{\sharp(\Gamma, S)}$; for $g \in \Gamma$, we define $g: X_{\gamma}^{\prime} \rightarrow X_{g \gamma}^{\prime}$ by sending $p \in X_{\gamma}^{\prime}$ to $q \in X_{g \gamma}^{\prime}$ corresponding to the same point in $X$.

Let $g_{\gamma}(\gamma \in \Gamma)$ be the Riemannian metric on $X_{\gamma}$ which is the copy of the metric on $X$. Let $w$ be a smooth function in $X$ satisfying $0 \leq w \leq 1, w=1$ in the complement of the balls $B\left(x_{s}, \sqrt{\lambda}\right)$ and $B\left(y_{s}, \sqrt{\lambda}\right)(s \in S)$, and $w=0$ on each $B\left(x_{s}, \sqrt{\lambda} / 2\right)$ and $B\left(y_{s}, \sqrt{\lambda} / 2\right)$. We define a metric on $X^{\sharp(\Gamma, S)}$ by

$$
g:=\sum_{\gamma \in \Gamma} w_{\gamma} g_{\gamma}
$$

where the weight function $w_{\gamma}$ is the copy of $w$. We have

$$
X^{\sharp(\Gamma, S)}=\bigcup_{\gamma \in \Gamma} X_{\gamma}^{\prime}=\bigcup_{\gamma \in \Gamma} X_{\gamma}^{\prime \prime},
$$

and the Riemannian structure on $X^{\sharp(\Gamma, S)}$ is independent of $N$. Hence the above connected sum construction is compatible with that in Section 2.

The Riemannian metric $g$ is conformally equivalent to $g_{\gamma}$ over $X_{\gamma}^{\prime}\left(g=m_{\gamma}^{2} g_{\gamma}\right)$ and satisfies

$$
1 \leq m_{\gamma} \leq N^{2} \text { on } X_{\gamma}^{\prime}, \quad 1 \leq m_{\gamma} \leq 2^{3} \text { on } X_{\gamma}^{\prime \prime}
$$

Moreover, on each neighborhood of $x_{\gamma, s}$ and $y_{\gamma, s}$,

$$
N^{5 / 3} \leq m_{\gamma} \leq N^{2} \quad\left(\sqrt{\lambda} / N \leq|\xi| \leq \sqrt{\lambda} / N^{5 / 6}\right),
$$

where $\xi$ is the Euclidean coordinate (in $X_{\gamma}$ ) around $x_{\gamma, s}$ or $y_{\gamma, s}$.

The important point for the later argument is the following (essentially the same things are discussed in [5, pp. 293-294]): For a 1-form $\alpha$ and a 2-form $\xi$ on $X_{\gamma}^{\prime}$ we have

$$
|\alpha|_{g}^{q} d \operatorname{vol}_{g}=m_{r}^{4-q}|\alpha|_{g_{\gamma}}^{q} d \operatorname{vol}_{g_{\gamma}}, \quad|\xi|_{g}^{p} d \operatorname{vol}_{g}=m_{r}^{4-2 p}|\xi|_{g_{\gamma}}^{p} d \operatorname{vol}_{g_{\gamma}},
$$

where $d \mathrm{vol}_{g}$ denotes the volume form defined by $g$. Since $2<p<4$ and $q>4$, (7) implies

$$
\begin{aligned}
& \|\alpha\|_{L^{q}\left(X_{\gamma}^{\prime}, g\right)} \leq\|\alpha\|_{L^{q}\left(X_{\gamma}^{\prime}, g_{\gamma}\right)} \leq N^{2-8 / q}\|\alpha\|_{L^{p}\left(X_{\gamma}^{\prime}, g\right)}, \quad\|\alpha\|_{L^{q}\left(X_{\gamma}^{\prime \prime}, g_{\gamma}\right)} \leq 8^{1-4 / q}\|\alpha\|_{L^{q}\left(X_{\gamma}^{\prime \prime}, g\right)}, \\
& \|\xi\|_{L^{p}\left(X_{\gamma}^{\prime}, g\right)} \leq\|\xi\|_{L^{p}\left(X_{\gamma}^{\prime}, g_{\gamma}\right)} \leq N^{4-8 / p}\|\xi\|_{L^{p}\left(X_{\gamma}^{\prime}, g\right)}, \quad\|\xi\|_{L^{p}\left(X_{\gamma}^{\prime \prime}, g_{\gamma}\right)} \leq 8^{2-4 / p}\|\xi\|_{L^{p}\left(X_{\gamma}^{\prime \prime}, g\right)} .
\end{aligned}
$$


3.2. Gluing principal $S U(2)$-bundles. Let $M$ be a set of (not necessarily all) gauge equivalence classes of $(E, A)$, where $E$ is a principal $S U(2)$-bundle over $X$ and $A$ is an ASD connection on $E$. We suppose that $M$ can be decomposed as $M=M_{0} \sqcup M_{1}$ (disjoint union) satisfying the following conditions:

(a) $M_{0}$ and $M_{1}$ are compact with respect to the topology of $\mathcal{C}^{\infty}$-convergence. (In particular, the number of the possible topological types of $E$ is finite.)

(b) For all $[E, A] \in M_{0}, A$ is a regular connection. That is, the following two conditions are satisfied: $A$ is irreducible (i.e., if a gauge transformation $g: E \rightarrow E$ satisfies $g(A)=A$, then $g= \pm 1)$ and the operator $d_{A}^{+}: \Omega^{1}(\operatorname{ad} E) \rightarrow \Omega^{+}(\operatorname{ad} E)$ is surjective. Here $\Omega^{1}$ is the space of 1 -forms and $\Omega^{+}$is the space of self-dual forms. ad $E$ is the Lie algebra bundle associated with $E$.

(c) If $X$ satisfies $b_{1}(X)=b^{+}(X)=0$ (e.g., $X=S^{4}, \overline{\mathbb{C} P^{2}}$ ), then

$$
M_{1} \subset\{[X \times S U(2) \text {, the product connection }]\} .
$$

Otherwise we set $M_{1}=\emptyset$. Therefore $M_{1}$ is the one-point space or empty.

Remark 3.1. Let $E:=X \times S U(2)$ and $A$ be the product connection. If $b^{+}(X)=0$, then $d_{A}^{+}: \Omega^{1}(\operatorname{ad} E) \rightarrow \Omega^{+}(\operatorname{ad} E)$ is surjective. But $A$ is not irreducible. All constant gauge transformations fix $A$. The condition $b^{+}(X)=b_{1}(X)=0$ implies that $[A]$ has no local deformation as an ASD connection.

In our application to Theorem 2.3, we need to consider the product connection. The condition (c) is added for this purpose. But if the reader does not want to consider reducible connections, you should consider only the case $M=M_{0}$.

Definition 3.2. A sequence $\left(E_{\gamma}, A_{\gamma}, \rho_{\gamma, s}\right)_{\gamma \in \Gamma, s \in S}$ is called a gluing data (or $M$-gluing data) if it satisfies the following:

(i) For all $\gamma \in \Gamma, E_{\gamma}$ is a principal $S U(2)$-bundle over $X_{\gamma}$ and $A_{\gamma}$ is an ASD connection on it which satisfies $\left[E_{\gamma}, A_{\gamma}\right] \in M$. (Here we naturally identify $X_{\gamma}$ with $X$.)

(ii) $\rho_{\gamma, s}:\left(E_{\gamma}\right)_{x_{\gamma, s}} \rightarrow\left(E_{\gamma s}\right)_{y_{\gamma s, s}}(\gamma \in \Gamma, s \in S)$ is an $S U(2)$-isomorphism between the fibers $\left(E_{\gamma}\right)_{x_{\gamma, s}}$ and $\left(E_{\gamma s}\right)_{y_{\gamma, s}}$. We call $\rho=\left(\rho_{\gamma, s}\right)_{\gamma \in \Gamma, s \in S}$ "gluing parameter".

We consider that two gluing data $\left(E_{1 \gamma}, A_{1 \gamma}, \rho_{1 \gamma, s}\right)_{\gamma \in \Gamma, s \in S}$ and $\left(E_{2 \gamma}, A_{2 \gamma}, \rho_{2 \gamma, s}\right)_{\gamma \in \Gamma, s \in S}$ are equivalent if there exist bundle isomorphisms $g_{\gamma}: E_{1 \gamma} \rightarrow E_{2 \gamma}(\gamma \in \Gamma)$ satisfying $g_{\gamma}\left(A_{1 \gamma}\right)=$ $A_{2 \gamma}$ and $g_{\gamma s} \rho_{1 \gamma, s}=\rho_{2 \gamma, s} g_{\gamma}$ (in particular, $E_{1 \gamma}$ and $E_{2 \gamma}$ are isomorphic). We define GID as the set of the equivalence classes of gluing data. (We sometimes use the notation " $\mathrm{GlD}_{M}$ " when we need to make the dependence on $M$ explicit.) There exists a natural projection GlD $\rightarrow M^{\Gamma}$ defined by $\left(E_{\gamma}, A_{\gamma}, \rho_{\gamma, s}\right)_{\gamma \in \Gamma, s \in S} \mapsto\left(E_{\gamma}, A_{\gamma}\right)_{\gamma \in \Gamma}$.

Let $\theta=\left(E_{\gamma}, A_{\gamma}, \rho_{\gamma, s}\right)_{\gamma \in \Gamma, s \in S}$ be a gluing data. For $x \in X_{\gamma}$ in a small neighborhood of $x_{\gamma, s}$, the fiber of $E_{\gamma}$ over the point $x$ can be identified with the fiber over $x_{\gamma, s}$ by using the parallel transport (defined by $A_{\gamma}$ ) along the radial line from $x_{\gamma, s}$ to $x$. This trivialization is usually called "exponential gauge" (or sometimes "radial gauge"); see [6, Chapter 9] or 
[5, Section 2.3.1]. Using these exponential gauges centered at $x_{\gamma, s}$ or $y_{\gamma, s}$, we trivialize the bundle $E_{\gamma}$ over $\bigsqcup_{s \in S} \Omega\left(x_{\gamma, s}\right) \sqcup \Omega\left(y_{\gamma, s}\right)$. $\left(\Omega\left(x_{\gamma, s}\right)\right.$ and $\Omega\left(y_{\gamma, s}\right)$ are the annulus regions over $X_{\gamma}$ defined in Section 3.1: $\Omega\left(x_{\gamma, s}\right)=B\left(x_{\gamma, s}, N \sqrt{\lambda}\right) \backslash \bar{B}\left(x_{\gamma, s}, \sqrt{\lambda} / N\right)$.)

Each $\rho_{\gamma, s}$ is an isomorphism between $\left(E_{\gamma}\right)_{x_{\gamma, s}}$ and $\left(E_{\gamma_{s}}\right)_{y_{\gamma s, s}}$. We have the identification $\Omega\left(x_{\gamma, s}\right) \cong \Omega\left(y_{\gamma s, s}\right)$ defined by (6), and the above exponential gauges give the bundle trivializations $\left.E_{\gamma}\right|_{\Omega\left(x_{\gamma, s}\right)} \cong \Omega\left(x_{\gamma, s}\right) \times\left(E_{\gamma}\right)_{x_{\gamma, s}}$ and $\left.E_{\gamma s}\right|_{\Omega\left(y_{\gamma s, s}\right)} \cong \Omega\left(y_{\gamma s, s}\right) \times\left(E_{\gamma s}\right)_{y_{\gamma s, s}}$. Therefore $\rho_{\gamma, s}$ gives an identification map between $\left.E_{\gamma}\right|_{\Omega\left(x_{\gamma, s}\right)}$ and $\left.E_{\gamma s}\right|_{\Omega\left(y_{\gamma s, s}\right)}$ covering the base space identification $\Omega\left(x_{\gamma, s}\right) \cong \Omega\left(y_{\gamma s, s}\right)$ (see the diagram (11)).

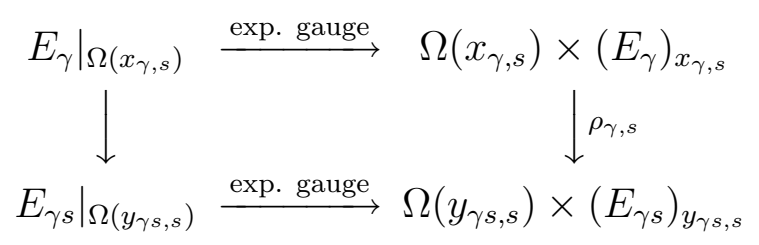

We define a principal $S U(2)$ bundle $\boldsymbol{E}(\theta)$ over $X^{\sharp(\Gamma, S)}$ by setting

$$
\boldsymbol{E}(\theta):=\left(\left.\bigsqcup_{\gamma \in \Gamma} E_{\gamma}\right|_{X_{\gamma}^{\prime}}\right) / \sim,
$$

where we identify $\left.E_{\gamma}\right|_{\Omega\left(x_{\gamma, s}\right)}$ with $\left.E_{\gamma s}\right|_{\Omega\left(y_{\gamma s, s}\right)}$ by (11).

3.3. Cut-off functions. We need to introduce several cut-off functions. We basically follow the description of Donaldson-Kronheimer [5, Section 7.2]. First note that the following fact. Since $M$ is compact, there exists an uniform upper bound of $\left|F_{A}\right|$ for all $[E, A] \in M$ :

$$
\left|F_{A}\right| \leq \text { const }_{M}
$$

where const $_{M}$ denotes a positive constant depending only on $M$.

Set $b:=4 N \sqrt{\lambda}(\ll 1)$. Let $\psi$ be the cut-off function on $X$ such that $\psi=0$ over $\bigsqcup_{s \in S} B\left(x_{s}, b / 2\right) \sqcup B\left(y_{s}, b / 2\right)$ and $\psi=1$ over the complement of $\bigsqcup_{s \in S} B\left(x_{s}, b\right) \sqcup B\left(y_{s}, b\right)$ and $|d \psi| \leq 4 / b$. Let $\psi_{\gamma}$ be the copy of $\psi$ defined on $X_{\gamma}$.

Let $\theta=\left(E_{\gamma}, A_{\gamma}, \rho_{\gamma, s}\right)_{\gamma \in \Gamma, s \in S}$ be a gluing data. As in Section 3.2, we trivialize the bundle $E_{\gamma}$ around $x_{\gamma, s}$ and $y_{\gamma, s}$ by using the exponential gauges. Then we can define a connection $A_{\gamma}^{\prime}$ on $E_{\gamma}$ by setting

$$
A_{\gamma}^{\prime}:=\psi_{\gamma} A_{\gamma}
$$

Here we consider $A_{\gamma}$ as a connection matrix over each neighborhood of $x_{\gamma, s}$ and $y_{\gamma, s}$ by using the above trivialization. In the exponential gauge we have $\left|A_{\gamma}(x)\right| \leq|x| \sup \left|F_{A_{\gamma}}\right| \leq$ const $_{M}|x|$ (see Donaldson-Kronheimer [5, p. 54]). Therefore we have

$$
\left|A_{\gamma}^{\prime}-A_{\gamma}\right| \leq \text { const }_{M} \cdot b, \quad\left|F^{+}\left(A_{\gamma}^{\prime}\right)\right| \leq \text { const }_{M}, \quad\left|F\left(A_{\gamma}^{\prime}\right)-F\left(A_{\gamma}\right)\right| \leq \text { const }_{M},
$$


where const $M$ is a positive constant which only depends on $M$ (and is independent of $\gamma$, $b, \lambda, N)$. Then

$$
\begin{aligned}
& \left\|A_{\gamma}^{\prime}-A_{\gamma}\right\|_{L^{4}\left(X_{\gamma}, g_{\gamma}\right)} \leq \operatorname{const}_{M} \cdot b^{2}, \quad\left\|F^{+}\left(A_{\gamma}^{\prime}\right)\right\|_{L^{p}\left(X_{\gamma}, g_{\gamma}\right)} \leq \operatorname{const}_{M} \cdot b^{4 / p}, \\
& \left\|F\left(A_{\gamma}^{\prime}\right)-F\left(A_{\gamma}\right)\right\|_{L^{p}\left(X_{\gamma}, g_{\gamma}\right)} \leq \operatorname{const}_{M} \cdot b^{4 / p} .
\end{aligned}
$$

$A_{\gamma}^{\prime}$ and $A_{\gamma s}^{\prime}(s \in S)$ coincide with each other over $X_{\gamma}^{\prime} \cap X_{\gamma s}^{\prime}$ under the identification (11). Hence there exists an unique (not necessarily ASD) connection $\boldsymbol{A}^{\prime}(\theta)$ on $\boldsymbol{E}(\theta)$ compatible with each $A_{\gamma}^{\prime}$ over $X_{\gamma}^{\prime}$.

Remark 3.3. If $\left[E_{\gamma}, A_{\gamma}\right] \in M_{1}$ (i.e., $A_{\gamma}$ is gauge equivalent to the product connection), then $A_{\gamma}^{\prime}=A_{\gamma}$. Hence if $\left[E_{\gamma}, A_{\gamma}\right] \in M_{1}$ for all $\gamma \in \Gamma$, then $\boldsymbol{A}^{\prime}(\theta)$ is a flat connection on $\boldsymbol{E}(\theta)$ (which might have a non-trivial holonomy).

Later (in Section 5.1) we will need the following $\left\{\psi_{\gamma}^{\prime}\right\}$ also; Let $\psi^{\prime}$ be the cut-off function on $X$ such that $\psi^{\prime}=0$ over $\bigsqcup_{s \in S} B\left(x_{s}, b / 4\right) \sqcup B\left(y_{s}, b / 4\right)$ and $\psi^{\prime}=1$ over the complement of $\bigsqcup_{s \in S} B\left(x_{s}, b / 2\right) \sqcup B\left(y_{s}, b / 2\right)$ and $\left|d \psi^{\prime}\right| \leq 8 / b$. Let $\psi_{\gamma}^{\prime}$ be the copy of $\psi^{\prime}$ defined on $X_{\gamma}$.

The following lemma is essentially the copy of [5, Lemma (7.2.10)]:

Lemma 3.4. There exists a positive number $K$ satisfying the following: For any $\lambda$ and $N$ there exists a smooth function $\beta=\beta_{\lambda, N}$ defined in $\mathbb{R}^{4}$ such that $\beta(x)=0$ for $|x| \leq \sqrt{\lambda} / N$, $\beta(x)=1$ for $|x| \geq \sqrt{\lambda} / N^{5 / 6}$ and

$$
\|d \beta\|_{L^{4}} \leq K(\log N)^{-3 / 4}
$$

Proof. Note that the $L^{4}$-norm of a 1 -form is conformally invariant. So we can change the description from the Euclidean $\mathbb{R}^{4}$ to the cylinder $S^{3} \times \mathbb{R}$ by the coordinate transform $t=\log |x|-\log \sqrt{\lambda} \cdot \sqrt{\lambda} / N \leq|x| \leq \sqrt{\lambda} / N^{5 / 6}$ becomes $-\log N \leq t \leq-(5 / 6) \log N$. Then the proof is easy.

The condition $\operatorname{supp}(d \beta) \subset\left\{\sqrt{\lambda} / N \leq|x| \leq \sqrt{\lambda} / N^{5 / 6}\right\}$ will be used in Section 6.1 (cf. (8)). Using the above Lemma 3.4, we define a cut-off function $\beta$ on $X$ by putting the above $\beta_{\lambda, N}$ around each $x_{\gamma}$ and $y_{\gamma}$. That is, $\beta$ is a function with $0 \leq \beta \leq 1$ such that $\beta=0$ on $\bigsqcup_{s \in S} B\left(x_{s}, \sqrt{\lambda} / N\right) \sqcup B\left(y_{s}, \sqrt{\lambda} / N\right), \beta=1$ on the complement of $\bigsqcup_{s \in S} B\left(x_{s}, \sqrt{\lambda} / N^{5 / 6}\right) \sqcup$ $B\left(y_{s}, \sqrt{\lambda} / N^{5 / 6}\right)$ and

$$
\|d \beta\|_{L^{4}} \leq K(\log N)^{-3 / 4}
$$

(Strictly speaking, the above constant $K$ should be $(2|S|)^{1 / 4} K$. But for simplicity we use the abuse of notation.) Let $\beta_{\gamma}$ be the copy of $\beta$ defined on $X_{\gamma}$.

We need to introduce one more cut-off. Let $\beta^{\prime}$ be a smooth function on $X$ such that $0 \leq \beta^{\prime} \leq 1, \beta^{\prime}=0$ on $\bigsqcup_{s \in S} B\left(x_{s}, \sqrt{\lambda} / 2\right) \sqcup B\left(y_{s}, \sqrt{\lambda} / 2\right), \beta^{\prime}=1$ on the complement of $\bigsqcup_{s \in S} B\left(x_{s}, 2 \sqrt{\lambda}\right) \sqcup B\left(y_{s}, 2 \sqrt{\lambda}\right)$ (hence $\left.\operatorname{supp} \beta^{\prime} \subset X^{\prime \prime}\right)$. We can choose $\beta^{\prime}$ so that the $L^{4}$ norm $\left\|d \beta^{\prime}\right\|_{L^{4}}$ is independent of $\lambda\left(\right.$ and $N$ ). Since $N \gg 1$, we have $\sqrt{\lambda} / 2 \geq \sqrt{\lambda} / N^{5 / 6}$ and 
hence

$$
\beta \cdot \beta^{\prime}=\beta^{\prime}
$$

Let $\beta_{\gamma}^{\prime}$ be the copy of $\beta$ defined on $X_{\gamma}\left(\beta_{\gamma} \cdot \beta_{\gamma}^{\prime}=\beta_{\gamma}^{\prime}\right)$. Moreover we choose $\beta^{\prime}$ so that these

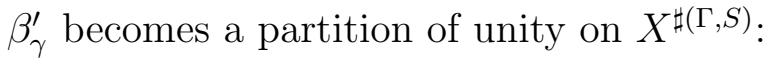

$$
\sum_{\gamma \in \Gamma} \beta_{\gamma}^{\prime}=1
$$

In particular we have $\beta_{\gamma}^{\prime}+\beta_{\gamma s}^{\prime}=1$ over $\Omega\left(x_{\gamma, s}\right)=\Omega\left(y_{\gamma s, s}\right)$.

3.4. Preliminary estimates. In this subsection we prepare several estimates. I think that they are essentially well-known. Therefore we omit most of the proofs. If some readers feel this subsection cumbersome, you should skip it and return to this subsection when it is used.

3.4.1. Right inverse of $d_{A}^{+}$. Let $[E, A] \in M$, and set $\Delta_{A}:=\left(d_{A}^{+}\right)\left(d_{A}^{+}\right)^{*}: \Omega^{+}(\operatorname{ad} E) \rightarrow$ $\Omega^{+}(\operatorname{ad} E)$, where $\left(d_{A}^{+}\right)^{*}: \Omega^{+}(\operatorname{ad} E) \rightarrow \Omega^{1}(\operatorname{ad} E)$ is the formal adjoint of $d_{A}^{+}$. From the conditions (b) and (c) in the beginning of Section 3.2, there exists the inverse $\Delta_{A}^{-1}$ (see also Remark 3.1). Set $P_{A}:=\left(d_{A}^{+}\right)^{*} \cdot \Delta_{A}^{-1}: \Omega^{+}(\operatorname{ad} E) \rightarrow \Omega^{1}(\operatorname{ad} E) . \quad P_{A}$ becomes a right inverse of $d_{A}^{+}: d_{A}^{+} P_{A}=1$.

Remember that $2<p<4, q>4$ and $1-4 / p=-4 / q$. We have the Sobolev embedding: $L_{1}^{p}(X) \hookrightarrow L^{q}(X)$. Since $M$ is compact, there exists a positive constant const ${ }_{M}$ depending only on $M$ (and independent of $A$ ) such that

$$
\left\|P_{A} \xi\right\|_{L^{q}} \leq \text { const }_{M}\|\xi\|_{L^{p}}, \quad\left\|d_{A} P_{A}(\xi)\right\|_{L^{p}} \leq \operatorname{const}_{M}\|\xi\|_{L^{p}} .
$$

for any $[E, A] \in M$ and any $\xi \in \Omega^{+}(\operatorname{ad} E)$.

3.4.2. The cohomology $H_{A}^{1}$. Let $[E, A] \in M$ and set

$$
H_{A}^{1}:=\operatorname{ker}\left(d_{A}^{*}+d_{A}^{+}: \Omega^{1}(\operatorname{ad} E) \rightarrow\left(\Omega^{0} \oplus \Omega^{+}\right)(\operatorname{ad} E)\right) .
$$

(If $[E, A] \in M_{1}$, then $H_{A}^{1}=0$.) There exists $\delta_{M}>0$ such that for any $\alpha \in H_{A}^{1}$ with $\|\alpha\|_{L^{q}} \leq \delta_{M}$ we have $\tilde{\alpha}=\tilde{\alpha}(A, \alpha) \in \Omega^{1}(\operatorname{ad} E)$ satisfying the following:

$$
\begin{gathered}
d_{A}^{*} \tilde{\alpha}=0, \quad F^{+}(A+\tilde{\alpha})=0, \\
\|\tilde{\alpha}-\alpha\|_{L^{q}} \leq \operatorname{const}_{M}\|\alpha\|_{L^{q}}^{2} .
\end{gathered}
$$

(We have $\tilde{\alpha}(A, 0)=0$.) Moreover

$$
\|\alpha\|_{L^{q}} \leq \operatorname{const}_{M} \cdot d_{L^{q}}([A],[A+\tilde{\alpha}]) .
$$

Here, for connections $A_{1}$ and $A_{2}$ on $E$, we define $L^{q}$-distance $d_{L^{q}}\left(\left[A_{1}\right],\left[A_{2}\right]\right)$ by

$$
d_{L^{q}}\left(\left[A_{1}\right],\left[A_{2}\right]\right):=\inf _{g: E \rightarrow E}\left\|A_{2}-g\left(A_{1}\right)\right\|_{L^{q}(X)} .
$$


Lemma 3.5. There is $\delta_{M}^{\prime}>0$ such that if an $A S D$ connection $B$ on $E$ with $[E, B] \in M$ satisfies $d_{L^{q}}([A],[B]) \leq \delta_{M}^{\prime}$ then there exists $\alpha \in H_{A}^{1}$ with $\|\alpha\|_{L^{q}} \leq \delta_{M}$ satisfying $[B]=$ $[A+\tilde{\alpha}]$.

Lemma 3.6. If we choose $\delta_{M}$ sufficiently small, then for any $\xi \in \Omega^{+}(\operatorname{ad} E)$ and $\alpha \in H_{A}^{1}$ with $\|\alpha\|_{L^{q}} \leq \delta_{M}$,

$$
\left\|P_{A}(\xi)-P_{A+\tilde{\alpha}}(\xi)\right\|_{L^{q}} \leq \text { const }_{M}\|\alpha\|_{L^{q}}\|\xi\|_{L^{p}} .
$$

3.4.3. Auxiliary estimates. For $\varepsilon>0$ let $X_{\varepsilon} \subset X$ be the complement of the union of the balls $\bar{B}\left(x_{s}, \varepsilon\right)$ and $\bar{B}\left(y_{s}, \varepsilon\right)(s \in S)$ :

$$
X_{\varepsilon}:=X \backslash\left(\bigcup_{s \in S} \bar{B}\left(x_{s}, \varepsilon\right) \cup \bar{B}\left(y_{s}, \varepsilon\right)\right) .
$$

Lemma 3.7. There is $\varepsilon_{M}>0$ such that if $\varepsilon \leq \varepsilon_{M}$ then for any two $\left[E_{i}, A_{i}\right] \in M(i=1,2)$ we have the following:

(1) If $E_{1}$ is isomorphic to $E_{2}$, then

$$
d_{L^{q}}\left(\left[A_{1}\right],\left[A_{2}\right]\right) \leq \operatorname{const}_{M} \cdot d_{L^{q}}\left(\left[\left.A_{1}\right|_{X_{\varepsilon}}\right],\left[\left.A_{2}\right|_{X_{\varepsilon}}\right]\right),
$$

where $d_{L^{q}}\left(\left[A_{1} \mid X_{\varepsilon}\right],\left[A_{2} \mid X_{\varepsilon}\right]\right)$ is given by

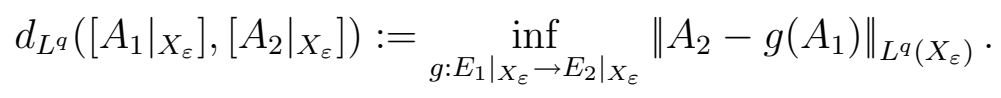

(2) If $E_{1}$ is not isomorphic to $E_{2}$, then

$$
d_{L^{q}}\left(\left[\left.A_{1}\right|_{X_{\varepsilon}}\right],\left[\left.A_{2}\right|_{X_{\varepsilon}}\right]\right) \geq \text { const }_{M}>0 .
$$

For $[E, A] \in M$ we denote $I_{A}$ as the set of gauge transformations $g: E \rightarrow E$ satisfying $g(A)=A$. If $[E, A] \in M_{0}$, then $I_{A}=\{ \pm 1\}$, and if $[E, A] \in M_{1}$, then $I_{A} \cong S U(2)$ (the set of constant gauge transformations).

Lemma 3.8. There is $\varepsilon_{M}^{\prime}>0$ such that if $\varepsilon \leq \varepsilon_{M}^{\prime}$ then we have the following: Let $[E, A] \in M$ and $g:\left.\left.E\right|_{X_{\varepsilon}} \rightarrow E\right|_{X_{\varepsilon}}$ be a bundle map over $X_{\varepsilon}$. Then

$$
\min _{h \in I_{A}}\|g-h\|_{\mathcal{C}^{0}\left(X_{\varepsilon}\right)} \leq \text { const }_{M}\left\|d_{A} g\right\|_{L^{q}\left(X_{\varepsilon}\right)} .
$$

3.4.4. Estimates about the exponential gauge. Let $D \subset \mathbb{R}^{4}$ be a ball centered at the origin in the Euclidean space $\mathbb{R}^{4}$, and $E=D \times S U(2)$ be a principal $S U(2)$ bundle over $D$ with smooth (not necessarily ASD) connections $A_{1}$ and $A_{2}$. Let $u_{i}: E \rightarrow D \times E_{0}$ $(i=1,2)$ be the exponential gauges associated with $A_{i}$ centered at the origin. $\left(E_{0}\right.$ is the fiber of $E$ at the origin.) We have $\partial u_{i} / \partial r=u_{i} A_{i, r}(r=|x|)$ and hence $\partial\left(u_{1} u_{2}^{-1}\right) / \partial r=$ $u_{1}\left(A_{1, r}-A_{2, r}\right) u_{2}^{-1}$. Therefore

$$
\left|u_{1}(x)-u_{2}(x)\right| \leq|x| \cdot\left\|A_{1}-A_{2}\right\|_{\mathcal{C}^{0}(B)} .
$$

Let $B_{i}:=u_{i}\left(A_{i}\right)$ be the connection matrices in the exponential gauge $(i=1,2)$. 


\section{Lemma 3.9.}

$$
\left|B_{1}-B_{2}\right| \leq r\left\|F\left(A_{1}\right)-F\left(A_{2}\right)\right\|_{\mathcal{C}^{0}}+\frac{r^{2}}{2}\left\|A_{1}-A_{2}\right\|_{\mathcal{C}^{0}}\left(\left\|F\left(A_{1}\right)\right\|_{\mathcal{C}^{0}}+\left\|F\left(A_{2}\right)\right\|_{\mathcal{C}^{0}}\right) .
$$

Proof. We have

$$
B_{i, \theta}=\int_{0}^{r} F\left(B_{i}\right)_{r \theta} d r=\int_{0}^{r} u_{i} F\left(A_{i}\right)_{r \theta} u_{i}^{-1} d r
$$

where $r, \theta$ denote the polar coordinate. (Of course, $\theta$ has three components.) Hence

$$
\begin{aligned}
& B_{1, \theta}-B_{2, \theta}= \\
& \int_{0}^{r}\left\{\left(u_{1}-u_{2}\right) F\left(A_{1}\right)_{r \theta} u_{1}^{-1}+u_{2}\left(F\left(A_{1}\right)_{r \theta}-F\left(A_{2}\right)_{r \theta}\right) u_{1}^{-1}+u_{2} F\left(A_{2}\right)_{r \theta}\left(u_{1}^{-1}-u_{2}^{-1}\right)\right\} d r .
\end{aligned}
$$

Then

$$
\begin{aligned}
\left|B_{1}-B_{2}\right| & \leq \int_{0}^{r}\left|u_{1}-u_{2}\right|\left(\left|F\left(A_{1}\right)\right|+\left|F\left(A_{2}\right)\right|\right)+\left|F\left(A_{1}\right)-F\left(A_{2}\right)\right| d r \\
& \leq \frac{r^{2}}{2}\left\|A_{1}-A_{2}\right\|_{\mathcal{C}^{0}}\left(\left\|F\left(A_{1}\right)\right\|_{\mathcal{C}^{0}}+\left\|F\left(A_{2}\right)\right\|_{\mathcal{C}^{0}}\right)+r\left\|F\left(A_{1}\right)-F\left(A_{2}\right)\right\|_{\mathcal{C}^{0}} .
\end{aligned}
$$

Let $A=A(t)$ be a family of connections on $E$ depending smoothly on the parameter $t \in(-1,1)$. Let $u=u(t): E \rightarrow D \times E_{0}$ be the exponential gauge of $A$ about the origin, and set $B=B(t)=u(A)$. Suppose that there exists a family of sections $w=w(t)$ of $\operatorname{ad} E$ such that $u(t)=u(0) e^{w(t)}$ and $w(0) \equiv 0$. Let $\psi$ and $\phi$ be smooth functions on $D$ satisfying $0 \leq \phi, \psi \leq 1$. Set $A_{1}=A_{1}(t):=u^{-1}(\psi B)$ (we consider $B$ as a connection matrix), and $A_{2}=A_{2}(t):=e^{\phi w}\left(A_{1}(t)\right)$.

\section{Lemma 3.10.}

$$
\left|\frac{\partial A_{2}}{\partial t}\right|_{t=0}\left|\leq\left(1+r|d \phi|+3 r^{2}\|F(A(0))\|_{\mathcal{C}^{0}}\right)\left\|\left.\frac{\partial A}{\partial t}\right|_{t=0}\right\|_{\mathcal{C}^{0}}+r\left\|\left.d_{A}\left(\frac{\partial A}{\partial t}\right)\right|_{t=0}\right\|_{\mathcal{C}^{0}} .\right.
$$

Proof. We can assume that $A(0)$ is already a connection matrix in the exponential gauge. Then $u(0) \equiv 1, u(t)=e^{w(t)}$ and $B(0) \equiv A(0)$. Let $(r, \theta)$ be the polar coordinate. Set $A_{r}=A_{r}(t):=\langle A(t), \partial / \partial r\rangle$. We have $A_{r}(0) \equiv 0$ and $\partial u / \partial r=u A_{r}$. Hence

$$
\left.\frac{\partial}{\partial r}\left(\frac{\partial u}{\partial t}\right)\right|_{t=0}=\left.u \frac{\partial A_{r}}{\partial t}\right|_{t=0} .
$$

Since $u=1$ at the origin for all $t$, we have $\partial u / \partial t=0$ at the origin. Hence

$$
\left|\frac{\partial u}{\partial t}\right|_{t=0}\left|\leq r\left\|\left.\frac{\partial A}{\partial t}\right|_{t=0}\right\|_{\mathcal{C}^{0}} .\right.
$$

We have

$$
B_{\theta}=\int_{0}^{r} F(B)_{r \theta} d r=\int_{0}^{r} u F(A)_{r \theta} u^{-1} d r
$$


Differentiating this equation and using the above (22), we get

$$
\left|\frac{\partial B}{\partial t}\right|_{t=0}\left|\leq r^{2}\|F(A(0))\|_{\mathcal{C}^{0}}\left\|\left.\frac{\partial A}{\partial t}\right|_{t=0}\right\|_{\mathcal{C}^{0}}+r\left\|\left.d_{A}\left(\frac{\partial A}{\partial t}\right)\right|_{t=0}\right\|_{\mathcal{C}^{0}} .\right.
$$

We have $d_{A} u=(A-B) u$. Differentiating this (and using $u(0) \equiv 1$ ), we get

$$
\left.d_{A}\left(\frac{\partial u}{\partial t}\right)\right|_{t=0}=\left.\left(\frac{\partial A}{\partial t}-\frac{\partial B}{\partial t}\right)\right|_{t=0} .
$$

We have $A_{1}=u^{-1}(\psi B)=u^{-1} d u+\psi u^{-1} B u$ and $A(0)=B(0)$. Then

$$
\begin{aligned}
\left.\frac{\partial A_{1}}{\partial t}\right|_{t=0} & =\left.d_{A}\left(\frac{\partial u}{\partial t}\right)\right|_{t=0}+\left.(\psi-1)[A, \partial u / \partial t]\right|_{t=0}+\left.\psi \frac{\partial B}{\partial t}\right|_{t=0}, \\
& =\left.\frac{\partial A}{\partial t}\right|_{t=0}+\left.(\psi-1) \frac{\partial B}{\partial t}\right|_{t=0}+\left.(\psi-1)[A, \partial u / \partial t]\right|_{t=0} .
\end{aligned}
$$

We have $w(0) \equiv 0, \partial w /\left.\partial t\right|_{t=0}=\partial u /\left.\partial t\right|_{t=0}$ and $A_{1}(0)=\psi B(0)=\psi A(0)$. Some calculation shows

$$
\begin{aligned}
\left.\frac{\partial A_{2}}{\partial t}\right|_{t=0}= & -\left.d \phi \otimes \frac{\partial u}{\partial t}\right|_{t=0}+\left.(1-\phi) \frac{\partial A}{\partial t}\right|_{t=0} \\
& +\left.(\psi+\phi-1) \frac{\partial B}{\partial t}\right|_{t=0}+\left.(\psi-1)(1-\phi)[A, \partial u / \partial t]\right|_{t=0} .
\end{aligned}
$$

We have $|A(0)| \leq r\|F(A(0))\|_{\mathcal{C}^{0}}$. Therefore

$$
\left|\frac{\partial A_{2}}{\partial t}\right|_{t=0}\left|\leq\left(1+r|d \phi|+3 r^{2}\|F(A(0))\|_{\mathcal{C}^{0}}\right)\left\|\left.\frac{\partial A}{\partial t}\right|_{t=0}\right\|_{\mathcal{C}^{0}}+r\left\|\left.d_{A}\left(\frac{\partial A}{\partial t}\right)\right|_{t=0}\right\|_{\mathcal{C}^{0}} .\right.
$$

Moreover suppose that $A=A(t)$ is a family of ASD connections. We have $F^{+}\left(A_{1}\right)=$ $u^{-1} F^{+}(\psi B) u$ and $F^{+}(\psi B)=(d \psi \wedge B)^{+}+\psi(\psi-1)(B \wedge B)^{+}$. Using the inequalities $(22)$ and (23), we get (at $t=0)$

$$
\begin{aligned}
\left|\frac{\partial}{\partial t} F^{+}\left(A_{1}\right)\right| & \leq 2 r\left|F^{+}(\psi B)\right|\left\|\frac{\partial A}{\partial t}\right\|_{\mathcal{C}^{0}}+\left|\frac{\partial}{\partial t} F^{+}(\psi B)\right| \\
\left|F^{+}(\psi B)\right| & \leq\left(r|d \psi|+r^{2}\left\|F_{A}\right\|_{\mathcal{C}^{0}}\right)\left\|F_{A}\right\|_{\mathcal{C}^{0}} \\
\left|\frac{\partial}{\partial t} F^{+}(\psi B)\right| & \leq\left(r|d \psi|+2 \sqrt{2} r^{2}\left\|F_{A}\right\|_{\mathcal{C}^{0}}\right)\left(r\left\|F_{A}\right\|_{\mathcal{C}^{0}}\left\|\frac{\partial A}{\partial t}\right\|_{\mathcal{C}^{0}}+\left\|d_{A}\left(\frac{\partial A}{\partial t}\right)\right\|_{\mathcal{C}^{0}}\right) .
\end{aligned}
$$

\section{INFINITE GLUING: BASIC CONSTRUCTION}

In the following three sections we will develop the technique of gluing an infinite number of ASD connections. Our approach is based on the method of Donaldson-Kronheimer [5, Section 7.2]. We also use the ideas of Angenent [1] and Macrì-Nolasco-Ricciardi [11] in the construction of the right inverse of $d_{\boldsymbol{A}^{\prime}}^{+}$. A different approach using "alternating method" in Donaldson [4] is developed in Tsukamoto [13]. Recall that $2<p<4, q>4$ and $1-4 / p=-4 / q$. 
4.1. Construction. Let $\theta=\left(E_{\gamma}, A_{\gamma}, \rho_{\gamma, s}\right)_{\gamma \in \Gamma, s \in S}$ be a gluing data. That is, $E_{\gamma}(\gamma \in \Gamma)$ is a principal $S U(2)$ bundle over $X_{\gamma}$, and $A_{\gamma}$ is an ASD connection on $E_{\gamma}$ satisfying $\left[E_{\gamma}, A_{\gamma}\right] \in M . \rho:=\left(\rho_{\gamma, s}\right)_{\gamma \in \Gamma, s \in S}$ is a gluing parameter. We have constructed the principal $S U(2)$ bundle $\boldsymbol{E}=\boldsymbol{E}(\theta)$ on $X^{\sharp(\Gamma, S)}$. We want to construct an ASD connection on $\boldsymbol{E}$ by gluing the given ASD connections $A_{\gamma}$.

Let $\alpha$ and $\xi$ be ad $\boldsymbol{E}$-valued 1-form and self-dual 2-form on $X^{\sharp(\Gamma, S)}$ respectively. We define $B L^{q}$-norm (bounded $L^{q}$-norm) of $\alpha$ and $B L^{p}$-norm of $\xi$ by

$$
\|\alpha\|_{B L^{q}}:=\sup _{\gamma \in \Gamma}\|\alpha\|_{L^{q}\left(X_{\gamma}^{\prime \prime}, g\right)}, \quad\|\xi\|_{B L^{p}}:=\sup _{\gamma \in \Gamma}\|\xi\|_{L^{p}\left(X_{\gamma}^{\prime \prime}, g\right)} \cdot
$$

Let $B L^{q}$ be the Banach space of all locally- $L^{q}$, ad $\boldsymbol{E}$-valued 1-forms whose $B L^{q}$-norms are finite, and $B L^{p}$ be the Banach space of all locally- $L^{p}, \operatorname{ad} \boldsymbol{E}$-valued self-dual 2-forms whose $B L^{p}$-norms are finite. This type of function space is used in Macrí-Nolasco-Ricciardi [11] for the study of self-dual vortices. It is also used in Gournay [7] for the study of gluing infinitely many pseudo-holomorphic curves. For $\xi \in B L^{p}$ we define an ad $\boldsymbol{E}$-valued 1-form $Q(\xi)=Q_{\theta}(\xi)$ by

$$
Q(\xi):=\sum_{\gamma \in \Gamma} \beta_{\gamma} P_{A_{\gamma}}\left(\beta_{\gamma}^{\prime} \xi\right)
$$

where $P_{A_{\gamma}}$ is the right inverse of $d_{A_{\gamma}}^{+}$defined in Section 3.4.1. The above infinite sum is a locally finite sum, and $Q(\xi)$ becomes locally- $L_{1}^{p}$. Using (19) and (10), we have (note that $\left.\operatorname{supp} \beta_{\gamma}^{\prime} \subset X_{\gamma}^{\prime \prime}\right)$

$$
\begin{aligned}
\left\|P_{A_{\gamma}}\left(\beta_{\gamma}^{\prime} \xi\right)\right\|_{L^{q}\left(X_{\gamma}^{\prime}, g\right)} & \leq\left\|P_{A_{\gamma}}\left(\beta_{\gamma}^{\prime} \xi\right)\right\|_{L^{q}\left(X_{\gamma}^{\prime}, g_{\gamma}\right)} \leq \operatorname{const}_{M}\left\|\beta_{\gamma}^{\prime} \xi\right\|_{L^{p}\left(X_{\gamma}, g_{\gamma}\right)} \\
& \leq \operatorname{const}_{M}\|\xi\|_{L^{p}\left(X_{\gamma}^{\prime \prime}, g_{\gamma}\right)} \leq \operatorname{const}_{M}^{\prime}\|\xi\|_{L^{p}\left(X_{\gamma}^{\prime \prime}, g\right)} .
\end{aligned}
$$

Therefore

$$
\|Q(\xi)\|_{B L^{q}} \leq \text { const }_{M} \cdot\|\xi\|_{B L^{p}}
$$

Let $\boldsymbol{A}^{\prime}=\boldsymbol{A}^{\prime}(\theta)$ be the connection on $X^{\sharp(\Gamma, S)}$ defined in Section 3.3. We have

$$
d_{\boldsymbol{A}^{\prime}}^{+} Q(\xi)=\sum_{\gamma \in \Gamma} d_{A_{\gamma}^{\prime}}^{+}\left(\beta_{\gamma} P_{A_{\gamma}}\left(\beta_{\gamma}^{\prime} \xi\right)\right)
$$

Since $d_{A_{\gamma}}^{+} P_{A_{\gamma}}=1$ and $\beta_{\gamma}^{\prime} \beta_{\gamma}=\beta_{\gamma}^{\prime}($ see $(17))$,

$$
\begin{aligned}
d_{A_{\gamma}^{\prime}}^{+}\left(\beta_{\gamma} P_{A_{\gamma}}\left(\beta_{\gamma}^{\prime} \xi\right)\right) & =\left(d \beta_{\gamma} \wedge P_{A_{\gamma}}\left(\beta_{\gamma}^{\prime} \xi\right)\right)^{+}+\beta_{\gamma} d_{A_{\gamma}^{\prime}}^{+} P_{A_{\gamma}}\left(\beta_{\gamma}^{\prime} \xi\right), \\
& =\beta_{\gamma}^{\prime} \xi+\left(d \beta_{\gamma} \wedge P_{A_{\gamma}}\left(\beta_{\gamma}^{\prime} \xi\right)\right)^{+}+\beta_{\gamma}\left[\left(A_{\gamma}^{\prime}-A_{\gamma}\right) \wedge P_{A_{\gamma}}\left(\beta_{\gamma}^{\prime} \xi\right)\right]^{+} .
\end{aligned}
$$

$\left\{\beta_{\gamma}^{\prime}\right\}$ is a partition of unity (see (18)). So

$$
d_{\boldsymbol{A}^{\prime}}^{+} Q(\xi)=\xi+\sum_{\gamma \in \Gamma}\left(d \beta_{\gamma} \wedge P_{A_{\gamma}}\left(\beta_{\gamma}^{\prime} \xi\right)\right)^{+}+\sum_{\gamma \in \Gamma} \beta_{\gamma}\left[\left(A_{\gamma}^{\prime}-A_{\gamma}\right) \wedge P_{A_{\gamma}}\left(\beta_{\gamma}^{\prime} \xi\right)\right]^{+}
$$


From Hölder's inequality $\left(L^{4} \times L^{q} \rightarrow L^{p}\right)$ and $(27)$

$$
\begin{aligned}
\|\left(d \beta_{\gamma}\right. & \left.\wedge P_{A_{\gamma}}\left(\beta_{\gamma}^{\prime} \xi\right)\right)^{+}\left\|_{L^{p}\left(X_{\gamma}^{\prime}, g\right)}+\right\|\left[\left(A_{\gamma}^{\prime}-A_{\gamma}\right) \wedge P_{A_{\gamma}}\left(\beta_{\gamma}^{\prime} \xi\right)\right]^{+} \|_{L^{p}\left(X_{\gamma}^{\prime}, g\right)} \\
& \leq \operatorname{const}_{M} \cdot\left(\left\|d \beta_{\gamma}\right\|_{L^{4}\left(X_{\gamma}, g\right)}+\left\|A_{\gamma}-A_{\gamma}^{\prime}\right\|_{L^{4}\left(X_{\gamma}, g\right)}\right)\|\xi\|_{L^{p}\left(X_{\gamma}^{\prime \prime}, g\right)} .
\end{aligned}
$$

Note that the $L^{4}$-norm of a 1-form is conformally invariant. So $\left\|d \beta_{\gamma}\right\|_{L^{4}\left(X_{\gamma}, g\right)}$ and $\left\|A_{\gamma}-A_{\gamma}^{\prime}\right\|_{L^{4}\left(X_{\gamma}, g\right)}$ are equal to $\left\|d \beta_{\gamma}\right\|_{L^{4}\left(X_{\gamma}, g_{\gamma}\right)}$ and $\left\|A_{\gamma}-A_{\gamma}^{\prime}\right\|_{L^{4}\left(X_{\gamma}, g_{\gamma}\right)}$, and these are very small (see (15) and (16)) for $N \gg 1$ and $b=4 N \sqrt{\lambda} \ll 1$. Then we get

$$
\left\|d_{\boldsymbol{A}^{\prime}}^{+} Q(\xi)-\xi\right\|_{B L^{p}} \leq \operatorname{const}_{M}\left((\log N)^{-3 / 4}+b^{2}\right)\|\xi\|_{B L^{p}} .
$$

Thus

Lemma 4.1. Set $R:=R_{\theta}:=d_{\boldsymbol{A}^{\prime}}^{+} Q-1: B L^{p} \rightarrow B L^{p}$. For any $\xi \in B L^{p}$

$$
\|R(\xi)\|_{B L^{p}} \leq \operatorname{const}_{M}\left((\log N)^{-3 / 4}+b^{2}\right)\|\xi\|_{B L^{p}} .
$$

Hence there exist positive constants $N_{0}=N_{0}(M)$ and $b_{0}=b_{0}(M)$ such that if $N \geq N_{0}$ and $b=4 N \sqrt{\lambda} \leq b_{0}$ then $\|R\| \leq 1 / 2$ and there exists $(1+R)^{-1}=\sum_{n \geq 0}(-R)^{n}: B L^{p} \rightarrow B L^{p}$. $P:=P_{\theta}:=Q(1+R)^{-1}$ gives a right inverse of $d_{\boldsymbol{A}^{\prime}}^{+}$:

$$
d_{\boldsymbol{A}^{\prime}}^{+} P(\xi)=\xi
$$

for any $\xi \in B L^{p}$. From (28), for any $\xi \in B L^{p}$

$$
\|P(\xi)\|_{B L^{q}} \leq \text { const }_{M} \cdot\|\xi\|_{B L^{p}} .
$$

We want to find an ASD connection of the form $\boldsymbol{A}^{\prime}+P(\xi)\left(\xi \in B L^{p}\right)$. Since $P$ is a right inverse of $d_{\boldsymbol{A}^{\prime}}^{+}$, this is equivalent to solving the following equation for $\xi \in B L^{p}$ :

$$
\xi+(P(\xi) \wedge P(\xi))^{+}=-F^{+}\left(\boldsymbol{A}^{\prime}\right) .
$$

From (7) we have $\operatorname{vol}\left(X_{\gamma}^{\prime \prime}, g\right) \leq 8^{4} \operatorname{vol}\left(X_{\gamma}^{\prime \prime}, g_{\gamma}\right) \leq 8^{4} \operatorname{vol}(X)$. From this and $q>4$, we have

$$
\sup _{\gamma \in \Gamma}\|P(\xi)\|_{L^{4}\left(X_{\gamma}^{\prime \prime}, g\right)} \leq \text { const } \cdot\|P(\xi)\|_{B L^{q}}
$$

where this "const" is a positive constant depending only on $\operatorname{vol}(X)$. Then, using Hölder's inequality $\left(L^{4} \times L^{q} \rightarrow L^{p}\right)$ and $(27)$, we get

$$
\left\|\left(P\left(\xi_{1}\right) \wedge P\left(\xi_{1}\right)\right)^{+}-\left(P\left(\xi_{2}\right) \wedge P\left(\xi_{2}\right)\right)^{+}\right\|_{B L^{p}} \leq \operatorname{const}_{M}\left(\left\|\xi_{1}\right\|_{B L^{p}}+\left\|\xi_{2}\right\|_{B L^{p}}\right)\left\|\xi_{1}-\xi_{2}\right\|_{B L^{p}} .
$$

Then we use the following lemma (this is [5, p. 289, Lemma (7.2.23)])

Lemma 4.2. Let $B$ be a Banach space and $k$ be a positive constant. Let $S: B \rightarrow B$ be a (not necessarily linear) map satisfying $S(0)=0$ and $\|S(x)-S(y)\| \leq k(\|x\|+\|y\|)\|x-y\|$. Then for any $y \in B$ with $\|y\| \leq 1 /(10 k)$ there uniquely exists $x \in B$ with $\|x\| \leq 1 /(5 k)$ satisfying

$$
x+S(x)=y .
$$

Moreover $x$ satisfies $\|x\| \leq\|y\|+2 k\|y\|^{2}$. 
Proof. Set $T(x):=y-S(x)$. It is easy to check that for $\|x\| \leq 1 /(5 k)$ we have $\|T(x)\| \leq$ $1 /(5 k)$ and for $\left\|x_{i}\right\| \leq 1 /(5 k)(i=1,2)$ we have $\left\|T\left(x_{1}\right)-T\left(x_{2}\right)\right\| \leq(2 / 5)\left\|x_{1}-x_{2}\right\|$. Then the contraction mapping principle implies that there uniquely exists $x$ with $\|x\| \leq 1 /(5 k)$ satisfying $T(x)=x$. If $x+S(x)=y$ and $\|x\| \leq 1 /(5 k)$, then $\|x\| \leq\|y\|+k\|x\|^{2} \leq$ $\|y\|+(1 / 5)\|x\|$. Hence $\|x\| \leq(5 / 4)\|y\|$. Therefore

$$
\|x-y\| \leq k\|x\|^{2} \leq(25 k / 16)\|y\|^{2} \leq 2 k\|y\|^{2} .
$$

From (15) we have

$$
\left\|F^{+}\left(\boldsymbol{A}^{\prime}\right)\right\|_{B L^{p}} \leq \operatorname{const}_{M} \cdot b^{4 / p} .
$$

Hence we can solve the equation (31) if $b \ll 1$.

Proposition 4.3. There are positive constants $N_{0}=N_{0}(M), b_{0}=b_{0}(M)^{1}, C_{1}=C_{1}(M)$ such that if $N \geq N_{0}$ and $b=4 N \sqrt{\lambda} \leq b_{0}$ then there exists $\boldsymbol{\xi}=\boldsymbol{\xi}(\theta) \in B L^{p}$ with $\|\boldsymbol{\xi}\|_{B L^{p}} \leq C_{1}$ satisfying

$$
F^{+}\left(\boldsymbol{A}^{\prime}+P(\boldsymbol{\xi})\right)=0 \quad \text { and } \quad\|\boldsymbol{\xi}\|_{B L^{p}} \leq \operatorname{const}_{M} \cdot b^{4 / p} .
$$

Moreover this $\boldsymbol{\xi}$ is unique, i.e., if $\eta \in B L^{p}$ with $\|\eta\|_{B L^{p}} \leq C_{1}$ satisfies $F^{+}\left(\boldsymbol{A}^{\prime}+P(\eta)\right)=0$ then $\eta=\boldsymbol{\xi}$. From (30),

$$
\|P(\boldsymbol{\xi})\|_{B L^{q}} \leq \text { const }_{M} \cdot b^{4 / p} .
$$

We will denote $\boldsymbol{A}(\theta):=\boldsymbol{A}^{\prime}+P(\boldsymbol{\xi})$.

Remark 4.4. We assume $2<p<4$. But the above construction argument is still true for $p=2$. In particular, we have

$$
\begin{array}{r}
\|\boldsymbol{\xi}\|_{B L^{2}}:=\sup _{\gamma \in \Gamma}\|\boldsymbol{\xi}\|_{L^{2}\left(X_{\gamma}^{\prime \prime}, g\right)} \leq \operatorname{const}_{M} \cdot b^{2}, \\
\|P(\boldsymbol{\xi})\|_{B L^{4}}:=\sup _{\gamma \in \Gamma}\|P(\boldsymbol{\xi})\|_{L^{4}\left(X_{\gamma}^{\prime \prime}, g\right)} \leq \operatorname{const}_{M} \cdot b^{2} .
\end{array}
$$

Remark 4.5. If $\left[E_{\gamma}, A_{\gamma}\right] \in M_{1}$ for all $\gamma \in \Gamma$, then $\boldsymbol{A}^{\prime}(\theta)$ is a flat connection (cf. Remark 3.3). In particular, $F^{+}\left(\boldsymbol{A}^{\prime}(\theta)\right)=0$. Hence we have $\boldsymbol{\xi}(\theta)=0$ and $\boldsymbol{A}(\theta)=\boldsymbol{A}^{\prime}(\theta)$.

4.2. Estimate on the curvature. We want to estimate the curvature of $\boldsymbol{A}=\boldsymbol{A}(\theta)$. From (15),

$$
\left\|F\left(\boldsymbol{A}^{\prime}\right)\right\|_{B L^{p}} \leq \sup _{\gamma \in \Gamma}\left\|F\left(A_{\gamma}\right)\right\|_{L^{p}\left(X_{\gamma}, g_{\gamma}\right)}+\operatorname{const}_{M} \cdot b^{4 / p} .
$$

For any $\xi \in B L^{p}$ we have

$$
d_{A^{\prime}} Q(\xi)=\sum_{\gamma} d \beta_{\gamma} \wedge P_{A_{\gamma}}\left(\beta_{\gamma}^{\prime} \xi\right)+\beta_{\gamma} d_{A_{\gamma}} P_{A_{\gamma}}\left(\beta_{\gamma}^{\prime} \xi\right)+\beta_{\gamma}\left[\left(A_{\gamma}^{\prime}-A_{\gamma}\right) \wedge P_{A_{\gamma}}\left(\beta_{\gamma}^{\prime} \xi\right)\right] .
$$

\footnotetext{
${ }^{1}$ This is an abuse of notation; these $N_{0}$ and $b_{0}$ are not necessarily equal to the constants in Lemma 4.1 .
} 
We can estimate this as in the previous subsection by using (19) and get:

$$
\left\|d_{A^{\prime}} Q(\xi)\right\|_{B L^{p}} \leq \operatorname{const}_{M}\|\xi\|_{B L^{p}} .
$$

Since $P=Q(1+R)^{-1}$, we have

$$
\left\|d_{\boldsymbol{A}^{\prime}} P(\xi)\right\|_{B L^{p}} \leq \operatorname{const}_{M}\left\|(1+R)^{-1} \xi\right\|_{B L^{p}} \leq \operatorname{const}_{M}^{\prime}\|\xi\| .
$$

We have $\boldsymbol{A}=\boldsymbol{A}^{\prime}+P(\boldsymbol{\xi})$ and $F(\boldsymbol{A})=F\left(\boldsymbol{A}^{\prime}\right)+d_{\boldsymbol{A}^{\prime}} P(\boldsymbol{\xi})+P(\boldsymbol{\xi}) \wedge P(\boldsymbol{\xi})$. Using $\|\boldsymbol{\xi}\|_{B L^{p}} \leq$ const $_{M} b^{4 / p}$, we get

$\|F(\boldsymbol{A})\|_{B L^{p}} \leq\left\|F\left(\boldsymbol{A}^{\prime}\right)\right\|_{B L^{p}}+\left\|d_{\boldsymbol{A}^{\prime}} P(\boldsymbol{\xi})\right\|_{B L^{p}}+$ const $\|P(\boldsymbol{\xi})\|_{B L^{q}}^{2} \leq\left\|F\left(\boldsymbol{A}^{\prime}\right)\right\|_{B L^{p}}+$ const $_{M} b^{4 / p}$.

Using (32) we get the conclusion:

Proposition 4.6. The ASD connection $\boldsymbol{A}(\theta)$ satisfies

$$
\|F(\boldsymbol{A}(\theta))\|_{B L^{p}} \leq \sup _{\gamma \in \Gamma}\left\|F\left(A_{\gamma}\right)\right\|_{L^{p}\left(X_{\gamma}, g_{\gamma}\right)}+\operatorname{const}_{M} \cdot b^{4 / p} .
$$

\section{INFINITE GLUING: INJECTIVITY PROBLEM}

Section 5 and Section 6 are technical. Some readers should skip these sections and go to Section 7, and return to them when the results in these sections are used. The main result in Section 5 is Proposition 5.5, and the main result in Section 6 is Theorem 6.11. They will be used later. Some arguments in Section 5.1, 6.2 and 6.3 (in particular, Corollary 6.8 and Lemma 6.10) will be also used later.

5.1. Variation. For each $\gamma \in \Gamma$, let $\theta:=\left(E_{\gamma}, A_{\gamma}, \rho_{\gamma, s}\right)_{\gamma \in \Gamma, s \in S}$ be a gluing data. Let $\alpha_{\gamma} \in H_{A_{\gamma}}^{1}$ with $\left\|\alpha_{\gamma}\right\|_{L^{q}} \leq \delta_{M}$ (see Section 3.4.2), and $\tilde{A}_{\gamma}:=A_{\gamma}+\tilde{\alpha}_{\gamma}$ be the ASD connection on $E_{\gamma}$ given in Section 3.4.2. Set $\boldsymbol{\alpha}:=\left(\alpha_{\gamma}\right)_{\gamma \in \Gamma}$. Let $v_{\gamma, s} \in\left(\operatorname{ad} E_{\gamma}\right)_{x_{\gamma, s}}(\gamma \in \Gamma, s \in S)$ with $\left|v_{\gamma, s}\right| \leq \operatorname{Diam}(S U(2))$. Set $\rho_{\gamma, s}^{\prime}:=\rho_{\gamma, s} e^{v_{\gamma, s}}$ and $\boldsymbol{v}:=\left(v_{\gamma, s}\right)_{\gamma \in \Gamma, s \in S}$. We define

$$
\|\boldsymbol{\alpha}\|:=\sup _{\gamma \in \Gamma}\left\|\alpha_{\gamma}\right\|_{L^{q}\left(X_{\gamma}, g_{\gamma}\right)}, \quad\|\boldsymbol{v}\|:=\sup _{\gamma \in \Gamma, s \in S}\left|v_{\gamma, s}\right| .
$$

Suppose $\left[E_{\gamma}, \tilde{A}_{\gamma}\right] \in M$ and set $\tilde{\theta}:=\left(E_{\gamma}, \tilde{A}_{\gamma}, \rho_{\gamma, s}^{\prime}\right)_{\gamma \in \Gamma, s \in S}$. We want to compare $\tilde{\boldsymbol{A}}:=\boldsymbol{A}(\tilde{\theta})$ with $\boldsymbol{A}:=\boldsymbol{A}(\theta)$. First we will construct a gauge transformation $h$ from $\tilde{\boldsymbol{E}}=\boldsymbol{E}(\tilde{\theta})$ to $\boldsymbol{E}=\boldsymbol{E}(\theta)$.

Let $u_{\gamma}:\left.E_{\gamma}\right|_{B\left(x_{\gamma, s}, b\right)} \rightarrow B\left(x_{\gamma, s}, b\right) \times\left(E_{\gamma}\right)_{x_{\gamma, s}}$ and $u_{\gamma}:\left.E_{\gamma}\right|_{B\left(y_{\gamma, s}, b\right)} \rightarrow B\left(y_{\gamma, s}, b\right) \times\left(E_{\gamma}\right)_{y_{\gamma, s}}$ be the exponential gauge of $A_{\gamma}$ around $x_{\gamma, s}$ and $y_{\gamma, s}(\gamma \in \Gamma, s \in S)$. We also denote $\tilde{u}_{\gamma}$ as the exponential gauge of $\tilde{A}_{\gamma}$ around $x_{\gamma, s}$ and $y_{\gamma, s}(\gamma \in \Gamma, s \in S)$. From (21) we have $\left|u_{\gamma}-\tilde{u}_{\gamma}\right| \leq \operatorname{const}_{M} \cdot b\|\boldsymbol{\alpha}\| \ll 1$. Hence there uniquely exists a section $w_{\gamma}$ of $\operatorname{ad} E_{\gamma}$ with $\left|w_{\gamma}\right| \leq$ const $_{M}^{\prime} \cdot b\|\boldsymbol{\alpha}\| \ll 1$ over $\bigsqcup_{s \in S} B\left(x_{\gamma, s}, b\right) \sqcup B\left(y_{\gamma, s}, b\right)$ satisfying $\tilde{u}_{\gamma}=u_{\gamma} e^{w_{\gamma}}$. We define a section $\hat{v}_{\gamma}$ of $\operatorname{ad} E_{\gamma}$ over $\bigsqcup_{s \in S} B\left(x_{\gamma, s}, b\right) \sqcup B\left(y_{\gamma, s}, b\right)$ by setting

$$
\hat{v}_{\gamma}:= \begin{cases}u_{\gamma}^{-1} \circ v_{\gamma, s} \circ u_{\gamma} & \text { on } B\left(x_{\gamma, s}, b\right)(s \in S), \\ -u_{\gamma}^{-1} \circ\left(\rho_{\gamma s^{-1}, s} \circ v_{\gamma s^{-1, s}} \circ \rho_{\gamma s^{-1, s}}^{-1}\right) \circ u_{\gamma} & \text { on } B\left(y_{\gamma, s}, b\right)(s \in S) .\end{cases}
$$


We define the gauge transformation $h_{\gamma}: E_{\gamma} \rightarrow E_{\gamma}$ by

$$
h_{\gamma}:= \begin{cases}e^{\left(1-\beta_{\gamma}^{\prime}\right) \hat{v}_{\gamma}} e^{\left(1-\psi_{\gamma}^{\prime}\right) w_{\gamma}} & \text { on } B\left(x_{\gamma, s}, b\right) \text { and } B\left(y_{\gamma, s}, b\right)(s \in S), \\ 1 & \text { otherwise, }\end{cases}
$$

where $\beta_{\gamma}^{\prime}$ and $\psi_{\gamma}^{\prime}$ are the cut-off functions introduced in Section 3.3. ( $\psi_{\gamma}^{\prime}$ satisfies $\left|d \psi_{\gamma}^{\prime}\right| \leq$ $8 / b, \psi_{\gamma}^{\prime}=0$ over $\bigsqcup_{s \in S} B\left(x_{s}, b / 4\right) \sqcup B\left(y_{s}, b / 4\right)$ and $\psi_{\gamma}^{\prime}=1$ over the complement of $\bigsqcup_{s \in S} B\left(x_{s}, b / 2\right) \sqcup B\left(y_{s}, b / 2\right)$.) Since $\beta_{\gamma}^{\prime}+\beta_{\gamma s}^{\prime}=1$ and $\psi_{\gamma}^{\prime}=0$ over $\Omega\left(x_{\gamma, s}\right)=\Omega\left(y_{\gamma s, s}\right)$ (remember: $\Omega\left(x_{\gamma, s}\right)=B\left(x_{\gamma, s}, N \sqrt{\lambda}\right) \backslash \bar{B}\left(x_{\gamma, s}, \sqrt{\lambda} / N\right)$ ), the diagram (35) becomes commutative.

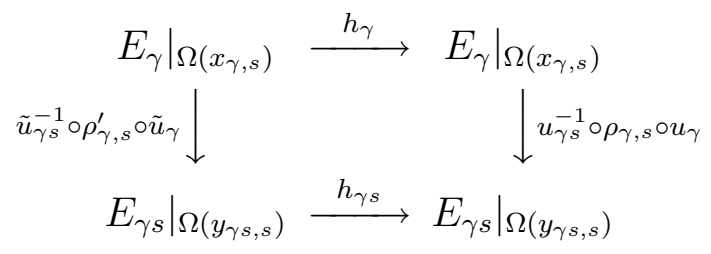

Therefore $\left\{h_{\gamma}\right\}$ compatibly define the gauge transformation $h=h_{\boldsymbol{v}, \boldsymbol{\alpha}}: \tilde{\boldsymbol{E}} \rightarrow \boldsymbol{E}$.

Set $P:=P_{\theta}$ and $P_{\boldsymbol{v}, \boldsymbol{\alpha}}:=h \circ P_{\tilde{\theta}} \circ h^{-1}: B L^{p} \rightarrow B L^{q}$. We set $\tilde{\boldsymbol{A}}^{\prime}:=\boldsymbol{A}^{\prime}(\tilde{\theta})$ (see Section $3.3)$ and

$$
\boldsymbol{A}_{\boldsymbol{v}, \boldsymbol{\alpha}}^{\prime}:=h\left(\tilde{\boldsymbol{A}}^{\prime}\right) \quad(\text { this is a connection on } \boldsymbol{E}) \text {. }
$$

Lemma 5.1. For any $\xi \in B L^{p}$,

$$
\left\|P_{\boldsymbol{v}, \boldsymbol{\alpha}}(\xi)-P(\xi)\right\|_{B L^{q}} \leq \operatorname{const}_{M}(\|\boldsymbol{\alpha}\|+\|\boldsymbol{v}\|)\|\xi\|_{B L^{p}} .
$$

Proof. The proof is just a confirmation of the definitions. We have

$$
P_{\boldsymbol{v}, \boldsymbol{\alpha}}=Q_{\boldsymbol{v}, \boldsymbol{\alpha}}\left(d_{\boldsymbol{A}_{\boldsymbol{v}, \boldsymbol{\alpha}}^{\prime}}^{+} Q_{\boldsymbol{v}, \boldsymbol{\alpha}}\right)^{-1}
$$

where

$$
\begin{aligned}
Q_{\boldsymbol{v}, \boldsymbol{\alpha}}(\xi) & =\sum_{\gamma \in \Gamma} h_{\gamma} \cdot \beta_{\gamma} P_{\tilde{A}_{\gamma}}\left(\beta_{\gamma}^{\prime} h_{\gamma}^{-1} \xi\right) \\
& =\sum_{\gamma \in \Gamma}\left(\left(h_{\gamma}-1\right) \beta_{\gamma} P_{\tilde{A}_{\gamma}}\left(\beta_{\gamma}^{\prime} h_{\gamma}^{-1}(\xi)\right)+\beta_{\gamma} P_{\tilde{A}_{\gamma}}\left(\beta_{\gamma}^{\prime}\left(h_{\gamma}^{-1}-1\right) \xi\right)+\beta_{\gamma} P_{\tilde{A}_{\gamma}}\left(\beta_{\gamma}^{\prime} \xi\right)\right) .
\end{aligned}
$$

By using the definition (34) and Lemma 3.6, we get

$$
\left\|Q_{\boldsymbol{v}, \boldsymbol{\alpha}}(\xi)-Q(\xi)\right\|_{B L^{q}} \leq \operatorname{const}_{M}(\|\boldsymbol{\alpha}\|+\|\boldsymbol{v}\|)\|\xi\|_{B L^{p}} .
$$

In a similar way (cf. (29)),

$$
\left\|\left(d_{\boldsymbol{A}_{\boldsymbol{v}, \boldsymbol{\alpha}}^{\prime}}^{+} Q_{\boldsymbol{v}, \boldsymbol{\alpha}}\right)^{-1}(\xi)-\left(d_{\boldsymbol{A}^{\prime}}^{+} Q\right)^{-1}(\xi)\right\|_{B L^{p}} \leq \operatorname{const}_{M}(\|\boldsymbol{\alpha}\|+\|\boldsymbol{v}\|)\|\xi\|_{B L^{p}} .
$$

Then we get the above conclusion.

Set $\boldsymbol{\xi}_{\boldsymbol{v}, \boldsymbol{\alpha}}:=h(\boldsymbol{\xi}(\tilde{\theta})) \in B L^{p}\left(\Omega^{+}(\operatorname{ad} \boldsymbol{E})\right)$. We have $\left\|\boldsymbol{\xi}_{\boldsymbol{v}, \boldsymbol{\alpha}}\right\|_{B L^{p}} \leq \operatorname{const}_{M} \cdot b^{4 / p}$ (see Proposition 4.3). 


\section{Lemma 5.2.}

$$
\left\|\boldsymbol{\xi}_{\boldsymbol{v}, \boldsymbol{\alpha}}-\boldsymbol{\xi}\right\|_{B L^{p}} \leq \operatorname{const}_{M} \cdot b^{4 / p} \cdot\left(\|\boldsymbol{\alpha}\|+b^{2}\|\boldsymbol{v}\|\right) .
$$

Proof. We have (see (31) and Proposition 4.3)

$$
\boldsymbol{\xi}_{\boldsymbol{v}, \boldsymbol{\alpha}}+\left(P_{\boldsymbol{v}, \boldsymbol{\alpha}}\left(\boldsymbol{\xi}_{\boldsymbol{v}, \boldsymbol{\alpha}}\right) \wedge P_{\boldsymbol{v}, \boldsymbol{\alpha}}\left(\boldsymbol{\xi}_{\boldsymbol{v}, \boldsymbol{\alpha}}\right)\right)^{+}=-F^{+}\left(\boldsymbol{A}_{\boldsymbol{v}, \boldsymbol{\alpha}}^{\prime}\right), \quad \boldsymbol{\xi}+(P(\boldsymbol{\xi}) \wedge P(\boldsymbol{\xi}))^{+}=-F^{+}\left(\boldsymbol{A}^{\prime}\right) .
$$

By using (21), Lemma 3.9 and $h_{\gamma}=1$ in the support of $F^{+}\left(\tilde{A}_{\gamma}^{\prime}\right)$ (cf. Section 3.3), we have

$$
\left\|F^{+}\left(\boldsymbol{A}_{\boldsymbol{v}, \boldsymbol{\alpha}}^{\prime}\right)-F^{+}\left(\boldsymbol{A}^{\prime}\right)\right\|_{B L^{p}} \leq \operatorname{const}_{M} \cdot b^{4 / p}\|\boldsymbol{\alpha}\| .
$$

From Proposition 4.3, Remark 4.4, Lemma 5.1 and Hölder's inequality $B L^{4} \times B L^{q} \rightarrow B L^{p}$,

$$
\begin{array}{r}
\left\|\left(P_{\boldsymbol{v}, \boldsymbol{\alpha}}\left(\boldsymbol{\xi}_{\boldsymbol{v}, \boldsymbol{\alpha}}\right) \wedge P_{\boldsymbol{v}, \boldsymbol{\alpha}}\left(\boldsymbol{\xi}_{\boldsymbol{v}, \boldsymbol{\alpha}}\right)\right)^{+}-(P(\boldsymbol{\xi}) \wedge P(\boldsymbol{\xi}))^{+}\right\|_{B L^{p}}, \\
\leq \text { const }_{M} \cdot b^{2}\left\|\boldsymbol{\xi}_{\boldsymbol{v}, \boldsymbol{\alpha}}-\boldsymbol{\xi}\right\|_{B L^{p}}+\text { const }_{M} \cdot b^{2+4 / p}(\|\boldsymbol{\alpha}\|+\|\boldsymbol{v}\|) .
\end{array}
$$

Hence

$$
\left\|\boldsymbol{\xi}_{\boldsymbol{v}, \boldsymbol{\alpha}}-\boldsymbol{\xi}\right\|_{B L^{p}} \leq \operatorname{const}_{M} \cdot b^{2}\left\|\boldsymbol{\xi}_{\boldsymbol{v}, \boldsymbol{\alpha}}-\boldsymbol{\xi}\right\|_{B L^{p}}+\operatorname{const}_{M} \cdot b^{4 / p}\left(\|\boldsymbol{\alpha}\|+b^{2}\|\boldsymbol{v}\|\right)
$$

Since $b \ll 1$, we get the desired estimate.

\section{Corollary 5.3.}

$$
\left\|P_{\boldsymbol{v}, \boldsymbol{\alpha}}\left(\boldsymbol{\xi}_{\boldsymbol{v}, \boldsymbol{\alpha}}\right)-P(\boldsymbol{\xi})\right\|_{B L^{q}} \leq \text { const }_{M} \cdot b^{4 / p}(\|\boldsymbol{\alpha}\|+\|\boldsymbol{v}\|) .
$$

Set $a_{\gamma}:=\left.P(\boldsymbol{\xi})\right|_{X_{\gamma}^{\prime \prime}} \in \Omega_{X_{\gamma}^{\prime \prime}}^{1}\left(\operatorname{ad} E_{\gamma}\right)$ and $\tilde{a}_{\gamma}:=\left.P_{\tilde{\theta}}\left(\boldsymbol{\xi}_{\tilde{\theta}}\right)\right|_{X_{\gamma}^{\prime \prime}}=\left.h_{\gamma}^{-1}\left(P_{\boldsymbol{v}, \boldsymbol{\alpha}}\left(\boldsymbol{\xi}_{\boldsymbol{v}, \boldsymbol{\alpha}}\right)\right)\right|_{X_{\gamma}^{\prime \prime}} \in \Omega_{X_{\gamma}^{\prime \prime}}^{1}\left(\operatorname{ad} E_{\gamma}\right)$ for each $\gamma \in \Gamma$. We have $\left.\boldsymbol{A}\right|_{X_{\gamma}^{\prime \prime}}=A_{\gamma}^{\prime}+a_{\gamma}$ and $\left.\tilde{\boldsymbol{A}}\right|_{X_{\gamma}^{\prime \prime}}=\tilde{A}_{\gamma}^{\prime}+\tilde{a}_{\gamma}$.

Lemma 5.4 .

$$
\sup _{\gamma \in \Gamma}\left\|a_{\gamma}-\tilde{a}_{\gamma}\right\|_{L^{q}\left(X_{\gamma}^{\prime \prime}, g_{\gamma}\right)} \leq \operatorname{const}_{M} \cdot b^{4 / p}(\|\boldsymbol{\alpha}\|+\|\boldsymbol{v}\|)
$$

Proof.

$$
\tilde{a}_{\gamma}-a_{\gamma}=\left(h_{\gamma}^{-1}-1\right) P_{\boldsymbol{v}, \boldsymbol{\alpha}}\left(\boldsymbol{\xi}_{\boldsymbol{v}, \boldsymbol{\alpha}}\right) h_{\gamma}+P_{\boldsymbol{v}, \boldsymbol{\alpha}}\left(\boldsymbol{\xi}_{\boldsymbol{v}, \boldsymbol{\alpha}}\right)\left(h_{\gamma}-1\right)+\left(P_{\boldsymbol{v}, \boldsymbol{\alpha}}\left(\boldsymbol{\xi}_{\boldsymbol{v}, \boldsymbol{\alpha}}\right)-P(\boldsymbol{\xi})\right) .
$$

From Proposition 4.3 and Corollary 5.3, we get the above estimate.

5.2. Injectivity problem. The purpose of this subsection is to prove the following.

Proposition 5.5. There exists $N_{0}=N_{0}(M)$ and $b_{0}=b_{0}(M)$ such that if $N \geq N_{0}$ and $b=4 N \sqrt{\lambda} \leq b_{0}$ then the following holds: Let $\theta=\left(E_{\gamma}, A_{\gamma}, \rho_{\gamma, s}\right)_{\gamma \in \Gamma, s \in S}$ and $\theta^{\prime}=$ $\left(F_{\gamma}, B_{\gamma}, \rho_{\gamma, s}^{\prime}\right)_{\gamma \in \Gamma, s \in S}$ be two gluing data. Then $\boldsymbol{A}(\theta)$ is gauge equivalent to $\boldsymbol{A}\left(\theta^{\prime}\right)$ if and only if $[\theta]=\left[\theta^{\prime}\right]$ in GID.

Proof. The "if" part is a direct consequence of the definitions. So we will give the proof of the "only if" part. We set $\boldsymbol{A}_{1}:=\boldsymbol{A}(\theta)$ and $\boldsymbol{A}_{2}:=\boldsymbol{A}\left(\theta^{\prime}\right)$. Suppose there is a gauge 
transformation $g: \boldsymbol{E}(\theta) \rightarrow \boldsymbol{E}\left(\theta^{\prime}\right)$ satisfying $g\left(\boldsymbol{A}_{1}\right)=\boldsymbol{A}_{2}$. We define $X_{\gamma, b}(\gamma \in \Gamma)$ as the complement of the $b$-balls $\bar{B}\left(x_{\gamma, s}, b\right)$ and $\bar{B}\left(y_{\gamma, s}, b\right)$ in $X_{\gamma}$ :

$$
X_{\gamma, b}:=X_{\gamma} \backslash\left(\bigcup_{s \in S} \bar{B}\left(x_{\gamma, s}, b\right) \cup \bar{B}\left(y_{\gamma, s}, b\right)\right) .
$$

By the definitions of the cut-off functions in Section 3.3, we have $A_{\gamma}^{\prime}=A_{\gamma}$ and $B_{\gamma}^{\prime}=B_{\gamma}$ over $X_{\gamma, b}$. From Proposition 4.3, we have

$$
\left\|A_{\gamma}-\boldsymbol{A}_{1}\right\|_{L^{q}\left(X_{\gamma, b}, g_{\gamma}\right)},\left\|B_{\gamma}-\boldsymbol{A}_{2}\right\|_{L^{q}\left(X_{\gamma, b}, g_{\gamma}\right)} \leq \operatorname{const}_{M} \cdot b^{4 / p} .
$$

Since $\boldsymbol{A}_{1}$ is gauge equivalent to $\boldsymbol{A}_{2}$,

$$
d_{L^{q}}\left(\left[\left.A_{\gamma}\right|_{X_{\gamma, b}}\right],\left[\left.B_{\gamma}\right|_{X_{\gamma, b}}\right]\right) \leq \operatorname{const}_{M} \cdot b^{4 / p} \ll 1 .
$$

From Lemma $3.7(2)$, this implies (for $b \ll 1$ ) $E_{\gamma} \cong F_{\gamma}$ for all $\gamma \in \Gamma$. Moreover, from Lemma 3.7 (1), Lemma 3.5 and the inequality (20), there exists $\alpha_{\gamma} \in H_{A_{\gamma}}^{1}$ with $\left\|\alpha_{\gamma}\right\|_{L^{q}} \leq$ const $_{M} \cdot b^{4 / p}$ for each $\gamma \in \Gamma$ such that $B_{\gamma}$ is gauge equivalent to $\tilde{A}_{\gamma}:=A_{\gamma}+\tilde{\alpha}_{\gamma}$. We can suppose $B_{\gamma}=\tilde{A}_{\gamma}$ without loss of generality.

$\rho_{\gamma, s}$ and $\rho_{\gamma, s}^{\prime}$ are $S U(2)$-isomorphisms between $\left(E_{\gamma}\right)_{x_{\gamma, s}}$ and $\left(E_{\gamma s}\right)_{y_{\gamma s, s}}(\gamma \in \Gamma, s \in S)$. Take $v_{\gamma, s} \in(\operatorname{ad} E)_{x_{\gamma, s}}$ such that $\rho_{\gamma, s}^{\prime}=\rho_{\gamma, s} e^{v_{\gamma, s}}$ and $\left|v_{\gamma, s}\right|=d\left(\rho_{\gamma, s}, \rho_{\gamma, s}^{\prime}\right)(\leq \operatorname{Diam}(S U(2)))$. Set $\boldsymbol{\alpha}:=\left(\alpha_{\gamma}\right)_{\gamma \in \Gamma}$ and $\boldsymbol{v}:=\left(v_{\gamma, s}\right)_{\gamma \in \Gamma, s \in S}$ as in Section 5.1. From the assumption, there are gauge transformations $g_{\gamma}$ of $E_{\gamma}$ over $X_{\gamma}^{\prime \prime}$ such that

$$
g_{\gamma}\left(A_{\gamma}^{\prime}+a_{\gamma}\right)=\tilde{A}_{\gamma}^{\prime}+\tilde{a}_{\gamma} \text { over } X_{\gamma}^{\prime \prime}
$$

where $a_{\gamma}$ and $\tilde{a}_{\gamma}$ are the element of $\Omega_{X_{\gamma}^{\prime \prime}}^{1}\left(\operatorname{ad} E_{\gamma}\right)$ satisfying $\boldsymbol{A}(\theta)=A_{\gamma}^{\prime}+a_{\gamma}$ and $\boldsymbol{A}\left(\theta^{\prime}\right)=\tilde{A}_{\gamma}^{\prime}+$ $\tilde{a}_{\gamma}$ over $X_{\gamma}^{\prime \prime}$ as in Section 5.1. Moreover $g_{\gamma}$ satisfy the following compatibility condition:

$$
\rho_{\gamma, s}^{\prime} \circ g_{\gamma}=g_{\gamma s} \circ \rho_{\gamma, s} \quad \text { over } \Omega\left(x_{\gamma, s}\right)=\Omega\left(y_{\gamma s, s}\right) .
$$

Let $I_{A_{\gamma}}$ be the isotropy group of $A_{\gamma}$. From Lemma 3.8, we have

$$
\min _{h \in I_{A_{\gamma}}}\left\|g_{\gamma}-h\right\|_{\mathcal{C}^{0}\left(X_{\gamma}^{\prime \prime}\right)} \leq \text { const }_{M}\left\|d_{A_{\gamma}^{\prime}} g_{\gamma}\right\|_{L^{q}\left(X_{\gamma}^{\prime \prime}, g_{\gamma}\right)},
$$

Using the action of $\prod_{\gamma \in \Gamma} I_{A_{\gamma}}$ on the gluing data, we can assume that

$$
\left\|g_{\gamma}-1\right\|_{\mathcal{C}^{0}\left(X_{\gamma}^{\prime \prime}\right)} \leq \operatorname{const}_{M}\left\|d_{A_{\gamma}^{\prime}} g_{\gamma}\right\|_{L^{q}\left(X_{\gamma}^{\prime \prime}, g_{\gamma}\right)} .
$$

From (38),

$$
\left\|d_{A_{\gamma}^{\prime}} g_{\gamma}\right\|_{L^{q}\left(X_{\gamma}^{\prime \prime}\right)} \leq\left\|A_{\gamma}^{\prime}-\tilde{A}_{\gamma}^{\prime}\right\|_{L^{q}\left(X_{\gamma}^{\prime \prime}\right)}+2\left\|g_{\gamma}-1\right\|_{\mathcal{C}^{0}\left(X_{\gamma}^{\prime \prime}\right)}\left\|a_{\gamma}\right\|_{L^{q}\left(X_{\gamma}^{\prime \prime}\right)}+\left\|\tilde{a}_{\gamma}-a_{\gamma}\right\|_{L^{q}\left(X_{\gamma}^{\prime \prime}\right)} .
$$

Using Lemma 3.9, we get $\left\|A_{\gamma}^{\prime}-\tilde{A}_{\gamma}^{\prime}\right\|_{L^{q}\left(X_{\gamma}^{\prime \prime}\right)} \leq \operatorname{const}_{M} \cdot\left\|\alpha_{\gamma}\right\|_{L^{q}\left(X_{\gamma}\right)}$. From Proposition 4.3 and Lemma 5.4, we get $\left\|a_{\gamma}\right\|_{L^{q}\left(X_{\gamma}^{\prime \prime}\right)} \leq \operatorname{const}_{M} \cdot b^{4 / p} \ll 1$ and $\left\|a_{\gamma}-\tilde{a}_{\gamma}\right\|_{L^{q}\left(X_{\gamma}^{\prime \prime}\right)} \leq$ const $_{M}$. $b^{4 / p}(\|\boldsymbol{\alpha}\|+\|\boldsymbol{v}\|)$. Therefore (using (40))

$$
\left\|g_{\gamma}-1\right\|_{\mathcal{C}^{0}\left(X_{\gamma}^{\prime \prime}\right)} \leq \operatorname{const}_{M}\|\boldsymbol{\alpha}\|+\operatorname{const}_{M} \cdot b^{4 / p}\|\boldsymbol{v}\| .
$$


On the other hand, from (38), $g_{\gamma}\left(A_{\gamma}\right)-\tilde{A}_{\gamma}=\left(\tilde{a}_{\gamma}-a_{\gamma}\right)+\left(1-g_{\gamma}\right) a_{\gamma}+g_{\gamma} a_{\gamma}\left(1-g_{\gamma}^{-1}\right)$ over $X_{\gamma, b}$ where $A_{\gamma}^{\prime}=A_{\gamma}$ and $\tilde{A}_{\gamma}^{\prime}=\tilde{A}_{\gamma}$. Hence (using (20) and Lemma 3.7 (1))

$\left\|\alpha_{\gamma}\right\| \leq \operatorname{const}_{M} \cdot d_{L^{q}}\left(\left[\left.A_{\gamma}\right|_{X_{\gamma, b}}\right],\left[\left.\tilde{A}_{\gamma}\right|_{X_{\gamma, b}}\right]\right) \leq \operatorname{const}_{M}\left(\left\|a_{\gamma}-\tilde{a}_{\gamma}\right\|_{L^{q}\left(X_{\gamma}^{\prime \prime}\right)}+\left\|g_{\gamma}-1\right\|_{\mathcal{C}^{0}\left(X_{\gamma}^{\prime \prime}\right)}\left\|a_{\gamma}\right\|_{L^{q}\left(X_{\gamma}\right)}\right)$

Using (41), Proposition 4.3 and Lemma 5.4,

$$
\|\boldsymbol{\alpha}\| \leq \operatorname{const}_{M} \cdot b^{4 / p}(\|\boldsymbol{\alpha}\|+\|\boldsymbol{v}\|)
$$

Since $b \ll 1$, we get

$$
\|\boldsymbol{\alpha}\| \leq \operatorname{const}_{M} \cdot b^{4 / p}\|\boldsymbol{v}\| .
$$

Substituting this into (41), we get $\left\|g_{\gamma}-1\right\|_{\mathcal{C}^{0}\left(X_{\gamma}^{\prime \prime}\right)} \leq \operatorname{const}_{M} \cdot b^{4 / p}\|\boldsymbol{v}\|$.

From the compatibility condition (39), $\rho_{\gamma, s}^{\prime}-\rho_{\gamma, s}=\left(g_{\gamma s}-1\right) \rho_{\gamma, s} g_{\gamma}+\rho_{\gamma, s}\left(g_{\gamma}^{-1}-1\right)$. Hence

$$
\left|\rho_{\gamma, s}^{\prime}-\rho_{\gamma, s}\right| \leq\left\|g_{\gamma s}-1\right\|_{\mathcal{C}^{0}\left(X_{\gamma}^{\prime \prime}\right)}+\left\|g_{\gamma}-1\right\|_{\mathcal{C}^{0}\left(X_{\gamma}^{\prime \prime}\right)} \leq \operatorname{const}_{M} \cdot b^{4 / p}\|\boldsymbol{v}\|
$$

Then

$$
\|\boldsymbol{v}\| \leq \mathrm{const} \cdot \sup _{\gamma, s}\left|\rho_{\gamma, s}^{\prime}-\rho_{\gamma, s}\right| \leq \operatorname{const}_{M} \cdot b^{4 / p}\|\boldsymbol{v}\| .
$$

Since $b \ll 1$, we get $\|\boldsymbol{v}\|=0$. This implies $\rho^{\prime}=\rho$ and (using (42)) $\|\boldsymbol{\alpha}\|=0$. Therefore $B_{\gamma}=\tilde{A}_{\gamma}=A_{\gamma}$.

\section{INFINITE GLUING: SURJECTIVITY PROBLEM}

In this section we will study a "surjectivity problem". We basically follow the argument of Donaldson-Kronheimer [5, Section 7.2.4, 7.2.5]. But our case is more involved because we cannot use the usual index-theorem argument. (This difficulty is suggested in $[5, \mathrm{p}$. 298].)

6.1. Linearized problem. Let $\theta=\left(E_{\gamma}, A_{\gamma}, \rho_{\gamma, s}\right)_{\gamma \in \Gamma, s \in S}$ be a gluing data. We denote $I$ as the set of $\gamma \in \Gamma$ satisfying $\left[E_{\gamma}, A_{\gamma}\right] \in M_{1}$. If $M_{1}=\emptyset$, then $I=\emptyset$. Set $\boldsymbol{E}:=\boldsymbol{E}(\theta)$ and $\boldsymbol{A}^{\prime}:=\boldsymbol{A}^{\prime}(\theta)$. We define $\boldsymbol{V}$ and $\boldsymbol{H}$ by

$$
\begin{aligned}
& \boldsymbol{V}:=\left\{\boldsymbol{v}=\left(v_{\gamma, s}\right)_{\gamma \in \Gamma, s \in S} \in \prod_{\gamma \in \Gamma, s \in S}\left(\operatorname{ad} E_{\gamma}\right)_{x_{\gamma, s}}\left|\|\boldsymbol{v}\|:=\sup _{\gamma, s}\right| v_{\gamma, s} \mid<\infty\right\}, \\
& \boldsymbol{H}:=\left\{\boldsymbol{\alpha}=\left(\alpha_{\gamma}\right)_{\gamma \in \Gamma} \in \prod_{\gamma \in \Gamma} H_{A_{\gamma}}^{1} \mid\|\boldsymbol{\alpha}\|:=\sup _{\gamma}\left\|\alpha_{\gamma}\right\|_{L^{q}\left(X_{\gamma}, g_{\gamma}\right)}<\infty\right\} .
\end{aligned}
$$

Note that $H_{A_{\gamma}}^{1}=0$ for $\left[E_{\gamma}, A_{\gamma}\right] \in M_{1}$. For $\boldsymbol{v} \in \boldsymbol{V}$ and $\boldsymbol{\alpha} \in \boldsymbol{H}$, we define $j_{1}(\boldsymbol{v})$ and $j_{2}(\boldsymbol{\alpha})$ in $\Omega^{1}(\operatorname{ad} \boldsymbol{E})$ by

$$
j_{1}(\boldsymbol{v}):=\left.\frac{\partial}{\partial t}\right|_{t=0} \boldsymbol{A}_{t \boldsymbol{v}, 0}^{\prime}, \quad j_{2}(\boldsymbol{\alpha}):=\left.\frac{\partial}{\partial t}\right|_{t=0} \boldsymbol{A}_{0, t \boldsymbol{\alpha}}^{\prime},
$$

where $\boldsymbol{A}_{\boldsymbol{v}, \boldsymbol{\alpha}}^{\prime}$ denotes a connection on $\boldsymbol{E}$ defined as in (36).

$\rho_{\gamma, s}$ is a $S U(2)$-isomorphism between the fibers $\left(E_{\gamma}\right)_{x_{\gamma, s}}$ and $\left(E_{\gamma s}\right)_{y_{\gamma s, s}}$. In this subsection, we identify the fibers $\left(E_{\gamma}\right)_{x_{\gamma, s}}$ and $\left(E_{\gamma s}\right)_{y_{\gamma, s}}$ by the given $\rho_{\gamma, s}$. Let $\boldsymbol{v}=\left(v_{\gamma, s}\right)_{\gamma, s}$ 
where $v_{\gamma, s} \in\left(\operatorname{ad} E_{\gamma}\right)_{x_{\gamma, s}} \cong\left(\operatorname{ad} E_{\gamma s}\right)_{y_{\gamma s, s}}$. We often consider $v_{\gamma, s}$ as a section of $\operatorname{ad} E_{\gamma}$ (or $\operatorname{ad} E_{\gamma s}$ ) over $\Omega\left(x_{\gamma, s}\right)$ (or $\Omega\left(y_{\gamma s, s}\right)$ ) by using the exponential gauge of $A_{\gamma}$ (or $A_{\gamma s}$ ): $\left.E_{\gamma}\right|_{\Omega\left(x_{\gamma, s}\right)} \cong \Omega\left(x_{\gamma, s}\right) \times\left(E_{\gamma}\right)_{x_{\gamma, s}}\left(\right.$ or $\left.\left.E_{\gamma s}\right|_{\Omega\left(y_{\gamma s, s}\right)} \cong \Omega\left(y_{\gamma s, s}\right) \times\left(E_{\gamma s}\right)_{y_{\gamma s, s}}\right)$. Then the above $j_{1}(\boldsymbol{v})$ is expressed by

$$
j_{1}(\boldsymbol{v})= \begin{cases}d_{A_{\gamma}^{\prime}}\left(\beta_{\gamma}^{\prime} v_{\gamma s}\right)=d \beta_{\gamma}^{\prime} \otimes v_{\gamma, s} & \text { over } \Omega\left(x_{\gamma, s}\right) \\ -d_{A_{\gamma}^{\prime}}\left(\beta_{\gamma}^{\prime} v_{\gamma s^{-1}, s}\right)=-d \beta_{\gamma}^{\prime} \otimes v_{\gamma s^{-1}, s} & \text { over } \Omega\left(y_{\gamma, s}\right),\end{cases}
$$

and we have $\operatorname{supp}\left(j_{1}(\boldsymbol{v})\right) \subset \bigcup_{\gamma} \operatorname{supp}\left(d \beta_{\gamma}^{\prime}\right) \subset \bigcup_{\gamma, s}\left(\Omega\left(x_{\gamma, s}\right) \cup \Omega\left(y_{\gamma, s}\right)\right)$. From this we easily deduce that $d_{\boldsymbol{A}^{\prime}}^{+} j_{1}(\boldsymbol{v})=0$.

\section{Lemma 6.1.}

$$
\left\|j_{2}(\boldsymbol{\alpha})-\sum_{\gamma \in \Gamma} \beta_{\gamma}^{\prime} \alpha_{\gamma}\right\|_{B L^{q}} \leq \operatorname{const}_{M} \cdot b^{4 / q}\|\boldsymbol{\alpha}\| .
$$

In particular, $\left\|j_{2}(\boldsymbol{\alpha})\right\|_{B L^{q}} \leq$ const $_{M}\|\boldsymbol{\alpha}\|$. Moreover

$$
\left\|d_{\boldsymbol{A}^{\prime}}^{+} j_{2}(\boldsymbol{\alpha})\right\|_{B L^{p}} \leq \operatorname{const}_{M} \cdot b^{4 / p}\|\boldsymbol{\alpha}\|
$$

Proof. We have $j_{2}(\boldsymbol{\alpha})=\alpha_{\gamma}$ over $X_{\gamma} \backslash \bigcup_{s}\left(B\left(x_{\gamma, s}, b\right) \cup B\left(y_{\gamma, s}, b\right)\right)$. Using Lemma 3.10, we have

$$
\left|j_{2}(\alpha)\right|_{g_{\gamma}} \leq \text { const }_{M}\|\boldsymbol{\alpha}\| \quad \text { over } \bigcup_{s}\left(B\left(x_{\gamma, s}, b\right) \cup B\left(y_{\gamma, s}, b\right)\right) .
$$

Therefore we get the first inequality. We have $d_{\boldsymbol{A}^{\prime}}^{+} j_{2}(\boldsymbol{\alpha})=0$ over $X_{\gamma} \backslash \bigcup_{s}\left(B\left(x_{\gamma, s}, b\right) \cup\right.$ $\left.B\left(y_{\gamma, s}, b\right)\right)$, where $F^{+}\left(\boldsymbol{A}_{0, t \boldsymbol{\alpha}}\right)=0$. Since $d_{\boldsymbol{A}^{\prime}}^{+} j_{2}(\boldsymbol{\alpha})=\partial F^{+}\left(\boldsymbol{A}_{0, t \boldsymbol{\alpha}}^{\prime}\right) /\left.\partial t\right|_{t=0}$ and $h_{\gamma}=1$ in the support of $F^{+}\left(\boldsymbol{A}_{0, t \boldsymbol{\alpha}}^{\prime}\right)$, the estimate (24) gives

$$
\left|d_{\boldsymbol{A}^{\prime}}^{+} j_{2}(\boldsymbol{\alpha})\right| \leq \text { const }_{M}\|\boldsymbol{\alpha}\| \quad \text { over } \bigcup_{\gamma, s}\left(B\left(x_{\gamma, s}, b\right) \cup B\left(y_{\gamma, s}, b\right)\right) .
$$

Therefore $\left\|d_{\boldsymbol{A}^{\prime}}^{+} j_{2}(\boldsymbol{\alpha})\right\|_{B L^{p}} \leq \operatorname{const}_{M} \cdot b^{4 / p}\|\boldsymbol{\alpha}\|$.

Fix $z \in X \backslash\left\{x_{s}, y_{s} \mid s \in S\right\}$. We take $b=4 N \sqrt{\lambda}>0$ so small that the balls of radius $b$ around $x_{s}$ and $y_{s}(s \in S)$ don't contain $z$. Let $z_{\gamma}(\gamma \in \Gamma)$ be the point in $X_{\gamma}$ corresponding to $z$. We define $\Omega(\operatorname{ad} \boldsymbol{E})_{0}$ as the set of $\chi \in \Omega(\operatorname{ad} \boldsymbol{E})$ satisfying $\chi\left(z_{\gamma}\right)=0$ for all $\gamma \in I$ (i.e., $\left.\left[E_{\gamma}, A_{\gamma}\right] \in M_{1}\right)$. Here we consider $z_{\gamma} \in X_{\gamma}^{\prime \prime} \subset X^{\sharp(\Gamma, S)}$.

Lemma 6.2. For any $\chi \in \Omega^{0}(\operatorname{ad} \boldsymbol{E})_{0}, \boldsymbol{v} \in \boldsymbol{V}$ and $\boldsymbol{\alpha} \in \boldsymbol{H}$,

$$
\|\chi\|_{\mathcal{C}^{0}}+\|\boldsymbol{v}\|+\|\boldsymbol{\alpha}\| \leq \operatorname{const}_{M}\left\|d_{\boldsymbol{A}^{\prime}} \chi+j_{1}(\boldsymbol{v})+j_{2}(\boldsymbol{\alpha})\right\|_{B L^{q}} .
$$

Proof. For each $\gamma \in \Gamma$ we define a section $\chi_{\gamma}$ of $\operatorname{ad} E_{\gamma}$ over $X_{\gamma}^{\prime \prime}$ by

$$
\chi_{\gamma}:=\chi+\sum_{s}\left(\beta_{\gamma}^{\prime}-1\right) v_{\gamma, s}+\sum_{s}\left(1-\beta_{\gamma}^{\prime}\right) v_{\gamma s^{-1}, s} .
$$


We have $d_{A_{\gamma}^{\prime}} \chi_{\gamma}=d_{\boldsymbol{A}^{\prime}} \chi+j_{1}(\boldsymbol{v})$ over $X_{\gamma}^{\prime \prime}$ and $\chi_{\gamma s}-\chi_{\gamma}=v_{\gamma, s}$ over $X_{\gamma}^{\prime \prime} \cap X_{\gamma s}^{\prime \prime}$. We have $\|\chi\|_{\mathcal{C}^{0}}+\|\boldsymbol{v}\| \leq(4|S|+3) \sup _{\gamma}\left\|\chi_{\gamma}\right\|_{\mathcal{C}^{0}\left(X_{\gamma}^{\prime \prime}\right)}$. If $\gamma \in I$, then $\chi_{\gamma}\left(z_{\gamma}\right)=0$. If $\gamma \notin I$, then $A_{\gamma}$ is irreducible. Therefore

$$
\begin{aligned}
\left\|\chi_{\gamma}\right\|_{\mathcal{C}^{0}\left(X_{\gamma}^{\prime \prime}\right)}+\left\|\alpha_{\gamma}\right\|_{L^{q}\left(X_{\gamma}\right)} & \leq \operatorname{const}_{M}\left\|d_{A_{\gamma}^{\prime}} \chi_{\gamma}+\alpha_{\gamma}\right\|_{L^{q}\left(X_{\gamma}^{\prime \prime}, g_{\gamma}\right)}, \\
& \leq \operatorname{const}_{M}^{\prime}\left\|d_{\boldsymbol{A}^{\prime}} \chi+j_{1}(\boldsymbol{v})+j_{2}(\boldsymbol{\alpha})\right\|_{B L^{q}}+\operatorname{const}_{M}\left\|\alpha_{\gamma}-j_{2}(\boldsymbol{\alpha})\right\|_{L^{q}\left(X_{\gamma}^{\prime \prime}, g_{\gamma}\right)} .
\end{aligned}
$$

By using the argument in the proof of Lemma 6.1, we get

$$
\left\|\alpha_{\gamma}-j_{2}(\boldsymbol{\alpha})\right\|_{L^{q}\left(X_{\gamma}^{\prime \prime}, g_{\gamma}\right)} \leq \operatorname{const}_{M} \cdot b^{4 / q}\|\boldsymbol{\alpha}\| .
$$

Since $b \ll 1$, we get the above conclusion.

Let $\chi \in \Omega^{0}(\operatorname{ad} \boldsymbol{E})_{0}$ and $\xi \in \Omega^{+}(\operatorname{ad} \boldsymbol{E})$ be smooth (not necessarily compact supported) 0 -form and self-dual form valued in $\operatorname{ad} \boldsymbol{E}$ over $X^{\sharp(\Gamma, S)}$, and let $\boldsymbol{v} \in \boldsymbol{V}$ and $\boldsymbol{\alpha} \in \boldsymbol{H}$. We define the norm $\|(\chi, \boldsymbol{v}, \boldsymbol{\alpha}, \xi)\|_{B_{1}}$ by

$$
\|(\chi, \boldsymbol{v}, \boldsymbol{\alpha}, \xi)\|_{B_{1}}:=\left\|d_{\boldsymbol{A}^{\prime}} \chi+j_{1}(\boldsymbol{v})+j_{2}(\boldsymbol{\alpha})\right\|_{B L^{q}}+\|\xi\|_{B L^{p}} .
$$

Lemma 6.2 shows that this becomes a norm. (Of course, its value might be infinity.) We define the Banach space $B_{1}$ as the completion of the space of $(\chi, \boldsymbol{v}, \boldsymbol{\alpha}, \xi) \in \Omega^{0}(\operatorname{ad} \boldsymbol{E})_{0} \oplus$ $\boldsymbol{V} \oplus \boldsymbol{H} \oplus \Omega^{+}(\operatorname{ad} \boldsymbol{E})$ of $\|(\chi, \boldsymbol{v}, \boldsymbol{\alpha}, \xi)\|_{B_{1}}<\infty$ in the norm $\|\cdot\|_{B_{1}}$ :

$$
B_{1}:=\overline{\left\{(\chi, \boldsymbol{v}, \boldsymbol{\alpha}, \xi) \in \Omega^{0}(\operatorname{ad} \boldsymbol{E})_{0} \oplus \boldsymbol{V} \oplus \boldsymbol{H} \oplus \Omega^{+}(\operatorname{ad} \boldsymbol{E}) \mid\|(\chi, \boldsymbol{v}, \boldsymbol{\alpha}, \xi)\|_{B_{1}}<\infty\right\}},
$$

where the overline means the completion in the norm $\|\cdot\|_{B_{1}}$. Let $\omega \in \Omega^{1}(\operatorname{ad} \boldsymbol{E})$ be a smooth 1 -form valued in ad $\boldsymbol{E}$ over $X^{\sharp(\Gamma, S)}$. We define the norm $\|\omega\|_{B_{2}}$ by setting

$$
\|\omega\|_{B_{2}}:=\|\omega\|_{B L^{q}}+\left\|d_{A^{\prime}}^{+} \omega\right\|_{B L^{p}} .
$$

We define the Banach space $B_{2}$ as the completion of the space of $\omega \in \Omega^{1}(\operatorname{ad} \boldsymbol{E})$ of $\|\omega\|_{B_{2}}<$ $\infty$ in the norm $\|\cdot\|_{B_{2}}$ :

$$
B_{2}:=\overline{\left\{\omega \in \Omega^{1}(\operatorname{ad} \boldsymbol{E}) \mid\|\omega\|_{B_{2}}<\infty\right\}} .
$$

Let $P=P_{\theta}: B L^{p} \rightarrow B L^{q}$ be the map defined in Lemma 4.1. $P$ is a right inverse of $d_{\boldsymbol{A}^{\prime}}^{+}$. We define a linear map $T: B_{1} \rightarrow B_{2}$ by

$$
T(\chi, \boldsymbol{v}, \boldsymbol{\alpha}, \xi):=d_{\boldsymbol{A}^{\prime}} \chi+j_{1}(\boldsymbol{v})+j_{2}(\boldsymbol{\alpha})+P(\xi) .
$$

Proposition 6.3. $T$ is a bounded linear operator. Moreover there exists a positive constant $K$ depending only on $M$ such that for any $(\chi, \boldsymbol{v}, \boldsymbol{\alpha}, \xi) \in B_{1}$

$$
\|(\chi, \boldsymbol{v}, \boldsymbol{\alpha}, \xi)\|_{B_{1}} \leq K\|T(\chi, \boldsymbol{v}, \boldsymbol{\alpha}, \xi)\|_{B_{2}} .
$$

Proof. Set $\omega:=T(\chi, \boldsymbol{v}, \boldsymbol{\alpha}, \xi)$. We have (using $d_{\boldsymbol{A}^{\prime}}^{+} j_{1}(v)=0$ )

$$
d_{\boldsymbol{A}^{\prime}}^{+} \omega=\left[F_{\boldsymbol{A}^{\prime}}^{+}, \chi\right]+d_{\boldsymbol{A}^{\prime}}^{+} j_{2}(\boldsymbol{\alpha})+\xi .
$$


Form Lemma 6.1 and $6.2, T$ is bounded. From this equation (remember $\left\|F^{+}\left(\boldsymbol{A}^{\prime}\right)\right\|_{B L^{p}} \leq$ const $\left._{M} b^{4 / p}\right)$

$$
\begin{aligned}
\|\xi\|_{B L^{p}} & \leq\|\omega\|_{B_{2}}+\operatorname{const}_{M} \cdot b^{4 / p}\left(\|\chi\|_{\mathcal{C}^{0}}+\|\boldsymbol{\alpha}\|\right) \\
& \leq\|\omega\|_{B_{2}}+\operatorname{const}_{M} \cdot b^{4 / p}\left\|d_{\boldsymbol{A}^{\prime}} \chi+j_{1}(\boldsymbol{v})+j_{2}(\boldsymbol{\alpha})\right\|_{B L^{q}} \\
& =\|\omega\|_{B_{2}}+\operatorname{const}_{M} \cdot b^{4 / p}\|\omega-P(\xi)\|_{B L^{q}} \\
& \leq\left(1+\operatorname{const}_{M} \cdot b^{4 / p}\right)\|\omega\|_{B_{2}}+\operatorname{const}_{M} \cdot b^{4 / p}\|\xi\|_{B L^{p}} .
\end{aligned}
$$

Since $b \ll 1$, we get $\|\xi\|_{B L^{p}} \leq 2\|\omega\|_{B_{2}}$ and

$$
\left\|d_{\boldsymbol{A}^{\prime}} \chi+j_{1}(\boldsymbol{v})+j_{2}(\boldsymbol{\alpha})\right\|_{B L^{p}}=\|\omega-P(\xi)\|_{B L^{p}} \leq \operatorname{const}_{M}\|\omega\|_{B_{2}} .
$$

This result shows that $T$ is an embedding. Indeed we want to prove that $T$ is an isomorphism. Donaldson-Kronheimer [5, Section 7.2.5] proves a similar result by using the index theorem. But we cannot use the index theorem and must prove it by a direct analysis.

Let $\omega \in B_{2}$ and set $\omega^{\prime}:=\omega-P d_{\boldsymbol{A}^{\prime}}^{+} \omega$. We have $d_{\boldsymbol{A}^{\prime}}^{+} \omega^{\prime}=0$ and $\left\|\omega^{\prime}\right\|_{B L^{q}} \leq \operatorname{const}_{M}\|\omega\|_{B_{2}}$. Consider $\beta_{\gamma} \omega^{\prime}$ on $X_{\gamma}$ for each $\gamma \in \Gamma$. We have $d_{A_{\gamma}}^{+}\left\{\beta_{\gamma} \omega^{\prime}-P_{\gamma} d_{A_{\gamma}}^{+}\left(\beta_{\gamma} \omega^{\prime}\right)\right\}=0$ where $P_{\gamma}=$ $\left(d_{A_{\gamma}}^{+}\right)^{*}\left(d_{A_{\gamma}}^{+}\left(d_{A_{\gamma}}^{+}\right)^{*}\right)^{-1}$ is the right inverse of $d_{A_{\gamma}}^{+}$. Then there uniquely exist $\chi_{\gamma} \in \Omega^{0}\left(\operatorname{ad} E_{\gamma}\right)$ and $\alpha_{\gamma} \in H_{A_{\gamma}}^{1}$ such that

$$
d_{A_{\gamma}} \chi_{\gamma}+\alpha_{\gamma}=\beta_{\gamma} \omega^{\prime}-P_{\gamma} d_{A_{\gamma}}^{+}\left(\beta_{\gamma} \omega^{\prime}\right),
$$

and $\chi_{\gamma}\left(z_{\gamma}\right)=0$ if $\gamma \in I$. (If $\gamma \in I$, then $H_{A_{\gamma}}^{1}=0$ and $\alpha_{\gamma}=0$.) Since $d_{\boldsymbol{A}^{\prime}}^{+} \omega^{\prime}=0$,

$$
P_{\gamma} d_{A_{\gamma}}^{+}\left(\beta_{\gamma} \omega^{\prime}\right)=P_{\gamma}\left(d \beta_{\gamma} \wedge \omega^{\prime}\right)^{+}+P_{\gamma}\left[\left(A_{\gamma}-A_{\gamma}^{\prime}\right) \wedge\left(\beta_{\gamma} \omega^{\prime}\right)\right]^{+}
$$

A difficulty comes from the term $P_{\gamma}\left(d \beta_{\gamma} \wedge \omega^{\prime}\right)^{+}$. The term $P_{\gamma}\left[\left(A_{\gamma}-A_{\gamma}^{\prime}\right) \wedge\left(\beta_{\gamma} \omega\right)\right]^{+}$can be easily estimated:

$$
\begin{aligned}
\left\|P_{\gamma}\left[\left(A_{\gamma}-A_{\gamma}^{\prime}\right) \wedge\left(\beta_{\gamma} \omega^{\prime}\right)\right]^{+}\right\|_{L^{q}\left(X_{\gamma}, g_{\gamma}\right)} & \leq \operatorname{const}_{M}\left\|A_{\gamma}-A_{\gamma}^{\prime}\right\|_{L^{4}}\left\|\beta_{\gamma} \omega^{\prime}\right\|_{L^{q}\left(X_{\gamma}^{\prime}, g_{\gamma}\right)} \\
& \leq \operatorname{const}_{M} \cdot b^{2} N^{2-8 / q}\|\omega\|_{B_{2}} .
\end{aligned}
$$

Here we have used (10) and (15). If we choose $b^{2} N^{2-8 / q} \ll 1$, then this is a good estimate. But a similar estimation gives

$$
\left\|P_{\gamma}\left(d \beta_{\gamma} \wedge \omega^{\prime}\right)^{+}\right\|_{L^{q}\left(X_{\gamma}, g_{\gamma}\right)} \leq \operatorname{const}_{M} \cdot N^{2-8 / q}\|\omega\|_{B_{2}} .
$$

Since $N \gg 1$ and $2-8 / q>0$, this is not a small term. We will come back to this point later. We have $\left(x_{\gamma}\left(z_{\gamma}\right)=0\right.$ for $\left.\gamma \in \Gamma\right)$

$$
\left\|\chi_{\gamma}\right\|_{\mathcal{C}^{0}}+\left\|\alpha_{\gamma}\right\|_{L^{q}\left(X_{\gamma}, g_{\gamma}\right)} \leq \operatorname{const}_{M}\left\|d_{A_{\gamma}} \chi_{\gamma}+\alpha_{\gamma}\right\|_{L^{q}\left(X_{\gamma}, g_{\gamma}\right)} \leq \operatorname{const}_{M} \cdot N^{2-8 / q}\|\omega\|_{B_{2}} .
$$


Set $\chi:=\sum_{\gamma} \beta_{\gamma}^{\prime} \chi_{\gamma} \in \Omega^{0}(\operatorname{ad} \boldsymbol{E})_{0}$. Then we have the following equation (using $\beta_{\gamma}^{\prime} \beta_{\gamma}=\beta_{\gamma}^{\prime}$ ):

$$
\begin{aligned}
& d_{\boldsymbol{A}^{\prime}} \chi-\sum_{\gamma} d \beta_{\gamma}^{\prime} \otimes \chi_{\gamma}+\sum_{\gamma} \beta_{\gamma}^{\prime} \alpha_{\gamma} \\
& =\omega^{\prime}+\sum_{\gamma}\left(\beta_{\gamma}^{\prime}\left[A_{\gamma}-A_{\gamma}^{\prime}, \chi_{\gamma}\right]-\beta_{\gamma}^{\prime} P_{\gamma}\left[\left(A_{\gamma}-A_{\gamma}^{\prime}\right) \wedge\left(\beta_{\gamma} \omega^{\prime}\right)\right]^{+}\right)-\sum_{\gamma} \beta_{\gamma}^{\prime} P_{\gamma}\left(d \beta_{\gamma} \wedge \omega^{\prime}\right)^{+} .
\end{aligned}
$$

From (51),

(53) $\left\|\left[A_{\gamma}-A_{\gamma}^{\prime}, \chi_{\gamma}\right]\right\|_{L^{q}\left(X_{\gamma}^{\prime \prime}, g\right)} \leq\left\|A_{\gamma}-A_{\gamma}^{\prime}\right\|_{L^{q}\left(X_{\gamma}^{\prime \prime}, g_{\gamma}\right)}\left\|\chi_{\gamma}\right\|_{\mathcal{C}^{0}} \leq \operatorname{const}_{M} \cdot b^{1+4 / q} N^{2-8 / q}\|\omega\|_{B_{2}}$.

$$
\begin{aligned}
\left\|P_{\gamma}\left[\left(A_{\gamma}-A_{\gamma}^{\prime}\right) \wedge\left(\beta_{\gamma} \omega^{\prime}\right)\right]^{+}\right\|_{L^{q}\left(X_{\gamma}^{\prime \prime}, g\right)} & \leq \operatorname{const}_{M}\left\|A_{\gamma}-A_{\gamma}^{\prime}\right\|_{L^{4}}\left\|\beta_{\gamma} \omega\right\|_{L^{q}\left(X_{\gamma}^{\prime}, g_{\gamma}\right)} \\
& \leq \operatorname{const}_{M} \cdot b^{2} N^{2-8 / q}\|\omega\|_{B_{2}} .
\end{aligned}
$$

Hence

$$
\begin{aligned}
\left\|\sum_{\gamma}\left(\beta_{\gamma}^{\prime}\left[A_{\gamma}-A_{\gamma}^{\prime}, \chi_{\gamma}\right]-\beta_{\gamma}^{\prime} P_{\gamma}\left[\left(A_{\gamma}-A_{\gamma}^{\prime}\right) \wedge\left(\beta_{\gamma} \omega^{\prime}\right)\right]^{+}\right)\right\|_{B L^{q}} & \leq \operatorname{const}_{M} \cdot b^{1+4 / q} N^{2-8 / q}\|\omega\|_{B_{2}}, \\
& \leq \operatorname{const}_{M} \cdot b N^{2}\|\omega\|_{B_{2}} .
\end{aligned}
$$

The estimation of the term $\sum_{\gamma} \beta_{\gamma}^{\prime} P_{\gamma}\left(d \beta_{\gamma} \wedge \omega^{\prime}\right)^{+}$needs the following lemma:

Lemma 6.4. Let $0<\delta<1$, and $f$ be a $L^{p}$-function in $\mathbb{R}^{4}$ satisfying

$$
\operatorname{supp} f \subset\left\{x|\sqrt{\lambda} / N \leq| x \mid \leq \sqrt{\lambda} / N^{1-\delta}\right\}
$$

where $N \gg 1$. Set

$$
F(x):=\int_{\mathbb{R}^{4}} \frac{f(y)}{|x-y|^{3}} d y
$$

Then we have

$$
\left(\int_{|x| \geq \sqrt{\lambda} / 2}|F(x)|^{q} d x\right)^{1 / q} \leq \mathrm{const} \cdot N^{-4(1-1 / p)(1-\delta)}\|f\|_{L^{p}\left(\mathbb{R}^{4}\right)} .
$$

Here remember that $2<p<4, q>4$ and $1-4 / p=-4 / q$.

Proof. Using a scale change, we suppose $\lambda=1$ without loss of generality. If $|x| \geq 1 / 2$ and $|y| \leq N^{-1+\delta}$, then (using $N \gg 1$ and $-1+\delta<0$ ) $|x-y| \geq|x|-N^{-1+\delta} \geq|x| / 2$. Then $1 /|x-y|^{3} \leq 2^{3} /|x|^{3}$. Hence for $|x| \geq 1 / 2$

$$
|F(x)| \leq \frac{2^{3}}{|x|^{3}} \int_{\mathbb{R}^{4}}|f(y)| d y .
$$

Using $q>4$, we get

$$
\int_{|x| \geq 1 / 2}|F(x)|^{q} d x \leq 2^{3 q} \int_{1 / 2}^{\infty} \frac{d r}{r^{3 q-3}}\left(\int_{\mathbb{R}^{4}}|f(y)| d y\right)^{q} \leq \operatorname{const}\left(\int_{\mathbb{R}^{4}}|f(y)| d y\right)^{q} .
$$




$$
\begin{aligned}
\left(\int_{|x| \geq 1 / 2}|F(x)|^{q} d x\right)^{1 / q} & \leq \mathrm{const} \int_{\mathbb{R}^{4}}|f(y)| d y \\
& \leq \mathrm{const}\|f\|_{L^{p}\left(\mathbb{R}^{4}\right)}(\operatorname{vol}(\operatorname{supp} f))^{1-1 / p} \\
& \leq \mathrm{const} \cdot N^{-4(1-\delta)(1-1 / p)}\|f\|_{L^{p}\left(\mathbb{R}^{4}\right)}
\end{aligned}
$$

We use this lemma for $\delta=1 / 6$.

\section{Lemma 6.5.}

$$
\left\|P_{\gamma}\left(d \beta_{\gamma} \wedge \omega^{\prime}\right)^{+}\right\|_{L^{q}\left(X_{\gamma}^{\prime \prime}, g_{\gamma}\right)} \leq \operatorname{const}_{M} \cdot N^{-1 / 2}\|\omega\|_{B_{2}}
$$

Proof. Set $\sigma:=\left(d \beta_{\gamma} \wedge \omega^{\prime}\right)^{+}$and $\delta=1 / 6$. There exists $r_{0}>0$ (independent of $\lambda$ and $N$ ) such that the metric $g_{\gamma}$ is flat over the balls $B_{s}=B\left(x_{\gamma, s}, r_{0}\right)$ and $B_{s}^{\prime}=B\left(y_{\gamma, s}, r_{0}\right)(s \in S)$. We assume $r_{0} \gg b=4 N \sqrt{\lambda}$. Set $B:=\bigcup\left(B_{s} \cup B_{s}^{\prime}\right)$. We define the annulus region $A_{s}$ and $A_{s}^{\prime}$ by $A_{s}:=B\left(x_{\gamma, s}, \sqrt{\lambda} / N^{1-\delta}\right) \backslash \bar{B}\left(x_{\gamma, s}, \sqrt{\lambda} / N\right)$ and $A_{s}^{\prime}:=B\left(y_{\gamma, s}, \sqrt{\lambda} / N^{1-\delta}\right) \backslash$ $\bar{B}\left(y_{\gamma, s}, \sqrt{\lambda} / N\right)$, and set $A:=\bigcup\left(A_{s} \cup A_{s}^{\prime}\right)$. Remember that $\operatorname{supp} \sigma \subset \operatorname{supp}\left(d \beta_{\gamma}\right) \subset A$ by Lemma 3.4.

$P_{\gamma} \sigma$ can be expressed by using the Green kernel:

$$
P_{\gamma} \sigma(x)=\int_{A} G(x, y) \sigma(y) d \operatorname{vol}(y),
$$

where the volume form $d \operatorname{vol}(y)=d \operatorname{vol}_{g_{\gamma}}(y)$ is defined by using the metric $g_{\gamma}$. The Green kernel $G(x, y)$ has a singularity of degree 3 along the diagonal (cf. Donaldson [4, p. 310]):

$$
|G(x, y)| \leq \text { const }_{M} / d(x, y)^{3},
$$

where $d(x, y)$ is the distance on $X_{\gamma}$ defined by $g_{\gamma}$.

$$
\int_{X_{\gamma}^{\prime \prime}}\left|P_{\gamma} \sigma\right|^{q} d \mathrm{vol}=\int_{X_{\gamma}^{\prime \prime} \backslash B}\left|P_{\gamma} \sigma\right|^{q} d \mathrm{vol}+\int_{B \cap X_{\gamma}^{\prime \prime}}\left|P_{\gamma} \sigma\right|^{q} d \mathrm{vol}
$$

The first term can be easily estimated:

$$
\begin{aligned}
\int_{X_{\gamma}^{\prime \prime} \backslash B}\left|P_{\gamma} \sigma\right|^{q} d \mathrm{vol} & \leq \operatorname{const}_{M} \int_{X_{\gamma}^{\prime \prime}} d \operatorname{vol}(x)\left(\int_{A}|\sigma(y)| d \operatorname{vol}(y)\right)^{q} \\
& \leq \operatorname{const}_{M} \cdot(\operatorname{vol} A)^{q(1-1 / p)}\left(\int_{A}|\sigma(y)|^{p} d \operatorname{vol}(y)\right)^{q / p} \\
& \leq \text { const }_{M} \cdot\left(\lambda^{2} N^{-4(1-\delta)}\right)^{q(1-1 / p)}\left(\int_{A}|\sigma(y)|^{p} d \operatorname{vol}(y)\right)^{q / p}
\end{aligned}
$$

From Lemma 6.4,

$$
\left(\int_{B \cap X_{\gamma}^{\prime \prime}}\left|P_{\gamma} \sigma\right|^{q} d \mathrm{vol}\right)^{1 / q} \leq \operatorname{const}_{M} \cdot N^{-4(1-\delta)(1-1 / p)}\left(\int_{A}|\sigma(y)|^{p} d \operatorname{vol}(y)\right)^{1 / p} .
$$


Hence

From (10),

$\|\sigma\|_{L^{p}\left(X_{\gamma}^{\prime}, g_{\gamma}\right)} \leq\left\|d \beta_{\gamma}\right\|_{L^{4}}\left\|\omega^{\prime}\right\|_{L^{q}\left(X_{\gamma}^{\prime}, g_{\gamma}\right)} \leq \operatorname{const}_{M} \cdot N^{2-8 / q}\left\|\omega^{\prime}\right\|_{L^{q}\left(X_{\gamma}^{\prime}, g\right)} \leq \operatorname{const}_{M} \cdot N^{2-8 / q}\|\omega\|_{B_{2}}$. We have $1-4 / p=-4 / q, 2<p<4$ and $\delta=1 / 6$. Then $2-8 / q-4(1-\delta)(1-1 / p)=$ $4 \delta(1-1 / p)-4 / p<-1 / 2$. Therefore we get the above conclusion.

From the above Lemma, we get

$$
\left\|\sum_{\gamma} \beta_{\gamma}^{\prime} P_{\gamma}\left(d \beta_{\gamma} \wedge \omega^{\prime}\right)^{+}\right\|_{B L^{q}} \leq \operatorname{const}_{M} \cdot N^{-1 / 2}\|\omega\|_{B_{2}} .
$$

From the equation (52) and the estimate (55), we get

$$
\left\|d_{\boldsymbol{A}^{\prime}} \chi-\sum_{\gamma} d \beta_{\gamma}^{\prime} \otimes \chi_{\gamma}+\sum_{\gamma} \beta_{\gamma}^{\prime} \alpha_{\gamma}-\omega^{\prime}\right\|_{B L^{q}} \leq \operatorname{const}_{M}\left(b N^{2}+N^{-1 / 2}\right)\|\omega\|_{B_{2}} .
$$

Let $W_{\gamma, s}:=B\left(x_{\gamma, s}, 2 \sqrt{\lambda}\right) \backslash B\left(x_{\gamma, s}, \sqrt{\lambda} / 2\right) \subset X_{\gamma}$ be the "neck" region $(\gamma \in \Gamma, s \in S)$. Since $d \beta_{\gamma}^{\prime}=-d \beta_{\gamma s}^{\prime}$ over $W_{\gamma, s}$, the term $-\sum_{\gamma} d \beta_{\gamma}^{\prime} \otimes \chi_{\gamma}$ can be expressed by

$$
-\sum_{\gamma} d \beta_{\gamma}^{\prime} \otimes \chi_{\gamma}=\left.\sum_{\gamma, s} d \beta_{\gamma}^{\prime} \otimes\left(-\chi_{\gamma}+\chi_{\gamma s}\right)\right|_{W_{\gamma, s}} .
$$

We have $d_{A_{\gamma}} \chi_{\gamma}=\beta_{\gamma} \omega^{\prime}-P_{\gamma} d_{A_{\gamma}}^{+}\left(\beta_{\gamma} \omega^{\prime}\right)-\alpha_{\gamma}$. Since $\beta_{\gamma}=1$ over the neck $W_{\gamma, s}$, we have

$$
d_{\boldsymbol{A}^{\prime}} \chi_{\gamma}=\omega^{\prime}-P_{\gamma} d_{A_{\gamma}}^{+}\left(\beta_{\gamma} \omega^{\prime}\right)-\alpha_{\gamma}+\left[A_{\gamma}^{\prime}-A_{\gamma}, \chi_{\gamma}\right] \text { on } W_{\gamma, s} \text {. }
$$

Therefore on the neck $W_{\gamma, s}$

$$
\begin{aligned}
& d_{\boldsymbol{A}^{\prime}}\left(\chi_{\gamma}-\chi_{\gamma s}\right) \\
& =-P_{\gamma} d_{A_{\gamma}}^{+}\left(\beta_{\gamma} \omega^{\prime}\right)-\alpha_{\gamma}+\left[A_{\gamma}^{\prime}-A_{\gamma}, \chi_{\gamma}\right]+P_{\gamma s} d_{A_{\gamma s}}^{+}\left(\beta_{\gamma s} \omega^{\prime}\right)+\alpha_{\gamma s}-\left[A_{\gamma s}^{\prime}-A_{\gamma s}, \chi_{\gamma s}\right] .
\end{aligned}
$$

As in (54) and Lemma 6.5,

$$
\left\|P_{\gamma} d_{A_{\gamma}}^{+}\left(\beta_{\gamma} \omega^{\prime}\right)\right\|_{L^{q}\left(W_{\gamma, s}, g\right)} \leq \operatorname{const}_{M} \cdot\left(N^{-1 / 2}+b^{2} N^{2-8 / q}\right)\|\omega\|_{B_{2}} .
$$

From (53),

$$
\left\|\left[A_{\gamma}-A_{\gamma}^{\prime}, \chi_{\gamma}\right]\right\|_{L^{q}\left(X_{\gamma}^{\prime \prime}, g\right)} \leq \operatorname{const}_{M} \cdot b^{1+4 / q} N^{2-8 / q}\|\omega\|_{B_{2}} .
$$

From (51) and an (elliptic) estimate $\left\|\alpha_{\gamma}\right\|_{L^{\infty}\left(X_{\gamma}, g_{\gamma}\right)} \leq \operatorname{const}_{M}\left\|\alpha_{\gamma}\right\|_{L^{q}\left(X_{\gamma}, g_{\gamma}\right)}$,

$$
\begin{aligned}
\left\|\alpha_{\gamma}\right\|_{L^{q}\left(W_{\gamma, s}, g\right)} & \leq\left(\text { const } \cdot \lambda^{2}\right)^{1 / q}\left\|\alpha_{\gamma}\right\|_{L^{\infty}\left(X_{\gamma}, g_{\gamma}\right)}, \\
& \leq \operatorname{const}_{M} \cdot \lambda^{2 / q}\left\|\alpha_{\gamma}\right\|_{L^{q}\left(X_{\gamma}, g_{\gamma}\right)} \leq \operatorname{const}_{M} \cdot \lambda^{2 / q} N^{2-8 / q}\|\omega\|_{B_{2}} .
\end{aligned}
$$

In the same way, we get the estimates of the other terms in the right-hand-side of $(57)$. Then

$$
\left\|d_{\boldsymbol{A}^{\prime}}\left(\chi_{\gamma}-\chi_{\gamma s}\right)\right\|_{L^{q}\left(W_{\gamma, s}, g\right)} \leq \operatorname{const}_{M} \cdot\left(N^{-1 / 2}+b N^{2}+\lambda^{2 / q} N^{2}\right)\|\omega\|_{B_{2}} .
$$


Let $v_{\gamma, s} \in\left(\operatorname{ad} E_{\gamma}\right)_{x_{\gamma, s}} \cong\left(\operatorname{ad} E_{\gamma s}\right)_{y_{\gamma s, s}}$ be the mean value of $\chi_{\gamma s}-\chi_{\gamma}$ over the neck $W_{\gamma, s}$. Using the Sobolev embedding $L_{1}^{q} \hookrightarrow \mathcal{C}^{0,1-4 / q}$ (Hölder space), we get

$$
\begin{aligned}
\left\|\chi_{\gamma s}-\chi_{\gamma}-v_{\gamma, s}\right\|_{\mathcal{C}^{0}\left(W_{\gamma, s}\right)} & \leq \text { const } \cdot \lambda^{1 / 2-2 / q}\left\|d_{A^{\prime}}\left(\chi_{\gamma}-\chi_{\gamma s}\right)\right\|_{L^{q}\left(W_{\gamma, s}, g\right)}, \\
& \leq \operatorname{const}_{M} \cdot \lambda^{1 / 2-2 / q}\left(N^{-1 / 2}+b N^{2}+\lambda^{2 / q} N^{2}\right)\|\omega\|_{B_{2}} .
\end{aligned}
$$

Set $\boldsymbol{v}:=\left(v_{\gamma, s}\right)_{\gamma, s} \in \boldsymbol{V}$.

$$
\begin{aligned}
\left\|\sum_{\gamma} d \beta_{\gamma}^{\prime} \otimes \chi_{\gamma}+j_{1}(\boldsymbol{v})\right\|_{B L^{q}} & =\left\|\left.\sum_{\gamma, s} d \beta_{\gamma}^{\prime} \otimes\left(v_{\gamma, s}-\chi_{\gamma s}+\chi_{\gamma}\right)\right|_{W_{\gamma, s}}\right\|_{B L^{q}}, \\
& \leq \operatorname{const}_{M} \cdot \frac{1}{\sqrt{\lambda}}(\sqrt{\lambda})^{4 / q} \lambda^{1 / 2-2 / q}\left(N^{-1}+b N^{2}+\lambda^{2 / q} N^{2}\right)\|\omega\|_{B_{2}}, \\
& \leq \operatorname{const}_{M}\left(N^{-1 / 2}+b N^{2}+\lambda^{2 / q} N^{2}\right)\|\omega\|_{B_{2}} .
\end{aligned}
$$

Set $\boldsymbol{\alpha}:=\left(\alpha_{\gamma}\right)_{\gamma} \in \boldsymbol{H}$. From (51), $\|\boldsymbol{\alpha}\|=\sup \left\|\alpha_{\gamma}\right\|_{L^{q}} \leq \operatorname{const}_{M} \cdot N^{2-8 / q}\|\omega\|_{B_{2}}$. Using Lemma 6.1 , we get $(b=4 N \sqrt{\lambda})$

$$
\begin{aligned}
\left\|j_{2}(\boldsymbol{\alpha})-\sum_{\gamma \in \Gamma} \beta_{\gamma}^{\prime} \alpha_{\gamma}\right\|_{B L^{q}} & \leq \operatorname{const}_{M} \cdot b^{4 / q}\|\boldsymbol{\alpha}\| \leq \operatorname{const}_{M} \cdot b^{4 / q} N^{2-8 / q}\|\omega\|_{B_{2}}, \\
& \leq \text { const }_{M} \cdot \lambda^{2 / q} N^{2}\|\omega\|_{B_{2}} .
\end{aligned}
$$

Using this and (58) in the estimate (56), we get

$$
\left\|d_{\boldsymbol{A}^{\prime}} \chi+j_{1}(\boldsymbol{v})+j_{2}(\boldsymbol{\alpha})-\omega^{\prime}\right\|_{B L^{q}} \leq \operatorname{const}_{M} \cdot\left(N^{-1 / 2}+b N^{2}+\lambda^{2 / q} N^{2}\right)\|\omega\|_{B_{2}} .
$$

We have $d_{\boldsymbol{A}^{\prime}}^{+}\left(d_{\boldsymbol{A}^{\prime}} \chi+j_{1}(\boldsymbol{v})+j_{2}(\boldsymbol{\alpha})-\omega^{\prime}\right)=\left[F_{\boldsymbol{A}^{\prime}}^{+}, \chi\right]+d_{\boldsymbol{A}^{\prime}}^{+} j_{2}(\boldsymbol{\alpha})$. Using $\left\|F_{\boldsymbol{A}^{\prime}}^{+}\right\|_{B L^{p}} \leq$ const $_{M^{\prime}}$. $b^{4 / p},(51)$ and Lemma 6.1, we get

$$
\begin{aligned}
\left\|\left[F_{\boldsymbol{A}^{\prime}}^{+}, \chi\right]+d_{\boldsymbol{A}^{\prime}}^{+} j_{2}(\boldsymbol{\alpha})\right\|_{B L^{p}} & \leq \text { const }_{M} \cdot b^{4 / p} N^{2-8 / q}\|\omega\|_{B_{2}}+\text { const }_{M} \cdot b^{4 / p}\|\boldsymbol{\alpha}\|, \\
& \leq \operatorname{const}_{M} \cdot \lambda^{2 / q} N^{2}\|\omega\|_{B_{2}} .
\end{aligned}
$$

Thus we conclude that

$$
\left\|d_{\boldsymbol{A}^{\prime}} \chi+j_{1}(\boldsymbol{v})+j_{2}(\boldsymbol{\alpha})-\omega^{\prime}\right\|_{B_{2}} \leq \operatorname{const}_{M} \cdot\left(N^{-1 / 2}+b N^{2}+\lambda^{2 / q} N^{2}\right)\|\omega\|_{B_{2}} .
$$

We define a bounded linear operator $T^{\prime}: B_{2} \rightarrow B_{1}$ by $T^{\prime}(\omega):=\left(\chi, \boldsymbol{v}, \boldsymbol{\alpha}, d_{\boldsymbol{A}^{\prime}}^{+} \omega\right)$. Remember $\omega^{\prime}=\omega-P d_{\boldsymbol{A}^{\prime}}^{+} \omega$. The above shows $\left\|T T^{\prime}(\omega)-\omega\right\|_{B_{2}} \leq \operatorname{const}_{M} \cdot\left(N^{-1 / 2}+b N^{2}+\right.$ $\left.\lambda^{2 / q} N^{2}\right)\|\omega\|_{B_{2}}$. Therefore if we choose $\lambda$ and $N$ appropriately, then $\left(T T^{\prime}\right)^{-1}$ exists and $T^{\prime}\left(T T^{\prime}\right)^{-1}$ becomes a right inverse of $T$. In particular, $T$ becomes surjective and hence isomorphic (see Proposition 6.3). Then we get the following.

Proposition 6.6. There are $N_{0}>0$ and $\lambda_{0}(N)>0$ such that if $N \geq N_{0}$ and $\lambda \leq \lambda_{0}(N)$ then $T: B_{1} \rightarrow B_{2}$ (given in (48)) is an isomorphism and satisfies

$$
\|(\chi, \boldsymbol{v}, \boldsymbol{\alpha}, \xi)\|_{B_{1}} \leq K\|T(\chi, \boldsymbol{v}, \boldsymbol{\alpha}, \xi)\|_{B_{2}},
$$

where $K$ is a positive constant depending only on $M$. 
6.2. Some continuities. Let's recall our situation. $\Gamma$ is a finitely generated group and $S$ is its finite generating set which does not contain the identity element $e$. The group $\Gamma$ can be considered as a metric space endowed with the (left-invariant) word distance by $S:$ For $\gamma, \gamma^{\prime} \in \Gamma$,

$$
d_{S}\left(\gamma, \gamma^{\prime}\right):=\min \left\{n \geq 0 \mid \exists \gamma_{1}, \cdots, \gamma_{n} \in S \cup S^{-1}: \gamma^{-1} \gamma^{\prime}=\gamma_{1} \cdots \gamma_{n}\right\}
$$

For a subset $\Omega \subset \Gamma$ and an integer $d>0$, we set

$$
B_{d}(\Omega):=\left\{\gamma \in \Gamma \mid \exists \gamma^{\prime} \in \Omega: d_{S}\left(\gamma, \gamma^{\prime}\right) \leq d\right\}
$$

We define a open set $X_{\Omega} \subset X$ by

$$
X_{\Omega}:=\bigcup_{\gamma \in \Omega} X_{\gamma}^{\prime \prime} .
$$

Let $\theta_{i}=\left(E_{i \gamma}, A_{i \gamma}, \rho_{i \gamma, s}\right)_{\gamma \in \Gamma, s \in S}(i=1,2)$ be two gluing data, i.e., $E_{i \gamma}$ is a principal $S U(2)$-bundle over $X_{\gamma}$ and $A_{i \gamma}$ is an ASD connection on $E_{i \gamma}$ satisfying $\left[E_{i \gamma}, A_{i \gamma}\right] \in M$. $\rho_{i \gamma, s}:\left(E_{i \gamma}\right)_{x_{\gamma, s}} \rightarrow\left(E_{i \gamma s}\right)_{y_{\gamma s, s}}$ is an $S U(2)$-isomorphism. For each $i=1,2$, we have the operator $P_{i}: B L^{p}\left(\Omega^{+}\left(\operatorname{ad} \boldsymbol{E}_{i}\right)\right) \rightarrow B L^{q}\left(\Omega^{1}\left(\operatorname{ad} \boldsymbol{E}_{i}\right)\right)$ which is a right inverse of $d_{\boldsymbol{A}_{i}^{\prime}}^{+}$by Lemma 4.1. Let $\Omega \subset \Gamma$ be a finite set. We want to compare the operators $P_{1}$ and $P_{2}$ over $X_{\Omega}$. Suppose that there is an integer $d>0$ such that $E_{1 \gamma}=E_{2 \gamma}, A_{1 \gamma}=A_{2 \gamma}$ for $\gamma \in B_{d}(\Omega)$ and $\rho_{1 \gamma, s}=\rho_{2 \gamma, s}$ for $\gamma \in B_{d}(\Omega)$ and $s \in S$ with $\gamma s \in B_{d}(\Omega)$. Then we can naturally consider that $\boldsymbol{E}_{1}=\boldsymbol{E}_{2}$ and $\boldsymbol{A}_{1}^{\prime}=\boldsymbol{A}_{2}^{\prime}$ over $X_{B_{d}(\Omega)}$.

Lemma 6.7. Let $\xi_{i} \in B L_{i}^{p}(i=1,2)$. We denote $\left.\xi_{i}\right|_{X_{B_{d}(\Omega)}}$ as the restriction of $\xi_{i}$ to $X_{B_{d}(\Omega)}$ (and we extend it to $X^{\sharp(\Gamma, S)}$ by zero). Then for each $\gamma \in \Omega$

$\left\|P_{1}\left(\xi_{1}\right)-P_{2}\left(\xi_{2}\right)\right\|_{L^{q}\left(X_{\gamma}^{\prime \prime}, g\right)} \leq \operatorname{const}_{M}\left\|\left.\xi_{1}\right|_{B_{d}(\Omega)}-\left.\xi_{2}\right|_{B_{d}(\Omega)}\right\|_{B L^{p}}+\operatorname{const}_{M} \cdot 2^{-d}\left(\left\|\xi_{1}\right\|_{B L^{p}}+\left\|\xi_{2}\right\|_{B L^{p}}\right)$, where const $_{M}$ are positive constants depending only on $M$. (Especially they are independent of $\Omega$ and the integer $d>0$.) In particular, if $\left.\xi_{1}\right|_{X_{B_{d}(\Omega)}}=\left.\xi_{2}\right|_{X_{B_{d}(\Omega)}}$ then

$$
\left\|P_{1}\left(\xi_{1}\right)-P_{2}\left(\xi_{2}\right)\right\|_{L^{q}\left(X_{\gamma}^{\prime \prime}, g\right)} \leq \operatorname{const}_{M} \cdot 2^{-d}\left(\left\|\xi_{1}\right\|_{B L^{p}}+\left\|\xi_{2}\right\|_{B L^{p}}\right)
$$

Proof.

$$
\begin{aligned}
P_{i}\left(\xi_{i}\right) & =Q_{i}\left(1+R_{i}\right)^{-1} \xi_{i}, \\
& =Q_{i}\left(1-R_{i}+R_{i}^{2}-\cdots+(-1)^{d-1} R_{i}^{d-1}\right) \xi_{i}+(-1)^{d} Q_{i} R_{i}^{d}\left(1+R_{i}\right)^{-1} \xi_{i} .
\end{aligned}
$$

From the definitions of the operators $Q$ and $R$ in Section 4 , we have $Q_{i} R_{i}^{k} \xi_{i}=Q_{i} R_{i}^{k}\left(\left.\xi_{i}\right|_{X_{B_{d}(\Omega)}}\right)$ and $Q_{2} R_{2}^{k}\left(\left.\xi_{2}\right|_{X_{B_{d}(\Omega)}}\right)=Q_{1} R_{1}^{k}\left(\left.\xi_{2}\right|_{X_{B_{d}(\Omega)}}\right)$ over $X_{\Omega}$ for $k \leq d-1$. (These follows from the fact that $Q_{i}$ and $R_{i}$ have "one-step propagation".) Therefore for $\gamma \in \Omega$

$\left\|P_{1}\left(\xi_{1}\right)-P_{2}\left(\xi_{2}\right)\right\|_{L^{q}\left(X_{\gamma}^{\prime \prime}, g\right)} \leq \operatorname{const}_{M}\left\|\left.\xi_{1}\right|_{B_{d}(\Omega)}-\left.\xi_{2}\right|_{B_{d}(\Omega)}\right\|_{B L^{p}}+\operatorname{const}_{M} \cdot 2^{-d}\left(\left\|\xi_{1}\right\|_{B L^{p}}+\left\|\xi_{2}\right\|_{B L^{p}}\right)$.

Here we have used $\left\|R_{i}\right\| \leq 1 / 2$ (see Lemma 4.1).

The following will be used in Section 7 . 
Corollary 6.8. For any $\varepsilon>0$, there exists $d=d(M, \varepsilon)>0$ satisfying the following: Let $\Omega \subset \Gamma$ be a finite subset. If $E_{1 \gamma}=E_{2 \gamma}, A_{1 \gamma}=A_{2 \gamma}$ for all $\gamma \in B_{d}(\Omega)$ and $\rho_{1 \gamma, s}=\rho_{2 \gamma, s}$ for all $\gamma \in B_{d}(\Omega)$ and $s \in S$ with $\gamma s \in B_{d}(\Omega)$, then for any $\gamma \in \Omega$

$$
\left\|\boldsymbol{A}\left(\theta_{1}\right)-\boldsymbol{A}\left(\theta_{2}\right)\right\|_{L^{q}\left(X_{\gamma}^{\prime \prime}, g\right)}<\varepsilon .
$$

Proof. $\boldsymbol{\xi}_{i}=\boldsymbol{\xi}\left(\theta_{i}\right)$ satisfies $(i=1,2)$

$$
\boldsymbol{\xi}_{i}+\left(P_{i}\left(\boldsymbol{\xi}_{i}\right) \wedge P_{i}\left(\boldsymbol{\xi}_{i}\right)\right)^{+}=-F^{+}\left(\boldsymbol{A}_{i}^{\prime}\right) .
$$

Let $m$ and $d_{0}$ be (large) positive integers which will be fixed later. Set $d:=m d_{0}$ and suppose that $\theta_{1}=\theta_{2}$ over $B_{d}(\Omega)$. Since we have $\boldsymbol{A}_{1}^{\prime}=\boldsymbol{A}_{2}^{\prime}$ over $B_{d}(\Omega)$, we have

$$
\begin{aligned}
\boldsymbol{\xi}_{1}-\boldsymbol{\xi}_{2} & =\left(P_{2}\left(\boldsymbol{\xi}_{2}\right) \wedge P_{2}\left(\boldsymbol{\xi}_{2}\right)\right)^{+}-\left(P_{1}\left(\boldsymbol{\xi}_{1}\right) \wedge P_{1}\left(\boldsymbol{\xi}_{1}\right)\right)^{+} \\
& =\left(\left(P_{2}\left(\boldsymbol{\xi}_{2}\right)-P_{1}\left(\boldsymbol{\xi}_{1}\right)\right) \wedge P_{2}\left(\boldsymbol{\xi}_{2}\right)\right)^{+}+\left(P_{1}\left(\boldsymbol{\xi}_{1}\right) \wedge\left(\left(P_{2}\left(\boldsymbol{\xi}_{2}\right)-P_{1}\left(\boldsymbol{\xi}_{1}\right)\right)\right)^{+}\right.
\end{aligned}
$$

over $B_{d}(\Omega)$. For $k=1,2, \cdots, m$, we set

$$
a_{k}:=\sup _{\gamma \in B_{k d_{0}}(\Omega)}\left\|\boldsymbol{\xi}_{1}-\boldsymbol{\xi}_{2}\right\|_{L^{p}\left(X_{\gamma}^{\prime \prime}, g\right)} .
$$

From Remark 4.4, Lemma 6.7 and (59), we have

$$
a_{k} \leq \operatorname{const}_{M} \cdot b^{2} \sup _{\gamma \in B_{k d_{0}}(\Omega)}\left\|P_{2}\left(\boldsymbol{\xi}_{2}\right)-P_{1}\left(\boldsymbol{\xi}_{1}\right)\right\|_{L^{q}\left(X_{\gamma}^{\prime \prime}, g\right)} \leq \operatorname{const}_{M} \cdot b^{2}\left(a_{k+1}+b^{4 / p} 2^{-d_{0}}\right),
$$

where const $_{M}$ is independent of $k$ and $\Omega$. Since $b>0$ is sufficiently small, we have

$$
a_{k} \leq 2^{-1} a_{k+1}+2^{-d_{0}} \text {. }
$$

Hence $a_{1} \leq 2^{-m+1} a_{m}+2^{-d_{0}+1}$. We have $a_{m} \leq\left\|\boldsymbol{\xi}_{1}\right\|_{B L^{p}}+\left\|\boldsymbol{\xi}_{2}\right\|_{B L^{p}} \leq$ const $_{M} b^{4 / p} \ll 1$. Hence

$$
a_{1} \leq 2^{-m+1}+2^{-d_{0}+1} \text {. }
$$

We have $\boldsymbol{A}_{1}-\boldsymbol{A}_{2}=P_{1}\left(\boldsymbol{\xi}_{1}\right)-P_{2}\left(\boldsymbol{\xi}_{2}\right)$ over $B_{d}(\Omega)$, and for any $\gamma \in \Omega$ (using Lemma 6.7)

$$
\left\|P_{1}\left(\boldsymbol{\xi}_{\mathbf{1}}\right)-P_{2}\left(\boldsymbol{\xi}_{2}\right)\right\|_{L^{q}\left(X_{\gamma}^{\prime \prime}, g\right)} \leq \operatorname{const}_{M}\left(a_{1}+b^{4 / p} \cdot 2^{-d_{0}}\right) .
$$

We choose $m$ and $d_{0}$ sufficiently large. Then for $\gamma \in \Gamma$

$$
\left\|P_{1}\left(\boldsymbol{\xi}_{\mathbf{1}}\right)-P_{2}\left(\boldsymbol{\xi}_{2}\right)\right\|_{L^{q}\left(X_{\gamma}^{\prime \prime}, g\right)}<\varepsilon \text {. }
$$

Let $\left[\theta_{n}\right]=\left[\left(E_{n \gamma}, A_{n \gamma}, \rho_{n \gamma, s}\right)_{\gamma \in \Gamma, s \in S}\right] \in \operatorname{GID}(n=1,2,3, \cdots)$ be the sequence of the equivalence classes of gluing data. Since $M$ is compact, if we take a subsequence, this sequence (pointwisely) converges to a gluing data $[\theta]=\left[\left(E_{\gamma}, A_{\gamma}, \rho_{\gamma, s}\right)_{\gamma \in \Gamma, s \in S}\right]$ in the following sense (cf. Section 7). For each $\gamma \in \Gamma$ there exists $n_{0}(\gamma)>0$ and a sequence of gauge transformations $g_{n \gamma}: E_{n \gamma} \rightarrow E_{\gamma}\left(n \geq n_{0}(\gamma)\right)$ such that $g_{n \gamma}\left(A_{n \gamma}\right)$ converges to $A_{\gamma}$ (in the $\mathcal{C}^{\infty}$-topology) and $g_{n \gamma s} \rho_{n \gamma, s} g_{n \gamma}^{-1}$ converges to $\rho_{\gamma, s}$ as $n \rightarrow \infty$.

For each $\gamma \in \Gamma$ we can assume that, for $n \geq n_{0}(\gamma), E_{n \gamma}=E_{\gamma}, A_{n \gamma}=A_{\gamma}+\tilde{\alpha}_{n \gamma}$ and $\rho_{n \gamma, s}=\rho_{\gamma, s} e^{v_{n \gamma, s}}(s \in S)$ where $\alpha_{n \gamma} \in H_{A_{\gamma}}^{1}$ and $v_{n \gamma, s} \in(\operatorname{ad} E)_{x_{\gamma, s}}$. (See Section 3.4.2 and 
5.1). Moreover we have $\lim _{n \rightarrow \infty}\left\|\alpha_{n \gamma}\right\|_{L^{q}}=0$ and $\lim _{n \rightarrow \infty}\left|v_{n \gamma, s}\right|=0$. Therefore the bundle map $h_{n \gamma}: E_{n \gamma}=E_{\gamma} \rightarrow E_{\gamma}$ given in (34) can be defined for $n \geq n_{1}(\gamma)$. Here $n_{1}(\gamma)$ is an appropriate large number with $n_{1}(\gamma) \geq n_{0}(\gamma), n_{0}\left(\gamma s^{-1}\right)(s \in S)$. For each $n \geq 1$ there exist a (possibly empty) finite subset $\Omega_{n} \subset \Gamma$ (each $\gamma \in \Omega_{n}$ satisfies $n \geq n_{1}(\gamma)$ ) such that we can define a bundle map $h_{n}:\left.\left.\boldsymbol{E}_{n}\right|_{X_{\Omega_{n}}} \rightarrow \boldsymbol{E}\right|_{X_{\Omega_{n}}}$ by gluing these $h_{n \gamma}$. We can take these $\Omega_{n}$ so that $\Omega_{1} \subset \Omega_{2} \subset \Omega_{3} \subset \cdots$ and $\bigcup_{n \geq 1} \Omega_{n}=\Gamma$.

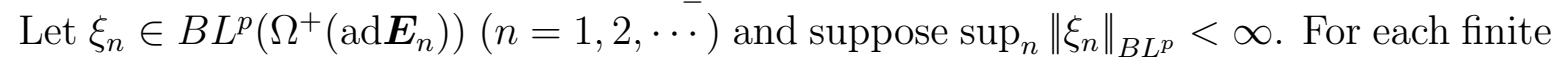
subset $\Omega \subset \Gamma, L^{p}\left(X_{\Omega}\right)$ is a reflexive Banach space. Hence if we take a subsequence of $\left\{\xi_{n}\right\}$, there exists $\xi \in B L^{p}\left(\Omega^{+}(\operatorname{ad} \boldsymbol{E})\right)$ with $\|\xi\|_{B L^{p}} \leq \sup _{n}\left\|\xi_{n}\right\|_{B L^{p}}$ such that, for any finite subset $\Omega \subset \Gamma,\left.h_{n}\left(\xi_{n}\right)\right|_{\Omega}$ weakly converges to $\left.\xi\right|_{\Omega}$ in $L^{p}\left(X_{\Omega}\right)$.

Lemma 6.9. In the above situation, $\left.h_{n}\left(P_{\theta_{n}}\left(\xi_{n}\right)\right)\right|_{X_{\Omega}}$ weakly converges to $\left.P_{\theta}(\xi)\right|_{X_{\Omega}}$ in $L^{q}\left(X_{\Omega}\right)$ as $n \rightarrow \infty$ for any finite subset $\Omega \subset \Gamma$.

Proof. Take $\varepsilon>0$ and $\eta \in\left(L^{q}\left(X_{\Omega}\right)\right)^{*}=L^{q^{\prime}}\left(X_{\Omega}\right)\left(1 / q+1 / q^{\prime}=1\right)$. Let $d>0$ be a large integer which will be fixed later. Set $\xi_{n}^{\prime}:=\left.\xi_{n}\right|_{B_{d}(\Omega)}=1_{X_{B_{d}(\Omega)}} \cdot \xi_{n}$ and $\xi_{n}^{\prime \prime}:=\xi_{n}-\xi_{n}^{\prime}$ where $1_{X_{B_{d}(\Omega)}}$ is the characteristic function of $X_{B_{d}(\Omega)}$. We also define $\xi^{\prime}:=\left.\xi\right|_{B_{d}(\Omega)}$ and $\xi^{\prime \prime}:=\xi-\xi^{\prime}$. $h_{n}\left(\xi_{n}^{\prime}\right)$ weakly converges to $\xi^{\prime}$ in $L^{p}\left(X_{B_{d}(\Omega)}\right)$. Then $P\left(h_{n}\left(\xi_{n}^{\prime}\right)\right)$ weakly converges to $P\left(\xi^{\prime}\right)$ in $B L^{q}$. Set $P_{n}^{\prime}:=h_{n} \circ P_{n} \circ h_{n}^{-1}: L^{p}\left(X_{B_{d}(\Omega)}, \Omega^{+}(\operatorname{ad} \boldsymbol{E})\right) \rightarrow B L^{q} .\left(P_{n}:=P_{\theta_{n}}.\right)$ We have

$$
h_{n}\left(P_{n}\left(\xi_{n}\right)\right)=\left(P_{n}^{\prime}\left(h_{n} \xi_{n}^{\prime}\right)-P\left(h_{n} \xi_{n}^{\prime}\right)\right)+P\left(h_{n} \xi_{n}^{\prime}\right)+h_{n}\left(P_{n}\left(\xi_{n}^{\prime \prime}\right)\right) .
$$

From Lemma 6.7, $\left\|h_{n}\left(P_{n}\left(\xi_{n}^{\prime \prime}\right)\right)\right\|_{L^{q}\left(X_{\Omega}\right)} \leq \operatorname{const}_{\Omega, M} \cdot 2^{-d}\left\|\xi_{n}\right\|_{B L^{p}}$ and $\left\|P\left(\xi^{\prime \prime}\right)\right\|_{L^{q}\left(X_{\Omega}\right)} \leq$ const $_{\Omega, M}$. $2^{-d}\|\xi\|_{B L^{p}} \leq \operatorname{const}_{\Omega, M} \cdot 2^{-d} \sup _{n}\left\|\xi_{n}\right\|_{B L^{p}}$.

The term $\left(P_{n}^{\prime}\left(h_{n} \xi_{n}^{\prime}\right)-P\left(h_{n} \xi_{n}^{\prime}\right)\right)$ can be evaluated by using Lemma 5.1 and 6.7 as follows. Define (for $n \gg 0)$ a gluing data $\hat{\theta}_{n}=\left(\hat{E}_{n \gamma}, \hat{A}_{n \gamma}, \hat{\rho}_{n \gamma, s}\right)_{\gamma \in \Gamma, s \in S}$ by $\left(\hat{E}_{n \gamma}, \hat{A}_{n \gamma}, \hat{\rho}_{n \gamma, s}\right)=$ $\left(E_{n \gamma}, A_{n \gamma}, \rho_{n \gamma, s}\right)$ for $(\gamma, s) \in B_{d+1}(\Omega) \times S$ and $\left(\hat{E}_{n \gamma}, \hat{A}_{n \gamma}, \hat{\rho}_{n \gamma, s}\right)=\left(E_{\gamma}, A_{\gamma}, \rho_{\gamma, s}\right)$ otherwise. Lemma 6.7 gives $\left(\hat{P}_{n}^{\prime}:=h_{n} \circ P_{\hat{\theta}_{n}} \circ h_{n}^{-1}\right)$

$$
\left\|P_{n}^{\prime}\left(h_{n} \xi_{n}^{\prime}\right)-\hat{P}_{n}^{\prime}\left(h_{n} \xi_{n}^{\prime}\right)\right\|_{L^{q}\left(X_{\Omega}, g\right)} \leq \operatorname{const}_{\Omega, M} \cdot 2^{-d}\left\|\xi_{n}\right\|_{B L^{p}}
$$

Lemma 5.1 gives $(n \gg 1)$

$\left\|\hat{P}_{n}^{\prime}\left(h_{n} \xi_{n}^{\prime}\right)-P\left(h_{n} \xi_{n}^{\prime}\right)\right\|_{B L^{q}} \leq \operatorname{const}_{M} \cdot \sup _{\gamma \in B_{d+1}(\Omega), s \in S}\left\{d_{L^{q}}\left(\left[A_{n \gamma}\right],\left[A_{\gamma}\right]\right)+\left|\rho_{n \gamma, s}-\rho_{\gamma, s}\right|\right\}\left\|\xi_{n}\right\|_{B L^{p}}$

Therefore for $\eta \in\left(L^{q}\left(X_{\Omega}\right)\right)^{*}$

$$
\begin{array}{r}
\left|\left\langle h_{n}\left(P_{n}\left(\xi_{n}\right)\right)-P(\xi), \eta\right\rangle\right| \leq\left|\left\langle P\left(h_{n} \xi_{n}^{\prime}\right)-P\left(\xi^{\prime}\right), \eta\right\rangle\right|+\text { const }_{\Omega, M} \cdot\|\eta\| 2^{-d} \sup _{m}\left\|\xi_{m}\right\|_{B L^{p}}, \\
\quad+\operatorname{const}_{\Omega, M} \cdot\|\eta\| \sup _{\gamma \in B_{d+1}(\Omega), s \in S}\left\{d_{L^{q}}\left(\left[A_{n \gamma}\right],\left[A_{\gamma}\right]\right)+\left|\rho_{n \gamma, s}-\rho_{\gamma, s}\right|\right\} \sup _{m}\left\|\xi_{m}\right\|_{B L^{p}}
\end{array}
$$

We choose $d>0$ so that const $_{\Omega, M} \cdot\|\eta\| 2^{-d} \sup _{n}\left\|\xi_{n}\right\|_{B L^{p}} \leq \varepsilon / 3$. We can choose $n_{1}>0$ so that for $n \geq n_{1}$

$$
\operatorname{const}_{\Omega, M} \cdot\|\eta\| \sup _{\gamma \in B_{d+1}(\Omega), s \in S}\left\{d_{L^{q}}\left(\left[A_{n \gamma}\right],\left[A_{\gamma}\right]\right)+\left|\rho_{n \gamma, s}-\rho_{\gamma, s}\right|\right\} \cdot \sup _{m}\left\|\xi_{m}\right\|_{B L^{p}} \leq \varepsilon / 3 .
$$


Since $P\left(h_{n} \xi_{n}^{\prime}\right)$ weakly converges to $P\left(\xi^{\prime}\right)$ in $B L^{q}$, we can choose $n_{2}$ so that for $n \geq n_{2}$

$$
\left|\left\langle P\left(h_{n} \xi_{n}^{\prime}\right)-P\left(\xi^{\prime}\right), \eta\right\rangle\right| \leq \varepsilon / 3 .
$$

Therefore for $n \geq \max \left(n_{1}, n_{2}\right)$

$$
\left|\left\langle h_{n}\left(P_{n}\left(\xi_{n}\right)\right)-P(\xi), \eta\right\rangle\right| \leq \varepsilon .
$$

Thus $\lim _{n \rightarrow \infty}\left\langle h_{n}\left(P_{n}\left(\xi_{n}\right)\right), \eta\right\rangle=\langle P(\xi), \eta\rangle$. This means that $\left.h_{n}\left(P_{n}\left(\xi_{n}\right)\right)\right|_{\Omega}$ weakly converges to $\left.P(\xi)\right|_{\Omega}$ in $L^{q}\left(X_{\Omega}\right)$.

6.3. Proof of Surjectivity. Let $\left(E_{1}, A_{1}\right)$ and $\left(E_{2}, A_{2}\right)$ be two pairs of a principal $S U(2)$ bundle over $X$ and an ASD connection on it. We define the $L^{q}$-distance between their gauge equivalence classes by (recall $q>4$ )

$$
d_{L^{q}}\left(\left[E_{1}, A_{1}\right],\left[E_{2}, A_{2}\right]\right):=\inf _{g: E_{1} \rightarrow E_{2}}\left\|A_{2}-g\left(A_{1}\right)\right\|_{L^{q}(X)},
$$

where $g$ runs over bundle isomorphisms between $E_{1}$ and $E_{2}$. If $E_{1}$ and $E_{2}$ are not isomorphic, then we set $d_{L^{q}}\left(\left[E_{1}, A_{1}\right],\left[E_{2}, A_{2}\right]\right):=\infty$. Recall that $M$ denotes a set of gauge equivalence classes of $(E, A)$ satisfying the conditions (a), (b), (c) in the beginning of Section 3.2. Let $\mathcal{L} \subset M$ be a subset such that there exists $\delta>0$ satisfying $B_{\delta}(\mathcal{L}) \subset M$. Here $B_{\delta}(\mathcal{L}) \subset M$ means that if a pair $(E, A)$ of a principal $S U(2)$-bundle $E$ over $X$ and an ASD connection $A$ on $E$ satisfies $d_{L^{q}}([E, A],[F, B]) \leq \delta$ for some $[F, B] \in \mathcal{L}$ then $[E, A] \in M$. We define $\operatorname{GlD}(\mathcal{L}) \subset$ GlD by

$$
\operatorname{GlD}(\mathcal{L}):=\left\{\left[\left(E_{\gamma}, A_{\gamma}, \rho_{\gamma, s}\right)_{\gamma \in \Gamma, s \in S}\right] \in \operatorname{GlD} \mid\left[E_{\gamma}, A_{\gamma}\right] \in \mathcal{L} \text { for all } \gamma \in \Gamma\right\}
$$

Let $\mathcal{B}$ be the set of all gauge equivalence classes of $(F, B)$ where $F$ is a principal $S U(2)$ bundle over $X^{\sharp(\Gamma, S)}$ and $B$ is a connection on it. By using the cut-off construction in Section 3.3, we have the map:

$$
J: \mathrm{GlD} \rightarrow \mathcal{B}, \quad[\theta] \mapsto\left[\boldsymbol{E}(\theta), \boldsymbol{A}^{\prime}(\theta)\right] .
$$

For $\left[F_{i}, B_{i}\right] \in \mathcal{B}(i=1,2)$, we define their $B L^{q}$-distance by

$$
d_{B L^{q}}\left(\left[F_{1}, B_{1}\right],\left[F_{2}, B_{2}\right]\right):=\inf _{g: F_{1} \rightarrow F_{2}}\left\|B_{2}-g\left(B_{1}\right)\right\|_{B L^{q}} .
$$

(This may be $+\infty$.) For $\nu>0$ we define a subset $U(\mathcal{L}, \nu) \subset \mathcal{B}$ by

$$
U(\mathcal{L}, \nu):=\left\{[F, B] \in \mathcal{B} \mid d_{B L^{q}}([F, B], J(\operatorname{GlD}(\mathcal{L})))<\nu,\left\|F_{B}^{+}\right\|_{B L^{p}}<\nu^{3 / 2}\right\} .
$$

Here $d_{B L^{q}}([F, B], J(\operatorname{GlD}(\mathcal{L})))<\nu$ means that there exists a gluing data $[\theta] \in \operatorname{GlD}(\mathcal{L})$ such that $d_{B L^{q}}\left([F, B],\left[\boldsymbol{E}(\theta), \boldsymbol{A}^{\prime}(\theta)\right]\right)<\nu$. The following lemma will be used in Section 8 . (This is essentially given in Donaldson-Kronheimer [5, Lemma (7.2.43)].)

Lemma 6.10. There exists $b_{0}=b_{0}(M, \nu)$ and $\nu^{\prime}=\nu^{\prime}(\nu)$ such that if $b=N \sqrt{\lambda} \leq b_{0}$ and $[F, B] \in \mathcal{B}$ satisfies for all $\gamma \in \Gamma$

$$
\inf _{[E, A] \in \mathcal{L}} d_{L^{q}}\left(\left[\left.F\right|_{X_{\gamma}^{\prime \prime}},\left.B\right|_{X_{\gamma}^{\prime \prime}}\right],\left[\left.E\right|_{X_{\gamma}^{\prime \prime}},\left.A\right|_{X_{\gamma}^{\prime \prime}}\right]\right)<\nu^{\prime}, \quad\left\|F_{B}^{+}\right\|_{B L^{p}}<\nu^{3 / 2}
$$


then we have $[F, B] \in U(\mathcal{L}, \nu)$

Proof. There are $\left[E_{\gamma}, A_{\gamma}\right] \in M(\gamma \in \Gamma)$ and bundle maps $g_{\gamma}:\left.\left.F\right|_{X_{\gamma}^{\prime \prime}} \rightarrow E_{\gamma}\right|_{X_{\gamma}^{\prime \prime}}$ such that

$$
\left\|g_{\gamma}(B)-A_{\gamma}\right\|_{L^{q}\left(X_{\gamma}^{\prime \prime}, g\right)}<\nu^{\prime}
$$

From (14) we get

$$
\left\|A_{\gamma}-A_{\gamma}^{\prime}\right\|_{L^{q}\left(X_{\gamma}^{\prime \prime}, g\right)} \leq \operatorname{const}_{M} \cdot b^{1+4 / q}
$$

Hence

$$
\left\|g_{\gamma}(B)-A_{\gamma}^{\prime}\right\|_{L^{q}\left(X_{\gamma}^{\prime \prime}, g\right)}<\nu^{\prime}+\operatorname{const}_{M} \cdot b^{1+4 / q} .
$$

For each $\gamma \in \Gamma$ and $s \in S$, we set $h_{\gamma, s}:=g_{\gamma s} g_{\gamma}^{-1}: E_{\gamma} \rightarrow E_{\gamma s}$ over the "neck" $W_{\gamma, s}:=$ $X_{\gamma}^{\prime \prime} \cap X_{\gamma s}^{\prime \prime}$ Then $\left\|h_{\gamma, s}\left(A_{\gamma}^{\prime}\right)-A_{\gamma s}^{\prime}\right\|_{L^{q}\left(W_{\gamma, s}, g\right)} \leq 2\left(\nu^{\prime}+\operatorname{const}_{M} \cdot b^{1+4 / q}\right)=: \varepsilon$. In the exponential gauges of $A_{\gamma}$ around $x_{\gamma, s}$ and $y_{\gamma, s}$, the connection matrix $A_{\gamma}^{\prime}=0$ over the necks. Therefore, in these gauges, $\left\|d h_{\gamma, s}\right\|_{L^{q}\left(W_{\gamma, s}, g\right)} \leq \varepsilon$. Using the Sobolev embedding $L_{1}^{q} \hookrightarrow C^{0,1-4 / q}$, we get

$$
\left|h_{\gamma, s}(x)-h_{\gamma, s}(y)\right| \leq \mathrm{const} \cdot \varepsilon|x-y|^{1-4 / q},
$$

for any $x, y \in W_{\gamma, s}$. (The above "const" does not depend on $\lambda$.) Since the right-hand-side is sufficiently small, there is $\rho_{\gamma, s}:\left(E_{\gamma}\right)_{x_{\gamma, s}} \rightarrow\left(E_{\gamma s}\right)_{y_{\gamma s, s}}$ such that $h_{\gamma, s}=\rho_{\gamma, s} e^{u_{\gamma, s}}$ and $u_{\gamma, s}$ satisfies

$$
\left\|d u_{\gamma, s}\right\|_{L^{q}\left(W_{\gamma, s}, g\right)} \leq \mathrm{const} \cdot \varepsilon, \quad\left|u_{\gamma, s}\right| \leq \mathrm{const} \cdot \lambda^{1 / 2-2 / q} \varepsilon .
$$

Set $\theta:=\left(E_{\gamma}, A_{\gamma}, \rho_{\gamma, s}\right)_{\gamma, s}$.

We define $k_{\gamma}:\left.\left.E_{\gamma}\right|_{X_{\gamma}^{\prime \prime}} \rightarrow E_{\gamma}\right|_{X_{\gamma}^{\prime \prime}}$ as follows; $k_{\gamma}$ is equal to $e^{\left(1-\beta_{\gamma}^{\prime}\right) u_{\gamma, s}}$ around the points $x_{\gamma, s}$, and equal to $e^{-\left(1-\beta_{\gamma}^{\prime}\right) \tilde{u}_{\gamma s^{-1, s}}}\left(\tilde{u}_{\gamma, s}=\rho_{\gamma s^{-1}, s} u_{\gamma s^{-1}, s} \rho_{\gamma s^{-1, s}}^{-1}\right)$ around the points $y_{\gamma, s} . k_{\gamma}$ is equal to 1 outside the "neck" regions. We set $\tilde{g}_{\gamma}:=k_{\gamma} g_{\gamma}:\left.\left.F\right|_{X_{\gamma}^{\prime \prime}} \rightarrow E_{\gamma}\right|_{X_{\gamma}^{\prime \prime}}$. These compatibly (i.e. $\left.\tilde{g}_{\gamma s}=\rho_{\gamma, s} \tilde{g}_{\gamma}\right)$ define $\tilde{g}: F \rightarrow \boldsymbol{E}(\theta)$. We have $\tilde{g}_{\gamma}(B)-A_{\gamma}^{\prime}=k_{\gamma}\left(g_{\gamma}(B)-\right.$ $\left.A_{\gamma}^{\prime}\right)+k_{\gamma}\left(A_{\gamma}^{\prime}\right)-A_{\gamma}^{\prime}$. From (61) we have $\left\|k_{\gamma}\left(A_{\gamma}^{\prime}\right)-A_{\gamma}^{\prime}\right\|_{L^{q}\left(W_{\gamma, s}, g\right)} \leq$ const $\cdot \varepsilon$. (Note that $\left|d \beta_{\gamma}^{\prime} \otimes u_{\gamma, s}\right| \leq$ const $\cdot \lambda^{-2 / q} \varepsilon$ and hence $\left\|d \beta_{\gamma}^{\prime} \otimes u_{\gamma, s}\right\|_{L^{q}\left(W_{\gamma, s}, g\right)} \leq$ const $\cdot \varepsilon$. $)$ Therefore we have

$$
\left\|\tilde{g}(B)-\boldsymbol{A}^{\prime}(\theta)\right\|_{B L^{q}} \leq \mathrm{const} \cdot \varepsilon
$$

Recall that $\mathcal{L} \subset M$ satisfies $B_{\delta}(\mathcal{L}) \subset M$ where $B_{\delta}(\mathcal{L})$ is the $\delta$-neighborhood of $\mathcal{L}$ with respect to the $L^{q}$-distance.

Theorem 6.11. There are $\nu_{0}(\delta)>0, N_{0}>0$ and $\lambda_{0}(N, \nu, \delta)>0$ satisfying the following: If $\nu \leq \nu_{0}(\delta), N \geq N_{0}$ and $\lambda \leq \lambda(N, \nu, \delta)$ then for any $[F, B] \in U(\mathcal{L}, \nu)$ there exist $[\theta] \in \mathrm{GlD}$ and $\xi \in B L^{p}\left(\Omega^{+}(\boldsymbol{E}(\theta))\right)$ satisfying

$$
[F, B]=\left[\boldsymbol{E}, \boldsymbol{A}^{\prime}(\theta)+P_{\theta}(\xi)\right], \quad\|\xi\|_{B L^{p}} \leq 3 \nu^{3 / 2} .
$$


In particular if $[F, B] \in U(\mathcal{L}, \nu)$ and $B$ is an $A S D$ connection, then there exists $[\theta] \in \mathrm{GID}$ satisfying $[F, B]=[\boldsymbol{E}(\theta), \boldsymbol{A}(\theta)]$ (see Proposition 4.3 and the statement of the uniqueness of $\boldsymbol{\xi}$ there).

We will prove this theorem by using the continuity method developed in DonaldsonKronheimer [5, Section 7.2.4, 7.2.5].

Let $[F, B] \in U(\mathcal{L}, \nu)$. There is $\left[F, \boldsymbol{B}^{\prime}\right] \in J(\operatorname{GlD}(\mathcal{L}))$ satisfying $B=\boldsymbol{B}^{\prime}+\boldsymbol{b}$ with $\|\boldsymbol{b}\|_{B L^{q}}<\nu$. For $t \in[0,1]$ we set $B_{t}:=\boldsymbol{B}^{\prime}+t \boldsymbol{b}$. For small $\nu>0$ and $b=4 N \sqrt{\lambda}>0$, all $B_{t}$ are contained in $U(\mathcal{L}, \nu)$; In fact, when $t=0,\left\|F^{+}\left(B_{0}\right)\right\|_{B L^{p}}=\left\|F^{+}\left(\boldsymbol{B}^{\prime}\right)\right\|_{B L^{p}} \leq$ const $_{M} \cdot b^{4 / p}<\nu^{3 / 2}$. For $t \in(0,1], F^{+}\left(B_{t}\right)=t F^{+}(B)+(1-t) F^{+}\left(\boldsymbol{B}^{\prime}\right)+\left(t^{2}-t\right)(\boldsymbol{b} \wedge \boldsymbol{b})^{+}$.

$$
\begin{aligned}
\left\|F^{+}\left(B_{t}\right)\right\|_{B L^{p}} & \leq t\left\|F^{+}(B)\right\|_{B L^{p}}+(1-t)\left\|F^{+}\left(\boldsymbol{B}^{\prime}\right)\right\|_{B L^{p}}+\text { const } \cdot\left(t-t^{2}\right)\|\boldsymbol{b}\|_{B L^{q}}^{2}, \\
& <t \cdot \nu^{3 / 2}+\operatorname{const}_{M} \cdot(1-t)\left(b^{4 / p}+\nu^{2}\right) \leq \nu^{3 / 2} .
\end{aligned}
$$

Hence $\left[F, B_{t}\right] \in U(\mathcal{L}, \nu)$.

Let $\varepsilon>0$ be a small number which will be fixed later. Let $S \subset[0,1]$ be the set of $t \in[0,1]$ such that there exist a gluing data $\theta_{t}, \xi_{t} \in B L^{p}\left(\Omega^{+}\left(\boldsymbol{E}\left(\theta_{t}\right)\right)\right)$ and a gauge transformation $u_{t}: F \rightarrow \boldsymbol{E}\left(\theta_{t}\right)$ satisfying

$$
u_{t}\left(B_{t}\right)=\boldsymbol{A}^{\prime}\left(\theta_{t}\right)+P_{\theta_{t}}\left(\xi_{t}\right), \quad\left\|\xi_{t}\right\|_{B L^{p}}<\varepsilon .
$$

We have $0 \in S$. From this equation, we have $u_{t}\left(F^{+}\left(B_{t}\right)\right)=F^{+}\left(\boldsymbol{A}^{\prime}\left(\theta_{t}\right)\right)+\xi_{t}+\left(P_{\theta_{t}} \xi_{t} \wedge\right.$ $\left.P_{\theta_{t}} \xi_{t}\right)^{+}$. Hence

$$
\begin{aligned}
\left\|\xi_{t}\right\|_{B L^{p}} & \leq\left\|F^{+}\left(B_{t}\right)\right\|_{B L^{p}}+\left\|F^{+}\left(\boldsymbol{A}^{\prime}\left(\theta_{t}\right)\right)\right\|_{B L^{p}}+\text { const }_{M} \cdot\left\|\xi_{t}\right\|_{B L^{p}}^{2} \\
& \leq \nu^{3 / 2}+\text { const }_{M}^{\prime} \cdot b^{4 / p}+\text { const }_{M} \cdot \varepsilon\left\|\xi_{t}\right\|_{B L^{p}} .
\end{aligned}
$$

We choose $\varepsilon>0$ so that $\operatorname{const}_{M} \cdot \varepsilon \leq 1 / 2$. Then

$$
\left\|\xi_{t}\right\|_{B L^{p}} \leq 2\left(\nu^{3 / 2}+\text { const }_{M}^{\prime} b^{4 / p}\right)
$$

We choose $\nu$ and $b$ sufficiently small so that $2\left(\nu^{3 / 2}+\right.$ const $\left._{M}^{\prime} b^{4 / p}\right) \leq 3 \nu^{3 / 2} \leq \varepsilon / 2$. Therefore we get

$$
\left\|\xi_{t}\right\|_{B L^{p}} \leq 3 \nu^{3 / 2} \leq \varepsilon / 2 .
$$

In particular, from the open condition $\left\|\xi_{t}\right\|_{B L^{p}}<\varepsilon$, we have deduced the closed condition $\left\|\xi_{t}\right\|_{B L^{p}} \leq \varepsilon / 2$.

Now we will prove that $S$ is a closed set in $[0,1]$. Let $t_{n} \in S(n=1,2,3, \cdots)$ be a sequence converging to $t \in[0,1]$. Set $\theta_{n}=\theta_{t_{n}}=\left(E_{n \gamma}, A_{n \gamma}, \rho_{n \gamma, s}\right)_{\gamma \in \Gamma, s \in S}$. We have $u_{n}\left(B_{t_{n}}\right)=\boldsymbol{A}_{n}^{\prime}+P_{n}\left(\xi_{n}\right)$ with $\left\|\xi_{n}\right\|_{B L^{p}} \leq \varepsilon / 2$. From the argument before Lemma 6.9, using some gauge transformations, we can suppose that $\theta_{n}$ converges to $\theta=\left(E_{\gamma}, A_{\gamma}, \rho_{\gamma, s}\right)_{\gamma \in \Gamma, s \in S}$ as follows; There is $n_{0}(\gamma)>0$ for each $\gamma \in \Gamma$ such that $E_{n \gamma}=E_{\gamma}, A_{n \gamma}=A_{\gamma}+\tilde{\alpha}_{n \gamma}\left(\alpha_{n \gamma} \in\right.$ $\left.H_{A_{\gamma}}^{1}\right), \rho_{n \gamma, s}=\rho_{\gamma, s} e^{v_{n \gamma, s}}$ for $n \geq n_{0}(\gamma)$, and $\alpha_{n \gamma}$ and $v_{n \gamma, s}$ converge to 0 as $n \rightarrow \infty$. Moreover there exist $\xi \in B L^{p}\left(\Omega^{+}(\operatorname{ad} \boldsymbol{E})\right)$, an exhausting sequence $\Omega_{1} \subset \Omega_{2} \subset \Omega_{3} \subset \cdots \subset \Gamma$ and bundle maps $h_{n}:\left.\left.\boldsymbol{E}_{n}\right|_{X_{\Omega_{n}}} \rightarrow \boldsymbol{E}\right|_{X_{\Omega_{n}}}$ such that $\left.h_{n}\left(\xi_{n}\right)\right|_{X_{\Omega}}$ weakly converges to $\left.\xi\right|_{X_{\Omega}}$ in 
$L^{p}\left(X_{\Omega}\right)$ for any finite subset $\Omega \subset \Gamma$. From $\left\|\xi_{n}\right\|_{B L^{p}} \leq \varepsilon / 2$, we have $\|\xi\|_{B L^{p}} \leq \varepsilon / 2$. Set $g_{n}:=h_{n} \circ u_{n}$ (over $X_{\Omega_{n}}$ ). Then

$$
g_{n}\left(B_{n}\right)=h_{n}\left(\boldsymbol{A}_{n}^{\prime}\right)+h_{n}\left(P_{n}\left(\xi_{n}\right)\right) \text { over } X_{\Omega_{n}} .
$$

For any finite subset $\Omega \subset \Gamma$, the right-hand-side of this equation weakly converges to $\boldsymbol{A}^{\prime}(\theta)+P_{\theta}(\xi)$ in $L^{q}\left(X_{\Omega}\right)$ (Lemma 6.9). On the other hand, if we take a subsequence, there exists a bundle map $g$ defined over $X^{\sharp(\Gamma, S)}$ such that $g_{n}$ weakly converges to $g$ in $L_{1}^{q}\left(X_{\Omega}\right)$ for each finite subset $\Omega \subset \Gamma$. Then we get

$$
g\left(B_{t}\right)=\boldsymbol{A}^{\prime}(\theta)+P_{\theta}(\xi), \quad\|\xi\|_{B L^{p}} \leq \varepsilon / 2<\varepsilon .
$$

This shows $t \in S$. Thus $S$ is a closed set in $[0,1]$.

Next we will prove that $S$ is open in $[0,1]$. Suppose that the equation (62) holds at some $t \in[0,1]$. Then $\boldsymbol{A}^{\prime}=\boldsymbol{A}^{\prime}\left(\theta_{t}\right)$ satisfies $d_{B L^{q}}\left(\left[\boldsymbol{A}^{\prime}\right],\left[\boldsymbol{B}^{\prime}\right]\right) \leq \nu+$ const $\cdot \varepsilon$. Therefore if we choose $b, \varepsilon$ and $\nu$ small enough, then $\theta_{t}=\left(E_{\gamma}, A_{\gamma}, \rho_{\gamma, s}\right)_{\gamma \in \Gamma, s \in S}$ satisfies

$$
B_{\delta / 2}\left(\left[E_{\gamma}, A_{\gamma}\right]\right) \subset M,
$$

for every $\gamma \in \Gamma$. (Recall that $B_{\delta}(\mathcal{L}) \subset M$.)

Consider the following map:

$$
G: B_{1} \rightarrow B_{2}, \quad(\chi, \boldsymbol{v}, \boldsymbol{\alpha}, \eta) \mapsto e^{-\chi}\left(\boldsymbol{A}_{\boldsymbol{v}, \boldsymbol{\alpha}}^{\prime}+P_{\boldsymbol{v}, \boldsymbol{\alpha}}\left(\eta+\xi_{t}\right)\right)-u_{t}\left(B_{t}\right),
$$

where $B_{1}$ and $B_{2}$ denote the Banach spaces defined in Section 6.1. Of course, we consider only very small $(\chi, \boldsymbol{v}, \boldsymbol{\alpha}, \eta) \in B_{1} . \boldsymbol{A}_{\boldsymbol{v}, \boldsymbol{\alpha}}^{\prime}$ and $P_{\boldsymbol{v}, \boldsymbol{\alpha}}$ is the connection and the operator defined in Section 5.1. $\boldsymbol{A}_{0,0}^{\prime}=\boldsymbol{A}^{\prime}=\boldsymbol{A}^{\prime}\left(\theta_{t}\right)$ and $P_{0,0}=P_{\theta_{t}}$. We have $G(0)=0$. If we prove that the derivative of $G$ at the origin $(d G)_{0}: B_{1} \rightarrow B_{2}$ is isomorphic, then the inverse mapping theorem and (64) implies that $t \in S$ is an inner point. $(d G)_{0}: B_{1} \rightarrow B_{2}$ is given by

$$
(d G)_{0}(\chi, \boldsymbol{v}, \boldsymbol{\alpha}, \eta)=T(\chi, \boldsymbol{v}, \boldsymbol{\alpha}, \eta)+\left[P\left(\xi_{t}\right), \chi\right]+\partial_{\boldsymbol{v}} P_{\boldsymbol{v}, 0}\left(\xi_{t}\right)+\partial_{\boldsymbol{\alpha}} P_{0, \boldsymbol{\alpha}}\left(\xi_{t}\right),
$$

where $T(\chi, \boldsymbol{v}, \boldsymbol{\alpha}, \eta)=d_{\boldsymbol{A}^{\prime}} \chi+j_{1}(\boldsymbol{v})+j_{2}(\boldsymbol{\alpha})+P(\eta)$ is the operator given in (48) and $P=$ $P_{0,0}=P_{\theta_{t}}$. Proposition 6.6 shows that $T$ is an isomorphism satisfying $\|(\chi, \boldsymbol{v}, \boldsymbol{\alpha}, \eta)\|_{B_{1}} \leq$ $K\|T(\chi, \boldsymbol{v}, \boldsymbol{\alpha}, \eta)\|_{B_{2}}$. Therefore if we prove $\left\|T-(d G)_{0}\right\|<K^{-1}$, then $(d G)_{0}$ is an isomorphism.

We have $\left\|\left[P\left(\xi_{t}\right), \chi\right]\right\|_{B L^{q}} \leq \operatorname{const}_{M} \cdot\left\|\xi_{t}\right\|_{B L^{p}}\|\chi\|_{\mathcal{C}^{0}} \leq \operatorname{const}_{M} \cdot \varepsilon\|(\chi, \boldsymbol{v}, \boldsymbol{\alpha}, \eta)\|_{B_{1}}$ from Lemma 6.2 and (46). We have $d_{\boldsymbol{A}^{\prime}}^{+}\left[P\left(\xi_{t}\right), \chi\right]=\left[\xi_{t}, \chi\right]-\left[P\left(\xi_{t}\right) \wedge d_{\boldsymbol{A}^{\prime}} \chi\right]^{+}$. Hence

$$
\left\|d_{\boldsymbol{A}^{\prime}}^{+}\left[P\left(\xi_{t}\right), \chi\right]\right\|_{B L^{p}} \leq\|\chi\|_{\mathcal{C}^{0}}\left\|\xi_{t}\right\|_{B L^{p}}+\left\|P\left(\xi_{t}\right)\right\|_{B L^{q}}\left\|d_{\boldsymbol{A}^{\prime}} \chi\right\|_{B L^{4}} .
$$

We have $\left\|d_{\boldsymbol{A}^{\prime}} \chi\right\|_{B L^{4}} \leq\left\|d_{\boldsymbol{A}^{\prime}} \chi+j_{1}(\boldsymbol{v})+j_{2}(\boldsymbol{\alpha})\right\|_{B L^{4}}+\left\|j_{1}(\boldsymbol{v})\right\|_{B L^{4}}+\left\|j_{2}(\boldsymbol{\alpha})\right\|_{B L^{4}}$. Recall the equation (45) $\left(j_{1}(\boldsymbol{v})=d \beta_{\gamma}^{\prime} \otimes v_{\gamma, s}\right.$ over $\left.\Omega\left(x_{\gamma, s}\right)\right)$ and the fact that $\left\|d \beta_{\gamma}^{\prime}\right\|_{L^{4}}$ is a constant independent of the parameters $\lambda$ and $N$ (because of the conformal invariance of the $L^{4}$ norms of 1-forms; see the argument before (17)). Hence (using Lemma 6.2)

$$
\left\|j_{1}(\boldsymbol{v})\right\|_{B L^{4}} \leq \mathrm{const}\|\boldsymbol{v}\| \leq \text { const }_{M}\|(\chi, \boldsymbol{v}, \boldsymbol{\alpha}, \eta)\|_{B_{1}} .
$$


From Lemma 6.1, $\left\|j_{2}(\boldsymbol{\alpha})\right\|_{B L^{4}} \leq$ const $\cdot\left\|j_{2}(\boldsymbol{\alpha})\right\|_{B L^{q}} \leq \operatorname{const}_{M} \cdot\|\boldsymbol{\alpha}\| \leq \operatorname{const}_{M} \cdot\|(\chi, \boldsymbol{v}, \boldsymbol{\alpha}, \eta)\|_{B_{1}}$. Hence $\left\|d_{\boldsymbol{A}^{\prime}}^{+}\left[P\left(\xi_{t}\right), \chi\right]\right\|_{B L^{p}} \leq \operatorname{const}_{M} \cdot \varepsilon\|(\chi, \boldsymbol{v}, \boldsymbol{\alpha}, \eta)\|_{B_{1}}$. Thus $\left\|\left[P\left(\xi_{t}\right), \chi\right]\right\|_{B_{2}} \leq$ const $_{M}$. $\varepsilon\|(\chi, \boldsymbol{v}, \boldsymbol{\alpha}, \eta)\|_{B_{1}}$.

By using an argument similar to that in Lemma 5.1, we have

$$
\left\|\partial_{\boldsymbol{v}} P_{\boldsymbol{v}, 0}\left(\xi_{t}\right)\right\|_{B L^{q}}+\left\|\partial_{\boldsymbol{\alpha}} P_{0, \boldsymbol{\alpha}}\left(\xi_{t}\right)\right\|_{B L^{q}} \leq \operatorname{const}_{M} \cdot \varepsilon(\|\boldsymbol{v}\|+\|\boldsymbol{\alpha}\|) \leq \operatorname{const}_{M} \cdot \varepsilon\|(\chi, \boldsymbol{v}, \boldsymbol{\alpha}, \eta)\|_{B_{1}} .
$$

Differentiating the equation $d_{\boldsymbol{A}_{\boldsymbol{v}, \boldsymbol{\alpha}}^{\prime}}^{+} P_{\boldsymbol{v}, \boldsymbol{\alpha}}\left(\xi_{t}\right)=\xi_{t}$ with respect to $\boldsymbol{v}$, we have $d_{\boldsymbol{A}^{\prime}}^{+} \partial_{\boldsymbol{v}} P_{\boldsymbol{v}, 0}\left(\xi_{t}\right)=$ $-\left(j_{1}(\boldsymbol{v}) \wedge P\left(\xi_{t}\right)\right)^{+}$. Using the above (65), we have

$$
\left\|d_{\boldsymbol{A}^{\prime}}^{+} \partial_{\boldsymbol{v}} P_{\boldsymbol{v}, 0}\left(\xi_{t}\right)\right\|_{B L^{p}} \leq\left\|j_{1}(\boldsymbol{v})\right\|_{B L^{4}}\left\|P\left(\xi_{t}\right)\right\|_{B L^{q}} \leq \operatorname{const}_{M} \cdot \varepsilon\|(\chi, \boldsymbol{v}, \boldsymbol{\alpha}, \eta)\|_{B_{1}} .
$$

Similarly

$$
\left\|d_{\boldsymbol{A}^{\prime}}^{+} \partial_{\boldsymbol{\alpha}} P_{0, \boldsymbol{\alpha}}\left(\xi_{t}\right)\right\|_{B L^{p}} \leq \operatorname{const}_{M} \cdot \varepsilon\|\boldsymbol{\alpha}\| \leq \operatorname{const}_{M} \cdot \varepsilon\|(\chi, \boldsymbol{v}, \boldsymbol{\alpha}, \eta)\|_{B_{1}} .
$$

Therefore

$$
\left\|\partial_{\boldsymbol{v}} P_{\boldsymbol{v}, 0}\left(\xi_{t}\right)\right\|_{B_{2}}+\left\|\partial_{\boldsymbol{\alpha}} P_{0, \boldsymbol{\alpha}}\left(\xi_{t}\right)\right\|_{B_{2}} \leq \operatorname{const}_{M} \cdot \varepsilon\|(\chi, \boldsymbol{v}, \boldsymbol{\alpha}, \eta)\|_{B_{1}}
$$

Thus

$$
\left\|(d G)_{0}(\chi, \boldsymbol{v}, \boldsymbol{\alpha}, \eta)-T(\chi, \boldsymbol{v}, \boldsymbol{\alpha}, \eta)\right\|_{B_{2}} \leq \operatorname{const}_{M} \cdot \varepsilon\|(\chi, \boldsymbol{v}, \boldsymbol{\alpha}, \eta)\|_{B_{1}} .
$$

Hence if we choose $\varepsilon$ small enough, then $(d G)_{0}$ is an isomorphism. This shows that $S$ is open in $[0,1] . S$ is a non-empty open closed set in $[0,1]$. Thus $S=[0,1]$. In particular we have $1 \in S$. This proves Theorem 6.11.

\section{Estimation of THE MEAN DIMENSION}

As in the previous sections, $M$ denotes a set of equivalence classes of $(E, A)$ ( $E$ is a principal $S U(2)$-bundle over $X$ and $A$ is an ASD connection on it) which satisfies the conditions (a), (b), (c) in the beginning of Section 3.2. GID $=\mathrm{GlD}_{M}$ is the set of the equivalence classes of $M$-gluing data defined in Definition 3.2. GID is endowed with the topology of "point-wise convergence" as follows. A sequence $\left[\theta_{n}\right]=\left[E_{n \gamma}, A_{n \gamma}, \rho_{n \gamma, s}\right]_{\gamma \in \Gamma, s \in S}$ in GID $(n \geq 1)$ converges to $[\theta]=\left[E_{\gamma}, A_{\gamma}, \rho_{\gamma, s}\right]_{\gamma \in \Gamma, s \in S}$ if the following condition is satisfied. For any finite subset $\Omega \subset \Gamma$, there exist $n_{0}(\Omega)>0$ and bundle isomorphisms $g_{n \gamma}: E_{n \gamma} \rightarrow$ $E_{\gamma}$ for $n \geq n_{0}(\Omega)$ and $\gamma \in \Omega$ such that $g_{n \gamma}\left(A_{n \gamma}\right)$ converges to $A_{\gamma}($ as $n \rightarrow \infty)$ in the $\mathcal{C}^{\infty}$ topology for all $\gamma \in \Omega$, and that $g_{n \gamma s} \rho_{n \gamma, s} g_{n \gamma}^{-1}:\left(E_{\gamma}\right)_{x_{\gamma, s}} \rightarrow\left(E_{\gamma s}\right)_{y_{\gamma s, s}}$ converges to $\rho_{\gamma, s}$ for any $(\gamma, s)$ with $\gamma, \gamma s \in \Omega$. This topology is metrizable and compact (because we suppose that $M$ is compact). $\Gamma$ continuously acts on GID by (this is a right action)

$$
\left[E_{\gamma}, A_{\gamma}, \rho_{\gamma, s}\right]_{\gamma \in \Gamma, s \in S} \cdot g:=\left[E_{g \gamma}, A_{g \gamma}, \rho_{g \gamma, s}\right]_{\gamma \in \Gamma, s \in S},
$$

where we naturally consider that $\left(E_{g \gamma}, A_{g \gamma}\right)$ is a data defined on $X_{\gamma}$ and that $\rho_{g \gamma, s}$ is a map from $\left(E_{g \gamma}\right)_{x_{\gamma, s}}$ to $\left(E_{g \gamma}\right)_{y_{\gamma s, s}}$. A distance on GID is given as follows: For $n \geq 1$, 
$[\theta]=\left[E_{\gamma}, A_{\gamma}, \rho_{\gamma, s}\right]_{\gamma \in \Gamma, s \in S}$ and $\left[\theta^{\prime}\right]=\left[F_{\gamma}, B_{\gamma}, \rho_{\gamma, s}^{\prime}\right]_{\gamma \in \Gamma, s \in S}$ in GID, we define $\delta_{n}\left([\theta],\left[\theta^{\prime}\right]\right)$ by

$$
\delta_{n}\left([\theta],\left[\theta^{\prime}\right]\right):=\inf _{g_{\gamma}: E_{\gamma} \rightarrow F_{\gamma}\left(\gamma \in B_{n}\right)}\left(\sum_{\gamma \in B_{n}}\left\|g_{\gamma}\left(A_{\gamma}\right)-B_{\gamma}\right\|_{L^{\infty}(X)}+\sum_{\gamma, \gamma s \in B_{n}}\left|g_{\gamma s} \rho_{\gamma, s} g_{\gamma}^{-1}-\rho_{\gamma, s}^{\prime}\right|\right),
$$

where $B_{n}$ is the $n$-ball (with respect to the word distance) centered at the origin in $\Gamma$ and $g_{\gamma}\left(\gamma \in B_{n}\right)$ runs over bundle isomorphisms between $E_{\gamma}$ and $F_{\gamma}$. If $E_{\gamma}$ is not isomorphic to $F_{\gamma}$ for some $\gamma \in B_{n}$, then we set $\delta_{n}\left([\theta],\left[\theta^{\prime}\right]\right):=+\infty$. We define a distance $d\left([\theta],\left[\theta^{\prime}\right]\right)$ by

$$
d\left([\theta],\left[\theta^{\prime}\right]\right):=\sum_{n \geq 1} \frac{1}{2^{n}} \frac{\delta_{n}\left([\theta],\left[\theta^{\prime}\right]\right)}{1+\delta_{n}\left([\theta],\left[\theta^{\prime}\right]\right)} .
$$

We define the space $\mathcal{M}(\mathrm{GlD})=\mathcal{M}\left(\mathrm{GlD}_{M}\right)$ by

$$
\mathcal{M}(\mathrm{GID}):=\{[\boldsymbol{E}(\theta), \boldsymbol{A}(\theta)] \mid[\theta] \in \mathrm{GID}\} .
$$

$\mathcal{M}(\mathrm{GlD})$ is endowed with the topology of $\mathcal{C}^{\infty}$-convergence on compact subsets in $X^{\sharp(\Gamma, S)}$. This topology is metrizable. $\Gamma$ continuously acts on $\mathcal{M}(\mathrm{GID})$ by (3). The map

$$
\mathrm{GlD} \rightarrow \mathcal{M}(\mathrm{GlD}), \quad[\theta] \mapsto[\boldsymbol{E}(\theta), \boldsymbol{A}(\theta)]
$$

is $\Gamma$-equivariant.

Lemma 7.1. The above map $\mathrm{GID} \rightarrow \mathcal{M}(\mathrm{GID})$ is a $\Gamma$-homeomorphism.

Proof. Proposition 5.5 shows that the map is bijective. Since GID is compact, it is enough to prove that the map is continuous.

Let $\varepsilon>0$ and $\Omega \subset \Gamma$ be a finite subset. Let $\theta_{1}=\left(E_{1 \gamma}, A_{1 \gamma}, \rho_{1 \gamma, s}\right)_{\gamma \in \Gamma, s \in S}$ and $\theta_{2}=$ $\left(E_{2 \gamma}, A_{2 \gamma}, \rho_{2 \gamma, s}\right)_{\gamma \in \Gamma, s \in S}$ be two gluing data. Let $d=d(M, \varepsilon)$ be the positive constant given by Corollary 6.8. Suppose that $E_{1 \gamma}=E_{2 \gamma}$ for $\gamma \in B_{d+1}(\Omega)$ and that $\left\|A_{1 \gamma}-A_{2 \gamma}\right\|_{L^{q}}$ and $\left|\rho_{1 \gamma, s}-\rho_{2 \gamma, s}\right|\left(\gamma \in B_{d}(\Omega), s \in S\right)$ are sufficiently small. We define another gluing data $\theta^{\prime}:=\left(E_{\gamma}^{\prime}, A_{\gamma}^{\prime}, \rho_{\gamma, s}^{\prime}\right)_{\gamma \in \Gamma, s \in S}$ by $\left(E_{\gamma}^{\prime}, A_{\gamma}^{\prime}, \rho_{\gamma, s}^{\prime}\right)=\left(E_{1 \gamma}, A_{1 \gamma}, \rho_{1 \gamma, s}\right)$ for $\gamma \in B_{d}(\Omega)$ and $\left(E_{\gamma}^{\prime}, A_{\gamma}^{\prime}, \rho_{\gamma, s}^{\prime}\right)=\left(E_{2 \gamma}, A_{2 \gamma}, \rho_{2 \gamma, s}\right)$ for $\gamma \in \Gamma \backslash B_{d}(\Omega)$. From Corollary 6.8, we have (for $\gamma \in \Omega)$

$$
\left\|\boldsymbol{A}\left(\theta_{1}\right)-\boldsymbol{A}\left(\theta^{\prime}\right)\right\|_{L^{q}\left(X_{\gamma}^{\prime \prime}, g\right)}<\varepsilon .
$$

On the other hand, for all $\gamma \in \Gamma$ and $s \in S$ we have $E_{2 \gamma}=E_{\gamma}^{\prime},\left\|A_{2 \gamma}-A_{\gamma}^{\prime}\right\|_{L^{q}\left(X_{\gamma}^{\prime \prime}, g\right)} \ll 1$ and $\left|\rho_{2 \gamma, s}-\rho_{\gamma}^{\prime}\right| \ll 1$. Therefore (using the arguments in Section 5.1)

$$
d_{B L^{q}}\left(\left[\boldsymbol{A}\left(\theta_{2}\right)\right],\left[\boldsymbol{A}\left(\theta^{\prime}\right)\right]\right)<\varepsilon
$$

Thus there exists a bundle map $g$ from $\boldsymbol{E}\left(\theta_{1}\right)$ to $\boldsymbol{E}\left(\theta_{2}\right)$ over $\Omega$ such that for all $\gamma \in \Omega$

$$
\left\|g\left(\boldsymbol{A}\left(\theta_{1}\right)\right)-\boldsymbol{A}\left(\theta_{2}\right)\right\|_{L^{q}\left(X_{\gamma}^{\prime \prime}, g\right)}<2 \varepsilon .
$$

This shows that $\mathrm{GlD} \rightarrow \mathcal{M}(\mathrm{GlD})$ is continuous. 
In the rest of this section we assume that $\Gamma$ is amenable. Let $\Omega_{1} \subset \Omega_{2} \subset \Omega_{3} \subset \cdots$ be an amenable sequence in $\Gamma$. This sequence satisfies (for any $r>0$ )

$$
\left|B_{r}\left(\Omega_{n}\right)\right| /\left|\Omega_{n}\right| \rightarrow 1 \quad(n \rightarrow \infty),
$$

where $B_{r}\left(\Omega_{n}\right)$ is the $r$-neighborhood of $\Omega_{n}$. For each $\Omega_{n}$ we define the distance $d_{\Omega_{n}}\left([\theta],\left[\theta^{\prime}\right]\right)$ on GID by

$$
d_{\Omega_{n}}\left([\theta],\left[\theta^{\prime}\right]\right):=\max _{g \in \Omega_{n}} d\left([\theta] . g,\left[\theta^{\prime}\right] . g\right)
$$

\section{Proposition 7.2.}

$$
\operatorname{dim}(\mathcal{M}(\mathrm{GlD}): \Gamma) \leq 3|S|+\operatorname{dim} M,
$$

where $\operatorname{dim} M$ denotes the (topological) covering dimension of $M$.

Proof. From Lemma 7.1, it is enough to prove that

$$
\operatorname{dim}(\mathrm{GlD}: \Gamma) \leq 3|S|+\operatorname{dim} M .
$$

Fix any $\varepsilon>0$. Take $n_{0}=n_{0}(\varepsilon)>0$ satisfying

$$
\sum_{n \geq n_{0}} \frac{1}{2^{n}}<\varepsilon .
$$

For any finite set $\Omega \subset \Gamma$, we define $B_{-1}(\Omega)$ as the set of $\gamma \in \Omega$ satisfying $\gamma s \in \Omega$ for all $s \in S$. We define a finite dimensional compact metrizable space $\left.\mathrm{GlD}\right|_{\Omega}$ by

$$
\left.\mathrm{GID}\right|_{\Omega}:=\left\{\left(\left(E_{\gamma}, A_{\gamma}\right)_{\gamma \in \Omega},\left(\rho_{\gamma, s}\right)_{\gamma \in B_{-1}(\Omega), s \in S}\right) \mid\left[E_{\gamma}, A_{\gamma}\right] \in M, \rho_{\gamma, s}:\left(E_{\gamma}\right)_{x_{s}} \rightarrow\left(E_{\gamma s}\right)_{y_{s}}\right\} / \sim,
$$

where the equivalence relation $\sim$ is defined as follows. $\theta=\left(\left(E_{\gamma}, A_{\gamma}\right)_{\gamma \in \Omega},\left(\rho_{\gamma, s}\right)_{\gamma \in B_{-1}(\Omega), s \in S}\right)$ is equivalent to $\theta^{\prime}=\left(\left(F_{\gamma}, B_{\gamma}\right)_{\gamma \in \Omega},\left(\rho_{\gamma, s}^{\prime}\right)_{\gamma \in B_{-1}(\Omega), s \in S}\right)$ if there exist $g_{\gamma}: E_{\gamma} \rightarrow F_{\gamma}\left(\gamma \in \Omega_{n}\right)$ such that $g_{\gamma}\left(A_{\gamma}\right)=B_{\gamma}$ for all $\gamma \in \Omega$ and $\rho_{\gamma, s}^{\prime} g_{\gamma}=g_{\gamma s} \rho_{\gamma, s}$ for all $(\gamma, s) \in B_{-1}\left(\Omega_{n}\right) \times S$.

There is a natural projection GID $\left.\rightarrow \mathrm{GID}\right|_{\Omega}$. Consider the following projection map:

$$
\left.\mathrm{GID}\right|_{\Omega} \rightarrow M^{\Omega}
$$

The topological dimension of each fiber of this map is $\leq 3|\Omega||S|$. Hence

$$
\left.\operatorname{dim} \mathrm{GlD}\right|_{\Omega} \leq|\Omega| \operatorname{dim} M+3|\Omega||S|
$$

For each $\Omega_{n}$ in the amenable sequence, consider the following map

$$
p:\left.\mathrm{GlD} \rightarrow \mathrm{GlD}\right|_{B_{n_{0}}\left(\Omega_{n}\right)} .
$$

If $p([\theta])=p\left(\left[\theta^{\prime}\right]\right)$, then we have

$$
d_{\Omega_{n}}\left([\theta],\left[\theta^{\prime}\right]\right)<\varepsilon \text {. }
$$

Therefore (see Appendix B)

$\operatorname{Widim}_{\varepsilon}\left(\operatorname{GlD}, d_{\Omega_{n}}\right) \leq \operatorname{dim}\left(\left.\operatorname{GlD}\right|_{B_{n_{0}}\left(\Omega_{n}\right)}\right) \leq\left|B_{n_{0}}\left(\Omega_{n}\right)\right|(\operatorname{dim} M+3|S|)$.

Using (66), we get

$$
\operatorname{Widim}_{\varepsilon}(\operatorname{GlD}: \Gamma)=\lim _{n \rightarrow \infty} \operatorname{Widim}_{\varepsilon}\left(\operatorname{GlD}, d_{\Omega_{n}}\right) /\left|\Omega_{n}\right| \leq \operatorname{dim} M+3|S| .
$$


This holds for any $\varepsilon>0$. Thus we get the above result.

Let $[E, A] \in M$. We call this point a regular point of $M$ if $[E, A] \in M_{0}$ and there is $\delta>0$ such that, for any ASD connection $B$ on $E$ satisfying $d_{L^{q}}([A],[B]) \leq \delta$, the pair $[E, B]$ is contained in $M_{0}$. Remember that for any $[E, A] \in M_{0}$ the connection $A$ is irreducible (see (b) in the beginning of Section 3.2) and then its isotropy group is $\{ \pm 1\}$.

Proposition 7.3. Let $[E, A]$ be a regular point of $M$, then we have

$$
\operatorname{dim}(\mathcal{M}(\mathrm{GlD}): \Gamma) \geq 3|S|+\operatorname{dim} H_{A}^{1}
$$

Proof. We will prove $\operatorname{dim}($ GID $: \Gamma) \geq 3|S|+\operatorname{dim} H_{A}^{1}$. There is a compact neighborhood $K$ of the origin in $H_{A}^{1}$ such that, for all $\alpha \in K,[E, A+\tilde{\alpha}] \in M_{0}$ and the map $K \ni \alpha \mapsto$ $[E, A+\tilde{\alpha}] \in M_{0}$ is injective. Here $A+\tilde{\alpha}$ is the ASD connection introduced in Section 3.4.2. Let $\operatorname{Hom}_{S U(2)}\left(E_{x_{s}}, E_{y_{s}}\right)$ be the space of $S U(2)$-isomorphisms between the fibers $E_{x_{s}}$ and $E_{y_{s}}(s \in S)$. Let $L_{s} \subset \operatorname{Hom}_{S U(2)}\left(E_{x_{s}}, E_{y_{s}}\right)$ be a compact set such that $L_{s}$ is homeomorphic to a three dimensional ball and that, for any $u, v \in L_{s}$, we have $u \neq-v$. Then we have a natural $\Gamma$-equivariant continuous map

$$
\left(K \times \prod_{s \in S} L_{s}\right)^{\Gamma} \rightarrow \text { GlD }
$$

From the conditions of $K$ and $L_{s}$, this map is injective. Therefore

$$
\operatorname{dim}(\mathrm{GlD}: \Gamma) \geq \operatorname{dim}\left(\left(K \times \prod_{s \in S} L_{s}\right)^{\Gamma}: \Gamma\right)=\operatorname{dim}\left(K \times \prod_{s \in S} L_{s}\right)=\operatorname{dim} H_{A}^{1}+3|S| .
$$

\section{Proof of Theorem 2.3}

In this and the next sections we set $X:=S^{4}$ with the metric $h$ satisfying the conditions (i), (ii) in the beginning of Section 2.1. Let $0 \leq c<\bar{c}<+\infty$ and $d \in(2,+\infty]$. As in Section 2.1 we define $M=M_{S^{4}}(\bar{c}, d)$ as the space of the gauge equivalence classes of $(E, A)$ where $E$ is a principal $S U(2)$-bundle over $S^{4}$ and $A$ is an ASD connection on it satisfying $\left\|F_{A}\right\|_{L^{d}\left(S^{4}, h\right)} \leq \bar{c}$. We set $M_{1}:=\left\{\left[S^{4} \times S U(2)\right.\right.$, the product connection $\left.]\right\}$ and $M_{0}:=M \backslash M_{1}$.

If $A$ is an ASD connection on $\left(S^{4}, h\right)$, then $A$ is also ASD with respect to the standard metric on $S^{4}$ because $h$ is conformally equivalent to the standard one. Therefore all nonflat ASD connections on $\left(S^{4}, h\right)$ are regular. In particular, $M=M_{0} \sqcup M_{1}$ satisfies the conditions (a), (b), (c) in the beginning of Section 3.2. We consider the gluing data space $\mathrm{GlD}=\mathrm{GlD}_{M}$ for this $M=M_{S^{4}}(\bar{c}, d)$. 
Recall that $\mathcal{M}(c, d)$ is the space of $[E, A]$ where $E$ is a principal $S U(2)$-bundle over $\left(S^{4}\right)^{\sharp(\Gamma, S)}$ and $A$ is an ASD connection on it satisfying (2):

$$
\left\|F_{A}\right\|_{L^{d}\left(X_{\gamma}^{\prime \prime}, g\right)} \leq c \quad \text { for all } \gamma \in \Gamma
$$

(Here $X_{\gamma}^{\prime \prime}=U_{\gamma}$ in the notation of Section 2.1.)

Proposition 8.1. There are $N_{0}(c, \bar{c}, d)>0$ and $\lambda_{0}(c, \bar{c}, d, N)>0$ such that if $N \geq$ $N_{0}(c, \bar{c}, d)$ and $\lambda \leq \lambda_{0}(c, \bar{c}, d, N)$ then

$$
\mathcal{M}(c, d) \subset \mathcal{M}(\mathrm{GlD}),
$$

i.e., for any $[E, A] \in \mathcal{M}(c, d)$ there exists $[\theta] \in \operatorname{GlD}$ satisfying $[E, A]=[\boldsymbol{E}(\theta), \boldsymbol{A}(\theta)]$.

We need some preliminary results for the proof of this proposition. Our argument is based on Donaldson-Kronheimer [5, Section 7.3]. The following proposition is given in [5, Proposition 7.3.3], and we omit the proof.

Proposition 8.2. Let $T>0$ and $k>0$. Consider $(-T, T) \times S^{3}$ with the usual product metric. There are positive constants $\eta$ and $C=C(k)$ ( $\eta$ is independent of $T$ and $k$, and $C$ is independent of $T$ ) such that if an $A S D$ connection $A$ on $(-T, T) \times S^{3}$ satisfies

$$
\|F(A)\|_{L^{2}}^{2}:=\int_{(-T, T) \times S^{3}}|F(A)|^{2} d \mathrm{vol} \leq \eta^{2},
$$

then

$$
|F(A)| \leq C e^{2(|t|-T)}\|F(A)\|_{L^{2}},
$$

for all $(t, \theta) \in(-T, T) \times S^{3}$ with $|t| \leq T-k$.

Using the stereographic projection, we can translate this proposition to a result on the Euclidean space:

Corollary 8.3. Let $\sigma>0$ and $\lambda>0$ with $\lambda / \sigma \leq \sqrt{\lambda} / 2$. Set $k:=0.9$ and

$$
\Omega:=\left\{x \in \mathbb{R}^{4}|k \lambda / \sigma<| x \mid<k^{-1} \sigma\right\} .
$$

There exist $\eta>0$ and $C>0$ (independent of $\sigma$ and $\lambda$ ) such that if an ASD connection $A$ on $\Omega$ satisfies

$$
\|F(A)\|_{L^{2}}^{2}:=\int_{\Omega}|F(A)|^{2} d \mathrm{vol} \leq \eta^{2}
$$

then

$$
|F(A)| \leq \frac{C}{\sigma^{2}}\|F(A)\|_{L^{2}} \quad(\sqrt{\lambda} / 2 \leq|x| \leq \sigma) .
$$

Moreover $A$ can be represented by the connection matrix satisfying

$$
|A| \leq \frac{C|x|}{\sigma^{2}}\|F(A)\|_{L^{2}} \quad(\sqrt{\lambda} / 2 \leq|x| \leq \sigma) .
$$

Proof. See Donaldson-Kronheimer [5, p. 314]. 
Lemma 8.4. For any $\nu>0$ there is $\lambda_{0}>0$ such that if $\lambda \leq \lambda_{0}$ then all $[E, B] \in \mathcal{M}(c, d)$ satisfies

$$
\inf _{[A] \in M_{S^{4}}(c, d)} d_{L^{q}}\left(\left[\left.B\right|_{X_{\gamma}^{\prime \prime}}\right],\left[\left.A\right|_{X_{\gamma}^{\prime \prime}}\right]\right)<\nu \quad \text { for all } \gamma \in \Gamma .
$$

Note that $[A]$ runs over $M_{S^{4}}(c, d)\left(\right.$ not $\left.M=M_{S^{4}}(\bar{c}, d)\right)$.

Proof. We can prove this lemma by using the argument in [5, Section 7.3.4]. For $\varepsilon>0$ we set (cf. Section 3.4.3)

$$
X_{\varepsilon}:=S^{4} \backslash\left(\bigcup_{s \in S} \bar{B}\left(x_{s}, \varepsilon\right) \cup \bar{B}\left(y_{s}, \varepsilon\right)\right) .
$$

Suppose the above statement is false. Then there are $\nu>0$ and a decreasing sequence $\lambda_{1}>\lambda_{2}>\lambda_{3}>\cdots \rightarrow 0$ and ASD connections $B_{n}$ on $X_{\lambda_{n} / \sigma}(\sigma$ is a small positive constant chosen below) satisfying

$$
\begin{gathered}
\left\|F\left(B_{n}\right)\right\|_{L^{d}\left(X_{\sqrt{\lambda_{n}} / 2}, g\right)} \leq c \\
\inf _{[A] \in M_{S^{4}}(c, d)} d_{L^{q}}\left(\left[\left.B_{n}\right|_{X_{\sqrt{\lambda_{n}} / 2}}\right],\left[\left.A\right|_{X_{\sqrt{\lambda_{n}} / 2}}\right]\right) \geq \nu .
\end{gathered}
$$

Let $\Omega_{n}^{\prime}\left(x_{s}\right)$ and $\Omega_{n}^{\prime}\left(y_{s}\right)(s \in S)$ be the annulus regions (in $\left.X\right)$ around $x_{s}$ and $y_{s}$ of inner radius $=k \lambda_{n} / \sigma$ and outer radius $=\sigma$. Since $d>2$, we can choose $\sigma>0$ so small that all $B_{n}$ and $[A] \in M_{S^{4}}(c, d)$ have curvatures of $L^{2}$-norm $\leq \eta$ over each $\Omega_{n}^{\prime}\left(x_{s}\right)$ and $\Omega_{n}^{\prime}\left(y_{s}\right)$. $(\eta$ is a positive constant given in Corollary 8.3.)

From (67) and $d>2$, the Uhlenbeck compactness implies that (if we choose a subsequence) there exists $[A] \in M_{S^{4}}(c, d)$ such that $\left[B_{n}\right]$ converges to $[A]$ in the $\mathcal{C}^{\infty}$-topology over compact subsets in $X \backslash\left\{x_{s}, y_{s} \mid s \in S\right\}$.

On the other hand, from Corollary 8.3, $B_{n}$ and $A$ can be represented over $\Omega_{n}^{\prime}\left(x_{s}\right)$ and $\Omega_{n}^{\prime}\left(y_{s}\right)$ by the connection matrices satisfying

$$
\left|B_{n}\right| \leq \text { const } \cdot|x|, \quad|A| \leq \text { const } \cdot|x| \quad\left(\sqrt{\lambda_{n}} / 2 \leq|x| \leq \sigma\right)
$$

where "const" is a positive constant independent of $\lambda_{n}$. This estimate and the $\mathcal{C}^{\infty}$ convergence mentioned above imply

$$
d_{L^{q}}\left(\left[\left.B_{n}\right|_{X_{\sqrt{\lambda_{n}} / 2}}\right],\left[\left.A\right|_{X_{\sqrt{\lambda_{n}} / 2}}\right]\right) \rightarrow 0 .
$$

This contradicts (68).

Proof of Proposition 8.1. Set $\mathcal{L}:=M_{S^{4}}(c, d) \Subset M=M_{S^{4}}(\bar{c}, d)$. For any $\nu>0$, Lemma 6.10 and Lemma 8.4 implies (for appropriate $N$ and $\lambda) \mathcal{M}(c, d) \subset U(\mathcal{L}, \nu)$. Then, from Theorem 6.11, for any $[E, A] \in \mathcal{M}(c, d)$ there exists $[\theta] \in \operatorname{GlD}_{M}$ satisfying $[E, A]=$ $[\boldsymbol{E}(\theta), \boldsymbol{A}(\theta)]$. 
Proof of Theorem 2.3. (i) Take $\bar{c}$ such that $0 \leq c<\bar{c}<c_{0}(d)$. Then $M:=M_{S^{4}}(\bar{c}, d)=M_{1}$ i.e., $M$ consists only of the product connection. Then all $\boldsymbol{A}(\theta)\left([\theta] \in \mathrm{GlD}_{M}\right)$ become flat connections. (See Remark 4.5.) Since we have $\mathcal{M}(c, d) \subset \mathcal{M}\left(\mathrm{GlD}_{M}\right)$ (for appropriate $N$ and $\lambda), \mathcal{M}(c, d)$ is equal to the moduli space of flat $S U(2)$-connections.

(ii) Fix $N=N_{0}(c, \bar{c}, d)$ (the constant in Proposition 8.1). Using Propositions 7.2 and 8.1, we get $(\lambda \ll 1)$

$$
\operatorname{dim}(\mathcal{M}(c, d): \Gamma) \leq \operatorname{dim}(\mathcal{M}(\mathrm{GlD}): \Gamma) \leq 3|S|+\operatorname{dim} M_{S^{4}}(\bar{c}, d)
$$

\section{Proof of Theorem 2.4}

In this section we suppose $X=S^{4}$ and $d \in(2,+\infty)$. Let $0<\underline{c}<c<+\infty$ and set $c^{\prime}:=(\underline{c}+c) / 2\left(\underline{c}<c^{\prime}<c\right)$. We also suppose that $\operatorname{dim} M_{S^{4}}(\underline{c}, d)>0$. Then there exists $\left[E_{0}, A_{0}\right] \in M_{S^{4}}(\underline{c}, d)$ such that $A_{0}$ is a regular ASD connection and $\operatorname{dim} H_{A_{0}}^{1} \geq$ $\operatorname{dim} M_{S^{4}}(\underline{c}, d) . \quad\left[E_{0}, A_{0}\right]$ becomes a regular point of $M^{\prime}:=M_{S^{4}}\left(c^{\prime}, d\right)$. (See Proposition 7.3.)

Proposition 9.1. There is $b_{0}\left(c, c^{\prime}, d\right)>0$ such that if $b=4 N \sqrt{\lambda} \leq b_{0}\left(c, c^{\prime}, d\right)$ then

$$
\mathcal{M}\left(\mathrm{GlD}_{M^{\prime}}\right) \subset \mathcal{M}(c, d),
$$

i.e., for any $[\theta] \in \mathrm{GlD}_{M^{\prime}}$ we have

$$
\|F(\boldsymbol{A}(\theta))\|_{L^{d}\left(X_{\gamma}^{\prime \prime}, g\right)} \leq c \quad \text { for all } \gamma \in \Gamma .
$$

Proof. We use an argument similar to that in the proof of Lemma 8.4. Set $\varepsilon:=\left(c-c^{\prime}\right) / 2=$ $(c-\underline{c}) / 4$. Suppose the above statement is false. Then there are parameters $\lambda_{n}$ and $N_{n} \geq N_{0}\left(M^{\prime}\right)(n=1,2,3, \cdots)\left(N_{0}\left(M^{\prime}\right)\right.$ is the constant given by Proposition 4.3) satisfying $b_{n}:=4 N_{n} \sqrt{\lambda_{n}} \rightarrow 0$, and $M^{\prime}$-gluing data $\theta_{n}=\left(E_{n \gamma}, A_{n \gamma}, \rho_{n \gamma, s}\right)_{\gamma \in \Gamma, s \in S}$ such that for some $\gamma_{n} \in \Gamma$

$$
\left\|F\left(\boldsymbol{A}^{(n)}\left(\theta_{n}\right)\right)\right\|_{L^{d}\left(X_{\gamma_{n}}^{\prime \prime}, g\right)}>c
$$

where $\boldsymbol{A}^{(n)}:=\boldsymbol{A}^{(n)}\left(\theta_{n}\right)$ is $\boldsymbol{A}\left(\theta_{n}\right)$ for the parameters $\lambda=\lambda_{n}$ and $N=N_{n}$. Using the $\Gamma$-equivariance, we can assume that $\gamma_{n}=e$ (the identity element of $\Gamma$ ). Taking a subsequence, we can also assume that $E_{n e}=E_{m e}(=: E)$ for $m \geq n \gg 1$ and $A_{n e}$ converges to $A \in M^{\prime}$ (an ASD connection on $E$ ) in the $C^{\infty}$-topology. Since $b_{n} \rightarrow 0, \boldsymbol{A}^{(n)}$ converges to $A$ up to gauge equivalence in the $\mathcal{C}^{\infty}$-topology over compact subsets in $X_{e} \backslash\left\{x_{e, s}, y_{e, s} \mid s \in S\right\}$

We have a uniform upper-bound on $\left\|F\left(\boldsymbol{A}^{(n)}\right)\right\|_{B L^{p}}$ by Proposition 4.6. Then, from $p>2$ and Corollary 8.3, there exists $\sigma>0$ such that in the Euclidean coordinates around $x_{e, s}$ and $y_{e, s}$ we have $\left|F\left(\boldsymbol{A}^{(n)}\right)\right|(x) \leq$ const $_{\sigma}$ for $\sqrt{\lambda_{n}} / 2<|x|<\sigma$ (for all $n$ ). Hence for a sufficiently small $\sigma^{\prime}<\sigma$ we have (recall: $\varepsilon=\left(c-c^{\prime}\right) / 2$ )

$$
\int_{\sqrt{\lambda_{n}} / 2<|x|<\sigma^{\prime}}\left|F\left(\boldsymbol{A}^{(n)}\right)\right|^{d} \leq \varepsilon^{d} .
$$


(Here we have used $d<+\infty$.) $\left|F\left(\boldsymbol{A}^{(n)}\right)\right|$ uniformly converges to $|F(A)|$ over $X_{e, \sigma^{\prime}}$. $\left(X_{e, \sigma}\right.$ is the complement of the balls $B\left(x_{e, s}, \sigma^{\prime}\right)$ and $B\left(y_{e, s}, \sigma^{\prime}\right)$ in $X_{e}=S_{e}^{4}$.) Hence for $n \gg 1$

$$
\left\|F\left(\boldsymbol{A}^{(n)}\right)\right\|_{L^{d}\left(X_{e, \sigma^{\prime}}, g\right)} \leq c^{\prime}+\varepsilon
$$

Therefore for $n \gg 1$

$$
\left\|F\left(\boldsymbol{A}^{(n)}\right)\right\|_{L^{d}\left(X_{e}^{\prime \prime}, g\right)} \leq c^{\prime}+\varepsilon+\varepsilon=c .
$$

This contradicts the assumption.

Proof of Theorem 2.4. Using Propositions 7.3 and 9.1, we get $(\lambda \ll 1)$

$$
\operatorname{dim}(\mathcal{M}(c, d): \Gamma) \geq \operatorname{dim}\left(\mathcal{M}\left(\operatorname{GlD}_{M^{\prime}}\right): \Gamma\right) \geq 3|S|+\operatorname{dim} H_{A_{0}}^{1} \geq 3|S|+\operatorname{dim} M_{S^{4}}(\underline{c}, d) .
$$

\section{Appendix A. Proof of the Compactness of $\mathcal{M}(c, d)$}

The purpose of this appendix is to prove the following proposition:

Proposition A.1. Let $d>2$. Let $X$ be a connected, oriented (possibly non-compact) Riemannian 4-manifold without boundary, and $E$ be a principal $S U(2)$-bundle over $X$. Let $\left\{A_{n}\right\}_{n \geq 1}$ be a sequence of $A S D$ connections on $E$ such that for any compact set $K \subset X$ we have

$$
\sup _{n \geq 1}\left\|F\left(A_{n}\right)\right\|_{L^{d}(K)}<\infty .
$$

Then there exist a subsequence (we also denote it by $\left\{A_{n}\right\}_{n \geq 1}$ ), a sequence of gauge transformations $g_{n}: E \rightarrow E$ and an $A S D$ connection $A$ on $E$ such that $g_{n}\left(A_{n}\right)$ converges to $A$ in the $\mathcal{C}^{\infty}$-topology over every compact set in $X$.

This proposition follows from Donaldson-Kronheimer [5, Proposision (4.4.9)]. But, unfortunately, I think that the proof of [5, Proposision (4.4.9)] contains a gap. I think that the proof of [5, Lemma (4.4.5)] is not correct (cf. [17, the footnote in p. 5]). The proof given below essentially uses the fact that the gauge group is $S U(2)$, and I don't know whether the same result holds for more general gauge group. In Section 2, we introduce the moduli space $\mathcal{M}(c, d)$ and state that this space is compact in the topology defined there. This compactness easily follows from the above proposition.

If $X$ is a compact manifold, then the above statement is certainly well-known (see [17, Theorem E]). So we assume that $X$ is non-compact below.

Lemma A.2. Let $Y \subset X$ be a compact submanifold with boundary in $X$ satisfying $H^{4}(X, Y ; \mathbb{Z})=0$. Let $g$ be a gauge transformation of $E$ over $Y$. (Strictly speaking, we suppose that $g$ is defined smoothly over a neighborhood of $Y$.) Then $g$ can be extended to a gauge transformation of $E$ defined over $X$. 
Proof. In this proof, we use the fact that the gauge group is $S U(2)$. Since $X$ is noncompact, $E$ is isomorphic to the product bundle $X \times S U(2)$. Hence $g$ can be identified with the map from $Y$ to $S U(2)$. $S U(2) \cong S^{3}$ is 2 -connected and $\pi_{3}(S U(2))=\mathbb{Z}$. So the obstruction on the extension of $g$ to $X$ is contained in $H^{4}(X, Y ; \mathbb{Z})$. But this is 0 . Hence $g$ can be extended over $X$.

From the second countability of $X$, there exists a sequence of compact submanifolds with boundary $Y_{k}(k \geq 1)$ such that

$$
Y_{1} \Subset Y_{2} \Subset Y_{3} \Subset \cdots, \quad X=\bigcup_{k \geq 1} Y_{k} .
$$

Lemma A.3. We can choose the above sequence so that $H^{4}\left(X, Y_{k} ; \mathbb{Z}\right)=0$ for all $k \geq 1$.

Proof. Let $X \backslash \operatorname{int}\left(Y_{k}\right)=X_{1} \sqcup X_{2} \sqcup \cdots \sqcup X_{N}$ be the decomposition into the sum of the connected components. $N$ is less than or equal to the number of the connected components of $\partial Y_{k}$. Each $X_{n}$ is a (possibly non-compact) submanifold in $X$ with non-empty boundary $\left(\partial Y_{k}=\partial X_{1} \sqcup \cdots \sqcup \partial X_{N}\right)$. We have (by the excision theorem)

$$
H^{4}\left(X, Y_{k} ; \mathbb{Z}\right)=\prod_{n=1}^{N} H^{4}\left(X_{n}, \partial X_{n} ; \mathbb{Z}\right)
$$

If $X_{n}$ is non-compact, then we get $H^{4}\left(X_{n}, \partial X_{n} ; \mathbb{Z}\right)=0$. Hence if we can arrange $Y_{k}$ so that all $X_{n}$ becomes non-compact, then we get $H^{4}\left(X, Y_{k} ; \mathbb{Z}\right)=0$. This can be easily achieved as follows: Suppose that $X_{1}, X_{2}, \cdots, X_{m}$ are compact and that $X_{m+1}, X_{m+2}$, $\cdots, X_{N}$ are non-compact. Then we set $Y_{k}^{\prime}:=Y_{k} \cup X_{1} \cup X_{2} \cup \cdots \cup X_{m}$. $Y_{k}^{\prime}$ also becomes a compact submanifold in $X$, and $X \backslash \operatorname{int}\left(Y_{k}^{\prime}\right)=X_{m+1} \cup X_{m+2} \cup \cdots \cup X_{N}$. Since each $X_{n}$ $(n \geq m+1)$ is non-compact, we get $H^{4}\left(X, Y_{k}^{\prime} ; \mathbb{Z}\right)=0$.

We suppose that the sequence $Y_{k}$ satisfies $H^{4}\left(X, Y_{k} ; \mathbb{Z}\right)=0$.

Proof of Proposition A.1. Using a collar neighborhood of $\partial Y_{k}$, we can construct a open set $U_{k} \supset Y_{k}$ (in $\left.X\right)$ which is diffeomorphic to $Y_{k} \cup_{\partial Y_{k}} \partial Y_{k} \times[0,1)$. We can arrange them so that $U_{1} \subset U_{2} \subset U_{3} \subset \cdots$. Using [17, Thoerem E'] for these $U_{k}$ and a diagonal process, we get a subsequence (we also denote it by $\left\{A_{n}\right\}$ ) satisfying the following: For each $k \geq 1$ there exist a sequence of gauge transformations $g_{n}^{(k)}$ on $U_{k}$ and an ASD connection $A^{(k)}$ defined over $U_{k}$ such that $g_{n}^{(k)}\left(A_{n}\right)$ converges to $A^{(k)}(n \rightarrow \infty)$ in the $\mathcal{C}^{\infty}$-topology over compact subsets in $U_{k}$.

For each $k \geq 1, A^{(k+1)}$ is gauge equivalent to $A^{(k)}$ over $U_{k}$. Hence there exists a gauge transformation $h_{k}$ defined over $U_{k}$ satisfying $h_{k}\left(A^{(k+1)}\right)=A^{(k)}$ on $U_{k}$. Using Lemma A.2, we have a gauge transformation $h_{k}^{\prime}$ defined over $X$ which is equal to $h_{k}$ over a neighborhood of $Y_{k}$. We have $h_{k}^{\prime}\left(A^{(k+1)}\right)=A^{(k)}$ over a neighborhood of $Y_{k}$. We define an ASD connection $A$ over $X$ by setting $A:=A^{(1)}$ over $Y_{1}$ and $A:=h_{1}^{\prime} \circ h_{2}^{\prime} \circ \cdots \circ h_{k-1}^{\prime}\left(A^{(k)}\right)$ over $Y_{k}(k \geq 2)$. This definition is well-defined. 
For each $k \geq 1$, by applying Lemma A.2 to the sequence $\left\{h_{1}^{\prime} \circ h_{2}^{\prime} \circ \cdots \circ h_{k-1}^{\prime} \circ g_{n}^{(k)}\right\}_{n \geq 1}$, we get a sequence of gauge transformations $\left\{u_{n}^{(k)}\right\}_{n \geq 1}$ defined over $X$ such that $u_{n}^{(k)}=$ $h_{1}^{\prime} \circ h_{2}^{\prime} \circ \cdots \circ h_{k-1}^{\prime} \circ g_{n}^{(k)}$ over $Y_{k}$. Then $u_{n}^{(k)}\left(A_{n}\right)$ converges to $A(n \rightarrow \infty)$ in the $\mathcal{C}^{\infty}$ topology over $Y_{k}$. In particular, there exists $n_{k} \geq 1$ satisfying

$$
\left\|A-u_{n_{k}}^{(k)}\left(A_{n_{k}}\right)\right\|_{\mathcal{C}^{k}\left(Y_{k}\right)} \leq 1 / k
$$

(Here $\|\cdot\|_{\mathcal{C}^{k}\left(Y_{k}\right)}$ is a $\mathcal{C}^{k}$-norm over $Y_{k}$ defined by using a fixed connection on $E$.) We can choose the above $n_{k}$ so that $n_{1}<n_{2}<n_{3}<\cdots$. Then $u_{n_{k}}^{(k)}\left(A_{n_{k}}\right)$ converges to $A(k \rightarrow \infty)$ in the $\mathcal{C}^{\infty}$-topology over every compact set in $X$.

\section{ApPENDix B. REVIEW OF MEAN DIMENSION}

We review the definitions and basic properties of mean dimension. For the detail, see Gromov [8], Lindenstrauss-Weiss [10] and Lindenstrauss [9].

Let $(X, d)$ be a compact metric space and $\varepsilon>0$. Let $Y$ be a topological space and $f: X \rightarrow Y$ a continuous map. We call $f$ an $\varepsilon$-embedding if we have $\operatorname{Diam} f^{-1}(y) \leq \varepsilon$ for any $y \in Y$. For example, consider $[0,1] \times[0, \varepsilon]$ with the standard Euclidean distance, and let $f:[0,1] \times[0, \varepsilon] \rightarrow[0,1]$ be the natural projection. Then $f$ is an $\varepsilon$-embedding.

We define $\operatorname{Widim}_{\varepsilon}(X, d)$ as the minimum integer $n \geq 0$ such that there exist an $n$ dimensional polyhedron $P$ and an $\varepsilon$-embedding $f: X \rightarrow P$. For example, we have $\operatorname{Widim}_{\varepsilon}([0,1] \times[0, \varepsilon]$, the Euclidean distance $)=1$ for $\varepsilon<1$. The following is one of the most basic examples (see Gromov [8, p. 332]). For its proof, see Lindenstrauss-Weiss [10, Lemma 3.2] or Tsukamoto [14, Example 4.1].

Example B.1. Consider $[0,1]^{N}$ with the $\ell^{\infty}$-distance $d_{\infty}(x, y)=\max _{i}\left|x_{i}-y_{i}\right|$. For $\varepsilon<1$ we have

$$
\operatorname{Widim}_{\varepsilon}\left([0,1]^{N}, d_{\infty}\right)=N
$$

Let $\Gamma$ be a finitely generated infinite group with a finite generating set $S . \Gamma$ is equipped with the word distance: for $\gamma, \gamma^{\prime} \in \Gamma$, we define $d\left(\gamma, \gamma^{\prime}\right)$ as the minimum integer $n \geq 0$ such that there exist $\gamma_{1}, \cdots, \gamma_{n}$ in $S \cup S^{-1}$ satisfying $\gamma^{-1} \gamma^{\prime}=\gamma_{1} \cdots \gamma_{n}$.

For a finite subset $\Omega \subset \Gamma$ and $r>0$, we define the $r$-boundary $\partial_{r} \Omega \subset \Gamma$ as the set of $\gamma \in \Gamma$ such that the $r$-ball $B(\gamma, r)$ around $\gamma$ has non-empty intersection with both $\Omega$ and $\Gamma \backslash \Omega$. Let $\Omega_{1} \subset \Omega_{2} \subset \Omega_{3} \subset \cdots$ be a sequence of finite subsets in $\Gamma$. We call this sequence an amenable sequence if for any $r>0$ we have $\left|\partial_{r} \Omega_{n}\right| /\left|\Omega_{n}\right| \rightarrow 0$ as $n$ goes to $\infty$. We call $\Gamma$ an amenable group if it has an amenable sequence. In this appendix we assume that $\Gamma$ is an amenable group with an amenable sequence $\Omega_{1} \subset \Omega_{2} \subset \Omega_{3} \subset \cdots$. For example, $\mathbb{Z}$ is an amenable group with the amenable sequence $\Omega_{n}:=\{0,1, \cdots, n\}$.

Suppose that $\Gamma$ continuously (not necessarily isometrically) acts on $X$. (We suppose that the action is a right-action.) For a finite subset $\Omega \subset \Gamma$ we define the distance $d_{\Omega}(\cdot, \cdot)$ 
by setting

$$
d_{\Omega}(x, y):=\sup _{\gamma \in \Omega} d(x \cdot \gamma, y \cdot \gamma)
$$

for $x, y \in X$. For $\varepsilon>0$ we define $\operatorname{Widim}_{\varepsilon}(X: \Gamma)$ by

$$
\operatorname{Widim}_{\varepsilon}(X: \Gamma):=\lim _{n \rightarrow \infty} \operatorname{Widim}_{\varepsilon}\left(X, d_{\Omega_{n}}\right) /\left|\Omega_{n}\right| .
$$

This limit always exists, and it is independent of the choice of amenable sequences. (see Gromov [8, pp. 336-338] and Lindenstrauss-Weiss [10, Appendix]). We define the mean dimension $\operatorname{dim}(X: \Gamma)$ by

$$
\operatorname{dim}(X: \Gamma):=\lim _{\varepsilon \rightarrow 0} \operatorname{Widim}_{\varepsilon}(X: \Gamma) .
$$

(This might be infinity.) The value of $\operatorname{dim}(X: \Gamma)$ is a topological invariant. That is, if two distances $d$ and $d^{\prime}$ on $X$ defines the same topology, then we have $\operatorname{dim}((X, d): \Gamma)=$ $\operatorname{dim}\left(\left(X, d^{\prime}\right): \Gamma\right)$. The following is the most basic result. (See Gromov [8, p. 340] and Lindenstrauss-Weiss [10, Proposition 3.1, 3.3].)

Example B.2. Let $K$ be a compact metric space and set $X:=K^{\Gamma}$. $\Gamma$ acts on $X$ by the shift action: for $x=\left(x_{\gamma}\right)_{\gamma \in \Gamma} \in X$ and $g \in \Gamma$ we set

$$
x . g=\left(y_{\gamma}\right)_{\gamma \in \Gamma}, \quad y_{\gamma}:=x_{g \gamma} .
$$

Then we have

$$
\operatorname{dim}(X: \Gamma) \leq \operatorname{dim} K
$$

where $\operatorname{dim} K$ denotes the topological covering dimension of $K$. Moreover if $K$ is a finite polyhedron, then we have

$$
\operatorname{dim}(X: \Gamma)=\operatorname{dim} K
$$

Proof. Set $N:=\operatorname{dim} K$ and we $\operatorname{suppose} \operatorname{Diam} K=1$ for $\operatorname{simplicity.~Let~} w: \Gamma \rightarrow \mathbb{R}_{>0}$ be a positive function satisfying $w(e)=1$ ( $e$ is the identity element of $\Gamma$ ) and $\sum_{\gamma \in \Gamma} w(\gamma)=2$. We define the distance $d(x, y)(x, y \in X)$ by setting

$$
d(x, y):=\sum_{\gamma \in \Gamma} w(\gamma) d\left(x_{\gamma}, y_{\gamma}\right)
$$

For $\varepsilon>0$, let $r>0$ be a positive number such that the sum of $w(\gamma)$ over $\gamma \in \Gamma \backslash B(e, r)$ is less than $\varepsilon$. Then for any $\Omega_{n}$, the natural projection

$$
\varphi: X \rightarrow K^{\Omega_{n} \cup \partial_{r} \Omega_{n}}
$$

satisfies $\operatorname{Diam}\left(\varphi^{-1}(y), d_{\Omega_{n}}\right)<\varepsilon$ for any $y \in K^{\Omega_{n} \cup \partial_{r} \Omega_{n}}$. Therefore

$$
\operatorname{Widim}_{\varepsilon}\left(X, d_{\Omega_{n}}\right) \leq N\left|\Omega_{n} \cup \partial_{r} \Omega_{n}\right| .
$$

Since $\lim _{n \rightarrow \infty}\left|\Omega_{n} \cup \partial_{r} \Omega_{n}\right| /\left|\Omega_{n}\right|=1$, we have $\operatorname{Widim}_{\varepsilon}(X: \Gamma) \leq N$. Hence $\operatorname{dim}(X: \Gamma) \leq N$. 
Next we suppose $K$ is a polyhedron. Then there exists a topological embedding $[0,1]^{N} \hookrightarrow K$. So we can assume $K=[0,1]^{N}$ with the $\ell^{\infty}$-distance. There exists a distance non-decreasing continuous map from $\left([0,1]^{N\left|\Omega_{n}\right|}, d_{\ell^{\infty}}\right)$ to $\left(X, d_{\Omega_{n}}\right)$. Then for $\varepsilon<1$

$$
\operatorname{Widim}_{\varepsilon}\left(X, d_{\Omega_{n}}\right) \geq \operatorname{Widim}_{\varepsilon}\left([0,1]^{N\left|\Omega_{n}\right|}, d_{\ell}\right)=N\left|\Omega_{n}\right| .
$$

Here we have used the result of Example B.1. Hence we get $\operatorname{Widim}_{\varepsilon}(X: \Gamma) \geq N$ for $\varepsilon<1$. Thus $\operatorname{dim}(X: \Gamma)=N$.

\title{
REFERENCES
}

[1] S. Angenent, The shadowing lemma for elliptic PDE, in Dynamics of Infinite Dimensional Systems, NATO Adv. Sci. Inst. Ser. F Comput. Systems Sci., 37, Springer, Berlin, (1987) 7-22

[2] R. Brody, Compact manifolds and hyperbolicity, Trans. Amer. Math. Soc. 235 (1978) 213-219

[3] S.K. Donaldson, An application of gauge theory to four dimensional topology, J.Differential Geom. 18 (1983) 279-315

[4] S.K. Donaldson, Connections, cohomology and the intersection forms of 4-manifolds, J.Differential Geom. 24 (1986) 275-341

[5] S.K. Donaldson, P.B. Kronheimer, The geometry of four-manifolds, Oxford University Press, New York (1990)

[6] D.S. Freed, K.K. Uhlenbeck, Instantons and four-manifolds, Second edition, Springer-Verlag, New York (1991)

[7] A. Gournay, Dimension moyenne et espaces d'applications pseudo-holomorphes, thesis, Département de Mathématiques d'Orsay (2008)

[8] M. Gromov, Topological invariants of dynamical systems and spaces of holomorphic maps: I, Math. Phys. Anal. Geom. 2 (1999) 323-415

[9] E. Lindenstrauss, Mean dimension, small entropy factors and an embedding theorem, Inst. Hautes Études Sci. Publ. Math. 89 (1999) 227-262

[10] E. Lindenstrauss, B. Weiss, Mean topological dimension, Israel J. Math. 115 (2000) 1-24

[11] M. Macrì, M. Nolasco, T. Ricciardi, Asymptotics for selfdual vortices on the torus and on the plane: a gluing technique, SIAM J. Math. Anal. 37 (2005) 1-16

[12] C.H. Taubes, Self-dual Yang-Mills connections on non-self-dual 4-manifolds, J.Differential Geom. 17 (1982) 139-170

[13] M. Tsukamoto, Gluing an infinite number of instantons, Nagoya Math. J. 188 (2007) 107-131

[14] M. Tsukamoto, Moduli space of Brody curves, energy and mean dimension, Nagoya Math. J. 192 (2008) $27-58$

[15] M. Tsukamoto, Deformation of Brody curves and mean dimension, arXiv:0706.2981, to appear in Ergodic Theory and Dynamical Systems

[16] K.K, Uhlenbeck, Connections with $L^{p}$ bounds on curvature, Commun. Math. Phys. 83 (1982) 31-42

[17] K. Wehrheim, Uhlenbeck compactness, EMS Series of Lectures in Mathematics, European Mathematical Society, Zürich (2004)

\author{
Masaki Tsukamoto \\ Department of Mathematics, Faculty of Science \\ Kyoto University
}


Kyoto 606-8502

Japan

E-mail address: tukamoto@math.kyoto-u.ac.jp 